UNIVERSIDADE DE SÃO PAULO

FACULDADE DE FILOSOFIA, CIÊNCIAS E LETRAS DE RIBEIRÃO PRETO DEPARTAMENTO DE FÍSICA E MATEMÁTICA PÓS-GRADUAÇÃO EM FÍSICA APLICADA À MEDICINA E BIOLOGIA

\title{
Simulações numéricas de Monte Carlo aplicadas no estudo das transições de fase do modelo de Ising dipolar bidimensional
}

Leandro Gutierrez Rizzi

Ribeirão Preto - SP

2009 


\section{Simulações numéricas de Monte Carlo aplicadas no estudo das transições de fase do modelo de Ising dipolar bidimensional}

Dissertação apresentada à Faculdade de Filosofia, Ciências e Letras de Ribeirão Preto da Universidade de São Paulo, como parte das exigências para a obtenção do título de Mestre em Ciências

Área de concentração:

Física Aplicada à Medicina e Biologia

Orientador:

Prof. Dr. Nelson Augusto Alves

Ribeirão Preto - SP

2009 
AUTORIZO A REPRODUÇÃO TOTAL OU PARCIAL DESTE DOCUMENTO, POR MEIO CONVENCIONAL OU ELETRÔNICO PARA FINS DE ESTUdO E PESQUISA, DESDE QUE CITADA A FONTE.

RIZZI, L. G.

Simulações numéricas de Monte Carlo aplicadas no estudo das transições de fase do modelo de Ising dipolar bidimensional / Leandro Gutierrez Rizzi; orientador Nelson Augusto Alves - Ribeirão Preto/SP, 2009.

$108 \mathrm{p}$

Dissertação (Mestrado - Programa de Pós-Graduação em Física Aplicada à Medicina e Biologia) - Departamento de Física e Matemática da Faculdade de Filosofia, Ciências e Letras de Ribeirão Preto da Universidade de São Paulo.

1. Modelo de Ising dipolar bidimensional. 2. Transições de fase. 3. Métodos de Monte Carlo. 4. Algoritmo multicanônico. 5. Algoritmo de Metropolis. 6. Somatório de Ewald. 


\section{AGRADECIMENTOS}

Primeiramente eu gostaria de agradecer ao meu pai, Antonio Carlos, pela sua sábia racionalidade que tanto influenciou a trajetória da minha vida até aqui; por toda ajuda e reconhecimento. Eu também gostaria de agradecer à minha mãe, Odette, pela bondade que tanto me influenciou, pela compreensão de ter um filho longe e pelas roupas pretas. Além de agradecer ao meu irmão Ju, que é uma pessoa que eu sempre admirei, pelo exemplo.

Em especial, gostaria de agradecer ao meu orientador, professor Nelson, pela idealização e orientação desse trabalho, além da paciência e dedicação com a qual tornou a realização desse trabalho uma tarefa menos árdua.

Ao professor Alexandre, pela atenção e incentivo dados à mim e à todos alunos da pósgraduação em Física Aplicada à Medicina e Biologia.

À professora Vanessa e ao professor Ubiraci, pela ótima didática com que conduziram as disciplinas que cursei durante o mestrado.

À "Cacá", pela "chatice", "ódio" e compreensão durante esses longos dois anos de mestrado.

Ao "Robin", pelas noites que passamos juntos no Observatório fazendo relatórios do Laboratório Avançado durante o período de graduação.

Aos amigos e colegas de laboratório, Marcelão, Ju, Brenno, Frigori, Matheus, Jacyana, Fernanda, Aquino, César, Rodrigo, Wilnice e Lindomar, pelos muitos cafés, "bandejadas", churrascos e discussões.

Aos amigos que fiz durante o mestrado: André, Khalil, Brunão, Lucas, Olavo e Denise. Em específico, ao Erbe, pelas valiosas discussões.

Ao amigo, mestre Guidolin, pelas "consultorias de cinco mil reais" gratuitas.

Ao "Bob" e ao "Furada", por trazerem de volta minha "sanidade" e pelo rock and roll. Aos meus amigos "Bart", Paulinho e Jota, por me lembrarem que eu não vivo só para a Física. Ao Diogo, pela força no início do meu mestrado, pelas centenas de conversas, as quais foram sempre muito verdadeiras, e pelos almoços de domingo.

Ao "Metralha" e ao Benette, pela distração que a nossa "banda" de Beatles nos proporcionou.

Ao Fábio, pelo suporte técnico, sem o qual não teria realizado boa parte desse trabalho.

Às secretárias do Departamento de Física e Matemática, Nilza e Sônia, e à secretária da pós-graduação, Inês, pela dedicação com que exerceram seus trabalhos.

Ao Ali, pelas aulas ministradas no CIRP, as quais foram de fundamental importância para a minha formação.

Às agências de fomento CAPES, pela bolsa de estudos, e FAPESP, pelo auxílio à pesquisa. 
Hours of lust, hours of tears passing by before my eyes today, tomorrow, yesterday... one life days of joy, day of sadness come and go to pass me by a month, a year, one hundred years, they fly K. Hansen em "March Of Time"

So I stand still In front of the crowd Excited faces What will be next? I still don't have a clue H. Kursch em "The Minstrel" 


\section{RESUMO}

RIZZI, L. G.. Simulações numéricas de Monte Carlo aplicadas no estudo das transições de fase do modelo de Ising dipolar bidimensional. Dissertação (Mestrado) - Faculdade de Filosofia, Ciências e Letras de Ribeirão Preto, Universidade de São Paulo. Ribeirão Preto, 2009.

O modelo de Ising dipolar bidimensional inclui, além da interação ferromagnética entre os primeiros vizinhos, interações de longo alcance entre os momentos de dipolo magnético dos spins. A presença da interação dipolar muda completamente o sistema, apresentando um rico diagrama de fase, cujas características têm originado inúmeros estudos na literatura. Além disso, a possibilidade de explicar fenômenos observados em filmes magnéticos ultrafinos, os quais possuem diversas aplicações em áreas tecnológicas, também motiva o estudo deste modelo. O estado fundamental ferromagnético do modelo de Ising puro é alterado para uma série de fases do tipo faixas, as quais consistem em domínios ferromagnéticos de largura $h$ com magnetizações opostas. A largura das faixas depende da razão $\delta$ das intensidades dos acoplamentos ferromagnético e dipolar. Através de simulações de Monte Carlo e técnicas de repesagem em histogramas múltiplos identificamos as temperaturas críticas de tamanho finito para as transições de fase quando $\delta=2$, o que corresponde a $h=2$. Calculamos o calor específico e a susceptibilidade do parâmetro de ordem, no intervalo de temperaturas onde as transições são observadas, para diferentes tamanhos de rede. As técnicas de repesagem permitem-nos explorar e identificar máximos distintos nessas funções da temperatura e, desse modo, estimar as temperaturas críticas de tamanho finito com grande precisão. Apresentamos evidências numéricas da existência de uma fase nemática de Ising para tamanhos grandes de rede. Em nossas simulações, observamos esta fase para tamanhos de rede a partir de $L=48$. Para verificar o quanto a interação dipolar de longo alcance afeta as estimativas físicas, nós calculamos o tempo de autocorrelação integrado nas séries temporais da energia. Inferimos daí quão severo é o critical slowing down (decaimento lento crítico) para esse sistema próximo às transições de fase termodinâmicas. Os resultados obtidos utilizando um algoritmo de atualização local foram comparados com os resultados obtidos utilizando o algoritmo multicanônico. 


\begin{abstract}
RIZZI, L. G.. Numerical Monte Carlo simulations applied to study of phase transitions in two-dimensional dipolar Ising model. Dissertation (M.Sc.) - Faculdade de Filosofia, Ciências e Letras de Ribeirão Preto, Universidade de São Paulo. Ribeirão Preto, 2009.

Two-dimensional spin model with nearest-neighbor ferromagnetic interaction and longrange dipolar interactions exhibit a rich phase diagram, whose characteristics have been exploited by several studies in the recent literature. Furthermore, the possibility of explain observed phenomena in ultrathin magnetic films, which have many technological applications, also motivates the study of this model. The presence of dipolar interaction term changes the ferromagnetic ground state expected for the pure Ising model to a series of striped phases, which consist of ferromagnetic domains of width $h$ with opposite magnetization. The width of the stripes depends on the ratio $\delta$ of the ferromagnetic and dipolar couplings. Monte Carlo simulations and reweighting multiple histograms techniques allow us to identify the finite-size critical temperatures of the phase transitions when $\delta=2$, which corresponds to $h=2$. We calculate, for different lattice sizes, the specific heat and susceptibility of the order parameter around the transition temperatures by means of reweighting techniques. This allows us to identify in these observables, as functions of temperature, the distinct maxima and thereby to estimate the finite-size critical temperatures with high precision. We present numerical evidence of the existence of a Ising nematic phase for large lattice sizes. Our results show that simulations need to be performed for lattice sizes at least as large as $L=48$ to clearly observe the Ising nematic phase. To access how the long-range dipolar interaction may affect physical estimates we also evaluate the integrated autocorrelation time in energy time series. This allows us to infer how severe is the critical slowing down for this system with long-range interaction and nearby thermodynamic phase transitions. The results obtained using a local update algorithm are compared with results obtained using the multicanonical algorithm.
\end{abstract}




\section{LISTA DE FIGURAS}

1.1 Polarização da magnetização em cada uma das direções: $x, y$ (no plano do filme) e $z$ (perpendicular ao plano do filme), em função da temperatura absoluta para filmes finos de Fe sobre $\mathrm{Cu}(100)$ com espessuras de 2,5 e 3,0 camadas atômicas (AL: "atomic layer"). Técnica utilizada: "spin-polarized secondary-electron spectroscopy". Figura adaptada de $[3] . \ldots \ldots \ldots \ldots \ldots \ldots$

1.2 Imagens obtidas utilizando a técnica SEMPA para um filme de $\mathrm{Fe}$ sobre $\mathrm{Cu}(100)$ com espessura de aproximadamente duas camadas atômicas para diferentes temperaturas: $T_{m}=281 \mathrm{~K}, T_{l}=294 \mathrm{~K}, T_{k}=296 \mathrm{~K}, T_{j}=300 \mathrm{~K}$, e $T_{i}=321 \mathrm{~K}$. A imagens são quadradas e têm largura de $44 \mu \mathrm{m}$ (exceto k e l, as quais possuem $22 \mu \mathrm{m}$ por $44 \mu \mathrm{m}$ ). Figura retirada de $[6] \ldots \ldots \ldots \ldots \ldots \ldots$

1.3 Configurações de spins do modelo de Ising dipolar bidimensional para diferentes temperaturas. Os resultados foram obtidos através de simulações de Monte Carlo. As configurações de (a) para (f) retratam o aumento da temperatura. Figura adaptada

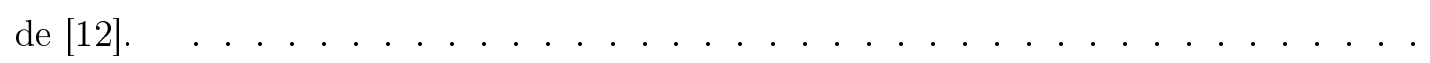

2.1 (a) Projeção da superfície $(P-V-T)$ no plano $(P-T)$ para uma substância pura; (b) projeção da superfície $(H-M-T)$ no plano $(H-T)$ para uma magneto. Figura adaptada de $[14] \ldots \ldots \ldots \ldots \ldots \ldots \ldots \ldots$

2.2 Comportamento do parâmetro de ordem $\phi$ em função da temperatura T. Na Figura (a) apresentamos o comportamento para transição de fase contínua enquanto na Figura (b) o comportamento apresentado representa uma transição de fase de primeira

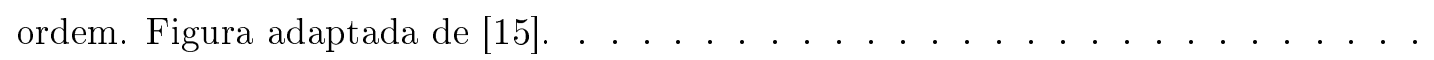

2.3 Gráficos da diferença da função de densidade de energia livre $f(T, \phi)-f_{0}(T)$ em função do parâmetro de ordem $\phi$ em diversas temperaturas. (a) Representação de transição de fase contínua. (b) Representação de transição de fase de primeira ordem.

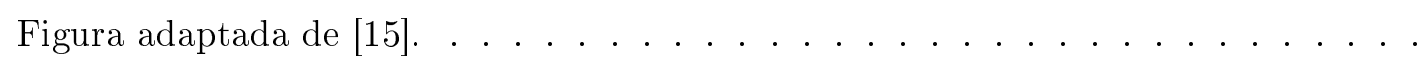


3.1 Representação da estrutura de domínio do tipo faixas de spins com largura $h=4$ para o estado fundamental. Figura retirada de [24]. . . . . . . . . . . . . . . .

3.2 Diagrama de fase obtido através de simulações numéricas de Monte Carlo. Os números de 1 a 7 representam a largura das faixas $h$ para cada uma das fases correspondentes separadas pelos círculos roxos. Figura adaptada de [21] . . . . . . . . . . . . .

3.3 Configurações de spins para a região $\delta<0,4403$. Fases do tipo tabuleiro. Figura adaptada de [32] . . . . . . . . . . . . . . . . . . . . . .

3.4 Diagrama de fase obtido via teoria de campo médio. Os números de 1 a 6 representam as regiões onde a solução mostra a presença de fases do tipo faixas com larguras correspondentes ao valor indicado. Figura retirada de [21] . . . . . . . . . . . . .

5.1 Representação das condições de contorno periódicas para interações entre primeiros vizinhos (linhas vermelhas pontilhadas) e para interações dipolares (vetores coloridos). 52

5.2 Séries temporais para a energia por spin $\langle E / N\rangle$ geradas pelo algoritmo de Metropolis para $L=56$ em diferentes temperaturas. . . . . . . . . . . . . . . .

5.3 Séries temporais para o parâmetro de ordem $O_{h v}$ (sem módulo) geradas pelo algoritmo de Metropolis para $L=56$ em diferentes temperaturas. . . . . . . . . . . . .

5.4 Histogramas das séries produzidas para a energia por spin $E / N$ e parâmetro de ordem $O_{h v}$ para $L=56$ em diferentes temperaturas. (a) e (b) correspondem a transição de fase nemática-tetragonal e (c) e (d) à transição de fase faixas-nemática. . . . . . . . .

5.5 Calor específico $C_{v}$ e energia média por spin $\langle E / N\rangle$ em função da temperatura $T$ para $L=16,32,48$ nas figuras (a) e (b), e $L=56,72$ em (c) e (d). Dentro da Fig. (a): $C_{v}$ com estimativas de barras de erro para $L=48 \ldots \ldots \ldots \ldots$

5.6 Configurações típicas de spins para $L=56$ em temperaturas nas fases: (a) faixas, (b) nemática e (c) tetragonal. A configuração na fase do tipo faixas representa $E / N=$ $-1,2028, O_{h v}=0,9873$; na fase nemática, $E / N=-1,1172, O_{h v}=0,7903$; e na fase líquido tetragonal $E / N=-1,0201$ e $O_{h v}=0,1777 \ldots \ldots \ldots \ldots$

5.7 Configurações de spins nas temperaturas de transição para $L=56$. A configuração apresentada em (a) tem $E / N=-1,1670, O_{h v}=0,9205$; enquanto que em (b), $E / N=-1,0769$ e $O_{h v}=0,5633 \ldots \ldots \ldots \ldots \ldots \ldots$

5.8 Susceptibilidade e parâmetro de ordem para diversos tamanhos de rede em função da

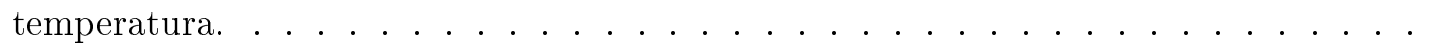

5.9 Tempo de autocorrelação integrado $2 \tau_{\text {int }}$ para tamanhos de rede $L=16$ (a), $L=32$ (b), $L=48$ (c) e $L=56$ (d) em função do tamanho das séries temporais $2^{k}$. . . . 66

5.10 Série de Monte Carlo obtida por meio do algoritmo multicanônico para $L=16$. . . 
5.11 Parâmetros multicanônicos $a(E)$ em função da energia por spin $E / N$ para diferentes tamanhos de rede $L \ldots \ldots \ldots$. . . . . . . . . . . . . . . . . . . . . . . 68

5.12 Parâmetros multicanônicos $b(E)$ em função da energia por spin $E / N$ para diferentes tamanhos de rede $L \ldots \ldots \ldots \ldots$

5.13 Histogramas para as séries produzidas utilizando o algoritmo multicanônico para diferentes tamanhos de rede. . . . . . . . . . . . . . . . . . . . . . . 69

5.14 Histogramas para as séries produzidas em diferentes estágios da atualização $(20-140$ atualizações) dos parâmetros $a(E)$ e $b(E)$ para a rede de tamanho $L=48 . \quad$. . . . . 70

5.15 Grandezas termodinâmicas em função da temperatura $T$ resultantes da repesagem das séries obtidas pelo algoritmo multicanônico para redes de tamanho $L=16,32$ e 48 . 71

5.16 Probabilidade canônica de Gibbs na temperatura crítica de tamanho finito $T_{c}^{(2)}=$ 0,8131 , para $L=48$, calculada através da equação (4.3.8) a partir dos parâmetros multicanônicos $a(E)$ e $b(E)$. . . . . . . . . . . . . . . . . . . 73

A.1 Gráficos de $f_{p}(\alpha r)$ para diferentes valores de $\alpha$ e $p, \operatorname{com} \alpha=5 / L \ldots \ldots$. . . . . 80

A.2 Representação do modo convencional de efetuar os somatórios infinitos sobre as componentes do vetor $\vec{n}$ na expressão (A.3.1). Figura extraída de [48]. . . . . . . . . . . 


\section{LISTA DE TABELAS}

3.1 Resultados de cálculos analíticos para as regiões de equilíbrio em $\delta$ para a largura $h$ das faixas. Dados retirados de [32] . . . . . . . . . . . . . . 31

3.2 Regiões de estabilidade para as fases do tipo $\langle 1 . n\rangle$. Dados retirados de [32] . . . . . 35

5.1 Temperaturas $T_{0}$ para produções das séries temporais a partir do algoritmo de Metropolis e utilizadas para repesagem. . . . . . . . . . . . . . . . .

$5.2 T_{c}^{(i)}$ : temperaturas críticas de tamanho finito para as transições de fase observadas para tamanhos de rede $L$ definidas pelo máximo do calor específico $\left.C_{v}^{(i)}\right|_{\max }$ como mostrado nas Fig. 5.5(a) e 5.5(b) . . . . . . . . . . . . . . . . . .

5.3 Máximos da susceptibilidade e as correspondentes temperaturas críticas $T_{c}^{(i)}$ identificadas na Fig. 5.8.

5.4 Temperaturas críticas de tamanho finito para as transições de fase observadas para tamanhos de rede $L$ definidas pelo máximo de calor específico $\left.C_{v}\right|_{\max }$ obtidos através do algoritmo multicanônico, como mostrado nas Figura 5.15(a) . . . . . . . . . . . .

5.5 Temperaturas críticas de tamanho finito para as transições de fase observadas para tamanhos de rede $L$ definidas pelo máximo da susceptibilidade do parâmetro de ordem $\chi\left(O_{h v}\right)_{\max }^{(i)}$ obtidos através do algoritmo multicanônico, como mostrado nas Figura

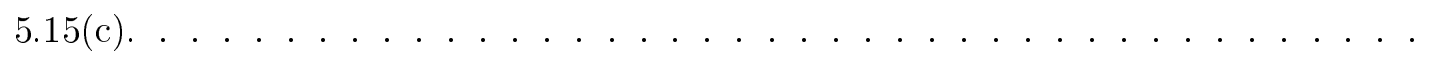

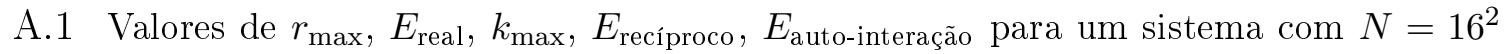
spins em função do parâmetro $\alpha$ para $\sigma_{i}=1(i=1, \ldots, N)$. . . . . . . . . . 91

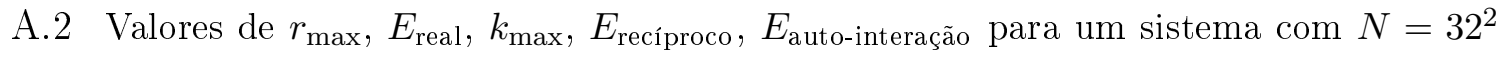
spins em função do parâmetro $\alpha$ para $\sigma_{i}=1(i=1, \ldots, N)$. . . . . . . . . . . 91

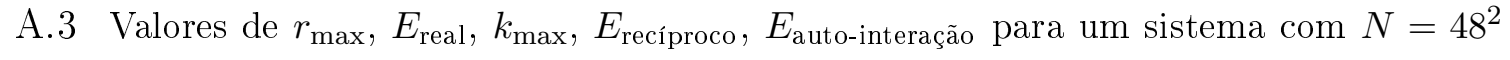
spins em função do parâmetro $\alpha$ para $\sigma_{i}=1(i=1, \ldots, N)$. . . . . . . . .

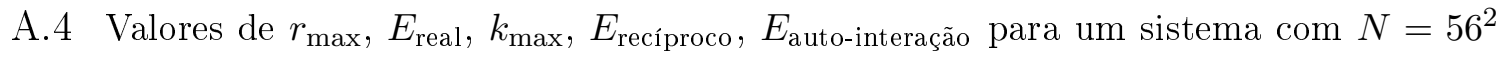
spins em função do parâmetro $\alpha$ para $\sigma_{i}=1(i=1, \ldots, N)$. . . . . . . . . . 
A.5 Valores de $r_{\max }, E_{\text {real }}, k_{\max }, E_{\text {recíproco }}, E_{\text {auto-interação para um sistema com } N=72^{2}}$ spins em função do parâmetro $\alpha$ para $\sigma_{i}=1(i=1, \ldots, N)$. . . . . . . . . . . 92

B.1 Tempo necessário, em segundos, para cada um dos tamanhos de rede $L$, para realizar um único passo de Monte Carlo, ou seja, para realizar $L^{2}$ tentativas de atualização dos spins. . . . . . . . . . . . . . . . . . . . . . . . 100 


\section{Sumário}

1 Introdução 14

2 Transições de fase $\quad 18$

2.1 Introdução . . . . . . . . . . . . . . . . . . . 18

2.2 Classificação básica e universalidade . . . . . . . . . . . . . . . . 20

2.3 Teoria de Landau e sistemas de tamanho finito . . . . . . . . . . . . . . . . . 22

3 O modelo de Ising dipolar bidimensional $\quad 27$

3.1 Descrição do modelo . . . . . . . . . . . . . . . . . . . . . 27

3.2 Diagrama de fase . . . . . . . . . . . . . . . . . . 30

4 Métodos de Monte Carlo $\quad 37$

4.1 Conceitos em Mecânica Estatística . . . . . . . . . . . . . . . . 37

4.2 Cadeias de Markov . . . . . . . . . . . . . . . . . . . . . 41

4.2 .1 Algoritmo de Metropolis . . . . . . . . . . . . . . 43

4.2 .2 Tempo de autocorrelação integrado . . . . . . . . . . . . . . . 44

4.3 Algoritmo multicanônico . . . . . . . . . . . . . . . . . . 45

5 Simulação do modelo de Ising dipolar bidimensional 49

5.1 Condições de contorno periódicas . . . . . . . . . . . . . . 5 51

5.2 Metodologias para análise de dados . . . . . . . . . . . . . . 54

5.2 .1 Técnica de repesagem . . . . . . . . . . . . . 54

5.2 .2 O método de Jackknife . . . . . . . . . . . . . . . . 56

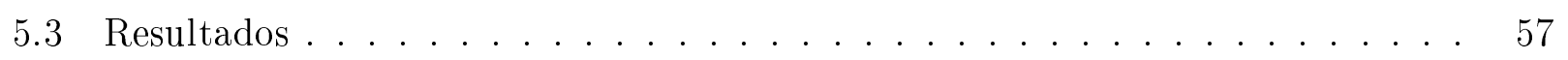

5.3 .1 Algoritmo de Metropolis . . . . . . . . . . . . . . 57

5.3 .2 Algoritmo multicanônico . . . . . . . . . . . . . 67

6 Conclusões $\quad 75$ 
$\begin{array}{ll}\text { A O somatório de Ewald em redes bidimensionais } & 78\end{array}$

A.1 Aspectos analíticos do método de Ewald . . . . . . . . . . . . . . 78

A.2 Casos particulares . . . . . . . . . . . . . . . . . . . 83

A.2.1 Interação de Coulomb . . . . . . . . . . . . . . . . . 83

A.2.2 Interação dipolar . . . . . . . . . . . . . . . . . . . . . . . 84

A.3 Método de armazenamento . . . . . . . . . . . . . . . . . . 85

A.4 Estudo numérico da convergência dos somatórios . . . . . . . . . . . . . . . . . 90

A.5 Demonstrações das relações auxiliares . . . . . . . . . . . . . . . . . . . . 92

A.5.1 Demonstração da relação $\S 1$. . . . . . . . . . . . . . . . . . . . 92

A.5.2 Demonstração da relação $\S 2 \ldots \ldots$. . . . . . . . . . . . . 94

A.5.3 Demonstração da relação $\S 3 \ldots \ldots$. . . . . . . . . . . . 96

$\begin{array}{ll}\text { B Informações técnicas } & 100\end{array}$

Referências Bibliográficas . . . . . . . . . . . . . . . . 102 


\section{Capítulo 1}

\section{Introdução}

Filmes finos ou materiais quase bidimensionais ${ }^{1}$ têm muitas aplicações tecnológicas. No caso de filmes metálicos sobre substratos metálicos, encontramos usos em eletrônica, armazenamento de dados e catálises de reações. Filmes moleculares são, por sua vez, bastante utilizados em biotecnologia e farmacologia. Com os avanços nas técnicas de crescimento de filmes, tais como a epitaxia de feixe atômico (ou molecular) [1], e nos métodos de caracterização desses filmes, tais como o efeito Kerr magneto-óptico de superfície [2], inúmeros estudos experimentais têm sido realizados nos últimos anos. Tais estudos não são motivados somente pela importância tecnológica desses sistemas, entender como certas propriedades magnéticas macroscópicas surgem nesses materiais é também um desafio para o nosso conhecimento sobre as interações atômicas.

Atualmente, um conhecimento detalhado das interações microscópicas faz-se essencial para o desenvolvimento de materiais com características específicas para determinada aplicação tecnológica. São frequentes as preocupações sobre como essas interações são afetadas por fatores tais como composição e preparação, e como esses fatores determinam as propriedades dos materiais. Por exemplo, o uso de filmes magnéticos ultrafinos para armazenamento de dados requer que a magnetização do filme seja escolhida e lida com graus elevados de precisão e resolução espacial. Os parâmetros de operação de tais dispositivos geralmente são pré-determinados ou restritos, assim, a habilidade de projetar o material para otimizar sua performance, conhecendo suas restrições, pode trazer um benefício tecnológico significativo.

Dentre os filmes mais estudados estão: i) filmes metálicos ultrafinos (i.e., com poucas camadas atômicas) em substratos metálicos tais como Fe sobre $\mathrm{Cu}(100)$ e Co sobre $\mathrm{Au}(111)$; e ii) filmes de camadas de terras-raras, os quais ocorrem nas estruturas perovskita do tipo (TR) $\mathrm{Ba}_{2} \mathrm{Cu}_{3} \mathrm{O}_{7-\nu}$, onde TR representa uma terra-rara da série dos lantanídeos (i.e., $\mathrm{Nd}^{3+}$,

\footnotetext{
${ }^{1} \mathrm{O}$ termo "quase" é empregado aqui no sentido de que os sistemas em questão são constituidos de apenas uma ou poucas camadas atômicas.
} 


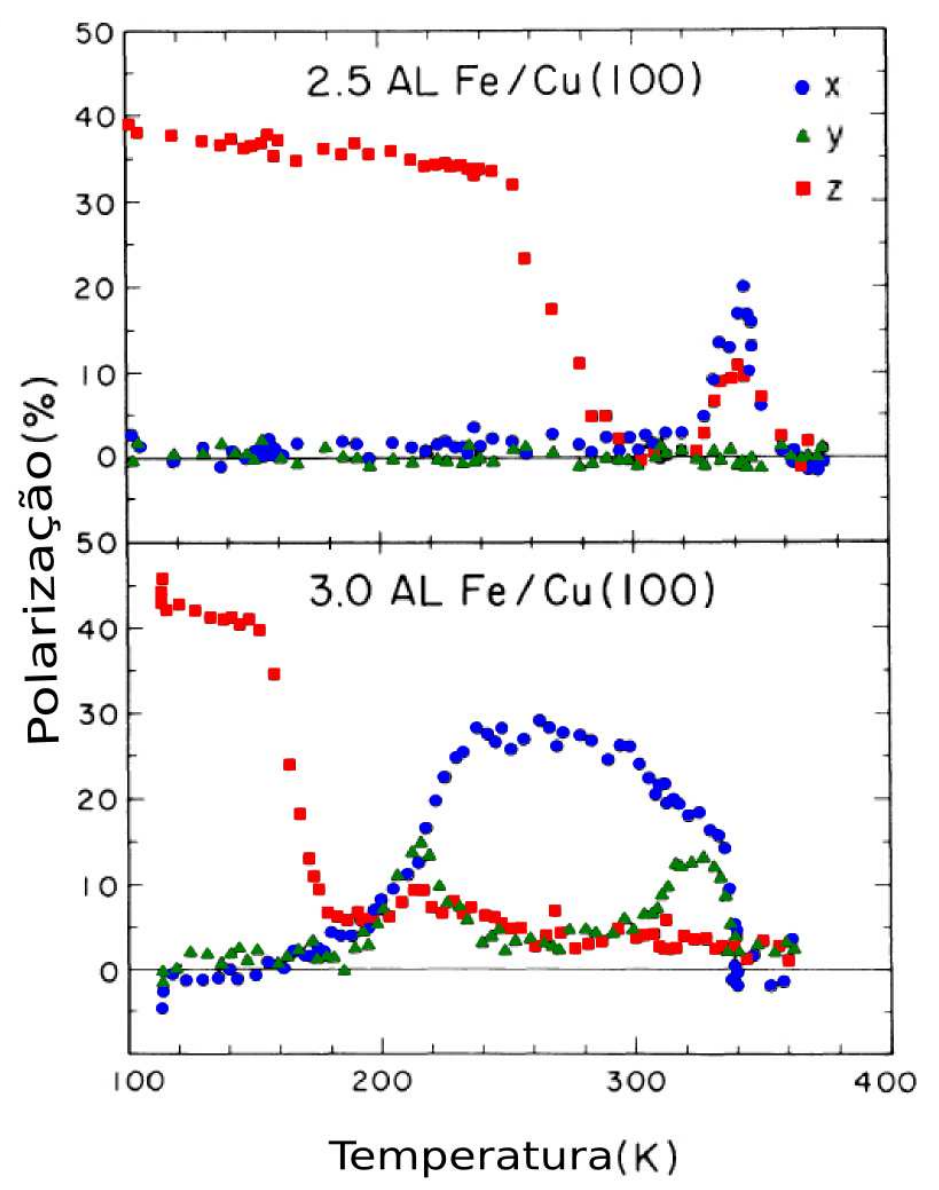

Figura 1.1: Polarização da magnetização em cada uma das direções: $x, y$ (no plano do filme) e $z$ (perpendicular ao plano do filme), em função da temperatura absoluta para filmes finos de $\mathrm{Fe}$ sobre $\mathrm{Cu}(100)$ com espessuras de 2,5 e 3,0 camadas atômicas (AL: "atomic layer"). Técnica utilizada: "spin-polarized secondary-electron spectroscopy". Figura adaptada de [3].

$\mathrm{Er}^{3+}, \mathrm{Y}^{3+}, \mathrm{Gd}^{3+}$, etc). Tanto estudos experimentais quanto teóricos têm revelado propriedades magnéticas não usuais nesses sistemas. Como mostrado na Figura 1.1, para filmes suficientemente finos de $\mathrm{Fe}$ sobre substratos de $\mathrm{Cu}$, a polarização da magnetização alinha-se numa orientação perpendicular ao plano do filme a partir de uma temperatura característica [3]. Esta temperatura é denominada temperatura de transição de reorientação. Diminuindose a espessura do filme de 3,0 para 2,5 monocamadas atômicas nota-se um valor maior para essa temperatura. Outros estudos experimentais $[4,5]$ mostram que uma característica de tal ordenamento fora do plano é a formação de padrões caracterizados por domínios de grupos ferromagnéticos de spins ordenados. Estudos recentes [6] mostram que esses padrões são contituidos por faixas alternadas ferromagnéticas ou na forma de labirinto (vide Figura 1.2). Nesta figura, as faixas escuras indicam um dado sentido dos spins, enquanto que as faixas claras indicam spins no sentido contrário. Para uma temperatura igual a $321 \mathrm{~K}$ temos uma 

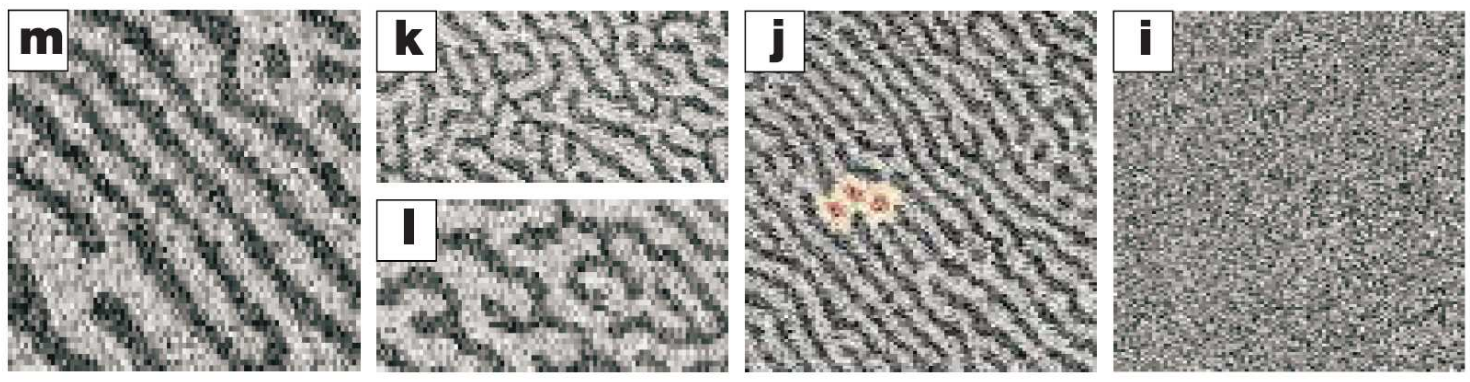

Figura 1.2: Imagens obtidas utilizando a técnica SEMPA para um filme de Fe sobre $\mathrm{Cu}(100)$ com espessura de aproximadamente duas camadas atômicas para diferentes temperaturas: $T_{m}=281 \mathrm{~K}$, $T_{l}=294 \mathrm{~K}, T_{k}=296 \mathrm{~K}, T_{j}=300 \mathrm{~K}$, e $T_{i}=321 \mathrm{~K}$. A imagens são quadradas e têm largura de $44 \mu \mathrm{m}$ (exceto $\mathrm{k} \mathrm{e} 1$, as quais possuem $22 \mu \mathrm{m}$ por $44 \mu \mathrm{m}$ ). Figura retirada de [6].

fase paramagnética (desordenada). Conforme diminuimos a temperatura, encontramos uma fase relativamente ordenada, caracterizada por faixas e bolhas. Posteriormente passamos para uma fase do tipo labirinto até chegar, finalmente, à fase do tipo faixas a $294 \mathrm{~K}$.

Com o intuito de entender a formação de estados ordenados em sistemas magnéticos quase bidimensionais, incluindo o padrão de labirintos e de faixas, recorrer a modelos de sistemas magnéticos simples pode ser uma boa estratégia. Estudos teóricos sobre sistemas magnéticos geralmente evitam incluir a interação dipolar, utilizando o argumento de que a contribuição da interação dipolar é pequena comparada com a contribuição da interação de troca ferromagnética. Para sistemas quase bidimensionais, no entanto, a interação dipolar contribui de maneira significativa. De fato, uma descrição teórica realística desses sistemas deve incluir a interação de troca, a interação dipolar e a anisotropia magnetocristalina da rede, pois o ordenamento magnético de longo alcance em sistemas quase bidimensionais requer a quebra da simetria através da anisotropia magnética [7]. Em particular, por causa da anisotropia magnética e da espessura dos filmes, muitos autores procuraram realizar esta discussão teórica por meio do modelo de Ising bidimensional dipolar. Este modelo inclui, além da interação de troca ferromagnética usual do modelo de Ising puro [8, 9], interações entre os momentos de dipolo magnético dos spins. A interação dipolar, ao contrário da interação de troca, possui um carácter antiferromagnético, o que leva à competição entre as interações. Essa competição entre os dois tipos de interação atribui ao modelo algumas características que também estão presentes nos filmes magnéticos ultrafinos. Em particular, apresenta a formação de padrões caracterizados por domínios ferromagnéticos. O modelo de Ising dipolar bidimensional, assim como o modelo de Heisenberg bidimensional, encontra-se dentro de uma classe muito mais geral de modelos. São modelos que consideram interações competitivas e que apresentam 
(6)

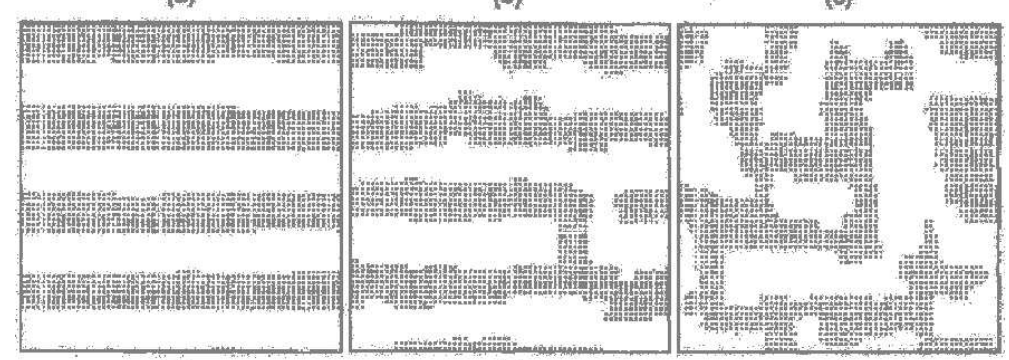

(d)

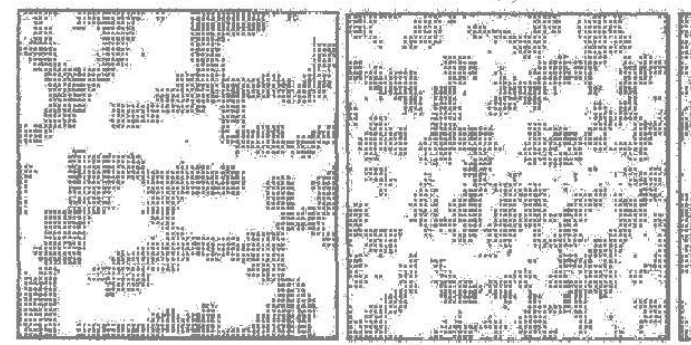

(n)

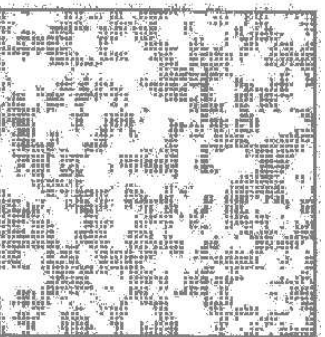

Figura 1.3: Configurações de spins do modelo de Ising dipolar bidimensional para diferentes temperaturas. Os resultados foram obtidos através de simulações de Monte Carlo. As configurações de (a) para (f) retratam o aumento da temperatura. Figura adaptada de [12].

fases espacialmente não homogêneas (e.g., tipo faixas, bolhas, lamelas ou ainda tabuleiro de xadrez), associadas com quebras das simetrias translacionais e/ou rotacionais [10, 11].

Como na maioria dos modelos, a presença de certos termos, como o termo de interação entre os momentos de dipolo dos spins no modelo de Ising dipolar, pode trazer grandes dificuldades, tanto teóricas quanto computacionais. Contudo, principalmente graças ao avanço da tecnologia na área computacional, as ferramentas da Mecânica Estatística vem mostrando-se muito poderosas nesses tipos de estudo. Simulações numéricas são bastante utilizadas para determinar o comportamento de vários modelos simplificados, mas não menos interessantes, que apresentam transições de fase e não são exatamente solúveis. Por exemplo, na Figura 1.3, apresentamos configurações de spins para o modelo de Ising dipolar bidimensional retiradas de resultados obtidos por meio de simulações de Monte Carlo [12]. Esses e outros resultados mostram que as simulações de Monte Carlo, embora inerentemente limitadas a sistemas muito menores que os sistemas experimentais, podem fornecer informações úteis a respeito do sistema. Em particular, informações sobre como as estruturas de domínios são afetadas pelo valor de um dado parâmetro assim como sua dependência com a temperatura. Apresentamos nessa dissertação um estudo sobre o modelo de Ising dipolar bidimensional dando enfoque nas simulações numéricas de Monte Carlo. Como objetivos principais consideramos a identificação das fases e também a determinação da natureza de cada uma das transições de fase termodinâmicas. 


\section{Capítulo 2}

\section{Transições de fase}

Transições de fase e fenômenos críticos ocorrem numa enorme variedade de sistemas e situações de interesse físico. Como exemplos de sistemas físicos temos: fluidos simples (água, $\mathrm{CO}_{2}$, etc) ou com vários componentes, magnetos, ligas metálicas e compostos iônicos, ferroelétricos, cristais líquidos, polímeros, superfluidos, supercondutores, etc. As transições de fase podem ser de diversos tipos. Como exemplos, temos transições no equilíbrio, transições dinâmicas, transições em sistemas desordenados ("vidros de spin" e vidros reais), transições geométricas, transições quânticas, etc [13].

Apresentamos a seguir uma pequena introdução às transições de fase. Abordamos primeiramente a classificação termodinâmica básica que envolve a natureza das transições. Introduzimos o conceito de parâmetro de ordem e também alguns conceitos envolvidos na teoria fenomenológica de Landau. A partir da teoria de Landau fazemos considerações sobre sistemas de tamanho finito, os quais são invariavelmente empregados em simulações numéricas. Por fim, discutimos um método numérico para determinação da ordem da transição.

\subsection{Introdução}

Embora existam transições de fase - como a condensação da água, o derretimento do gelo, etc - com as quais estamos bastante familiarizados, alguns de seus aspectos físicos básicos ainda não são completamente entendidos. Do ponto de vista da Mecânica Estatística, uma conexão é estabelecida entre as fases macroscópicas do sistema e suas propriedades microscópicas (tais como as forças entre os átomos, etc). No entanto, ainda que a interação efetiva entre os átomos seja conhecida (por exemplo, do tipo van der Waals, semelhante ao potencial de LennardJones), é difícil predizer teoricamente em quais condições termodinâmicas (temperatura $T$, pressão $P$, etc) as transições de fase irão ocorrer. Métodos analíticos para a previsão de diagramas de fase são úteis sobretudo para problemas "na rede": por exemplo, transições de 
fase ordem-desordem em ligas de $\mathrm{Cu}_{3} \mathrm{Au}$ (cúbicas de face centrada). Quando na fase ordenada, os átomos de Au povoam principalmente uma das quatro subredes cúbicas simples, enquanto na fase desordenada, os átomos de $\mathrm{Au}$ ficam igualmente distribuídos por todas as quatro subredes. Para problemas "fora da rede", tais como as transições sólido-líquido e líquido-gás do Ar, temos que confiar inteiramente em abordagens de simulações numéricas.

Quando nos referimos a transições de fase pensamos também na sua natureza, ou seja, na sua ordem. A classificação termodinâmica básica considera um potencial termodinâmico (por exemplo, a energia livre Helmholtz $F$ ) e suas derivadas na transição. Se a primeira derivada de $F$ exibe singularidades do tipo salto, a transição é chamada de primeira ordem, se a primeira derivada for contínua, mas a segunda derivada for singular, temos uma transição de segunda ordem (ou contínua), e esse é um ponto crítico no diagrama de fase.

Pontos críticos são muito mais especiais do que transições de primeira ordem. Por exemplo, na transição gás-líquido de um sistema composto de uma substância pura, as energias livres de Gibbs $G_{\text {gás }}(T, P)$ e $G_{\text {líquido }}(T, P)$ das duas fases devem ser iguais, o que significa que a temperatura no plano da pressão da transição gás-líquido revela-se como uma linha de transição $T_{\text {condensação }}(P)$ (vide Figura 2.1(a)). A condição adicional de que a compressibilidade diverge é satisfeita apenas em um único ponto, o ponto crítico $T_{c}=T_{\text {condensação }}\left(P_{c}\right)$, onde a linha de transição gás-líquido termina. Também podem ocorrer fenômenos multicríticos, os quais correspondem à existência de pontos multicríticos. São pontos caracterizados pelo encontro de linhas críticas distintas e ocorrem geralmente em diagramas de fase de sistemas mais complexos (por exemplo, misturas de dois fluidos).

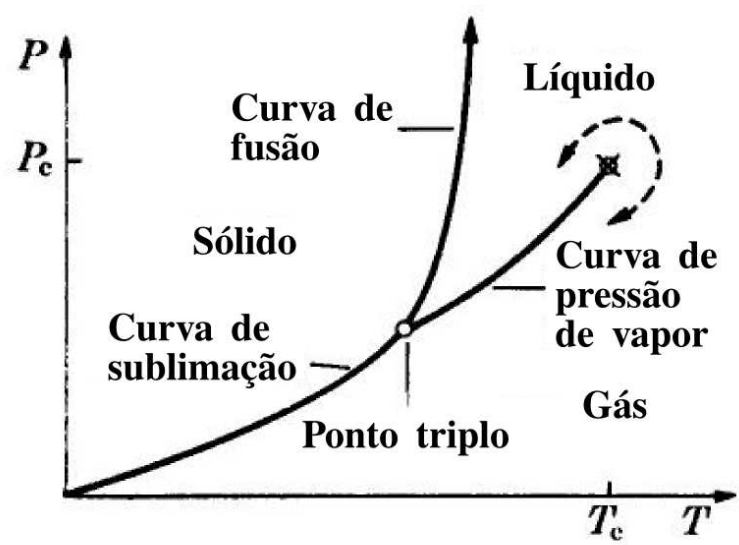

(a) Fluído

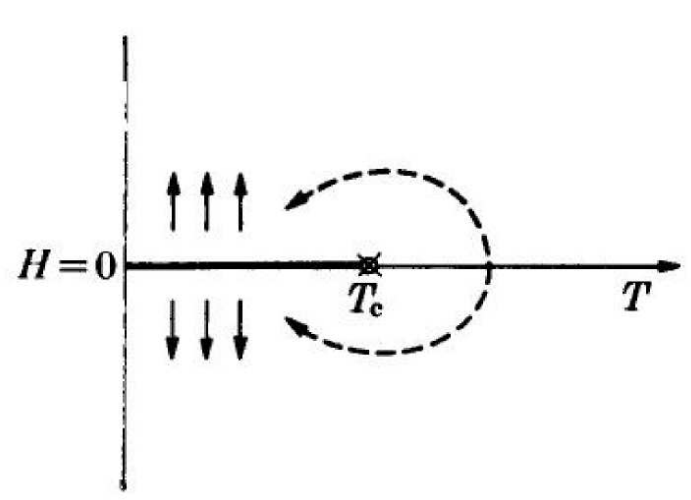

(b) Magneto

Figura 2.1: (a) Projeção da superfície $(P-V-T)$ no plano $(P-T)$ para uma substância pura; (b) projeção da superfície $(H-M-T)$ no plano $(H-T)$ para uma magneto. Figura adaptada de [14]. 


\subsection{Classificação básica e universalidade}

Iniciamos essa seção introduzindo o conceito de parâmetro ordem. Além de ser útil defini-lo dentro do contexto da classificação das transições de fase, o parâmetro de ordem é fundamental para o entendimento da teoria de Landau, a qual é apresentada na próxima seção.

Geralmente definimos o parâmetro de ordem como uma variável termodinâmica intensiva, a qual permite distiguir as fases do sistema. Como exemplos de parâmetro de ordem temos a diferença de densidades $\Delta \rho=\rho_{\text {líquido }}-\rho_{\text {gás }}$, no caso de uma substância pura, e a magnetização por spin $m$, no caso de um magneto. Usualmente o parâmetro de ordem é escolhido de tal maneira que seja igual a zero na fase desordenada e diferente de zero na outra fase, a fase ordenada. Considerando o potencial termodinâmico $F$, que tem o "campo" $H$ conjugado ao parâmetro de ordem $\phi$ como uma "variável natural" (além da temperatura $T$ ), defini-se a derivada primeira de $F$ em relação a $H$ como o parâmetro de ordem:

$$
\phi=-\left(\frac{\partial F}{\partial H}\right)_{T} .
$$

A outra derivada de $F$ é a entropia $S=(\partial F / \partial T)_{H}$. Podemos observar a mudança de $\phi$ quando uma variável independente varia, por exemplo a temperatura $T$. Se $\phi$ anula-se após a transição de maneira contínua, temos uma transição de segunda ordem (vide Figura 2.2(a)); ou, se $\phi$ anula-se de maneira descontínua, temos uma transição de primeira ordem (vide Figura 2.2(b)). Em uma transição de segunda ordem, a susceptibilidade $\chi$ do parâmetro de ordem e o calor específico $c_{H}$ têm tipicamente singularidades tipo lei de potência,

$$
\begin{gathered}
\chi \equiv-\left(\frac{\partial^{2} F}{\partial H^{2}}\right)_{T} \propto\left|1-\frac{T}{T_{c}}\right|^{-\gamma}, \\
c_{H} \equiv-T\left(\frac{\partial^{2} F}{\partial T^{2}}\right)_{H} \propto\left|1-\frac{T}{T_{c}}\right|^{-\alpha},
\end{gathered}
$$

assim como o parâmetro de ordem,

$$
\phi \propto\left|1-\frac{T}{T_{c}}\right|^{\beta} .
$$

Os expoentes $\gamma, \alpha$ e $\beta$ são conhecidos como expoentes críticos [14].

Para transições de primeira ordem, geralmente uma descontinuidade $\Delta S$ ocorre na entropia, correspondendo ao calor latente $\Delta Q=T_{c} \Delta S$. Neste caso, a segunda derivada de $F$ é finita em $T$, e também diverge nos limites de estabilidade termodinâmica em $T_{0}$ e $T_{1}$ das fases desordenada e ordenada respectivamente [15]. 

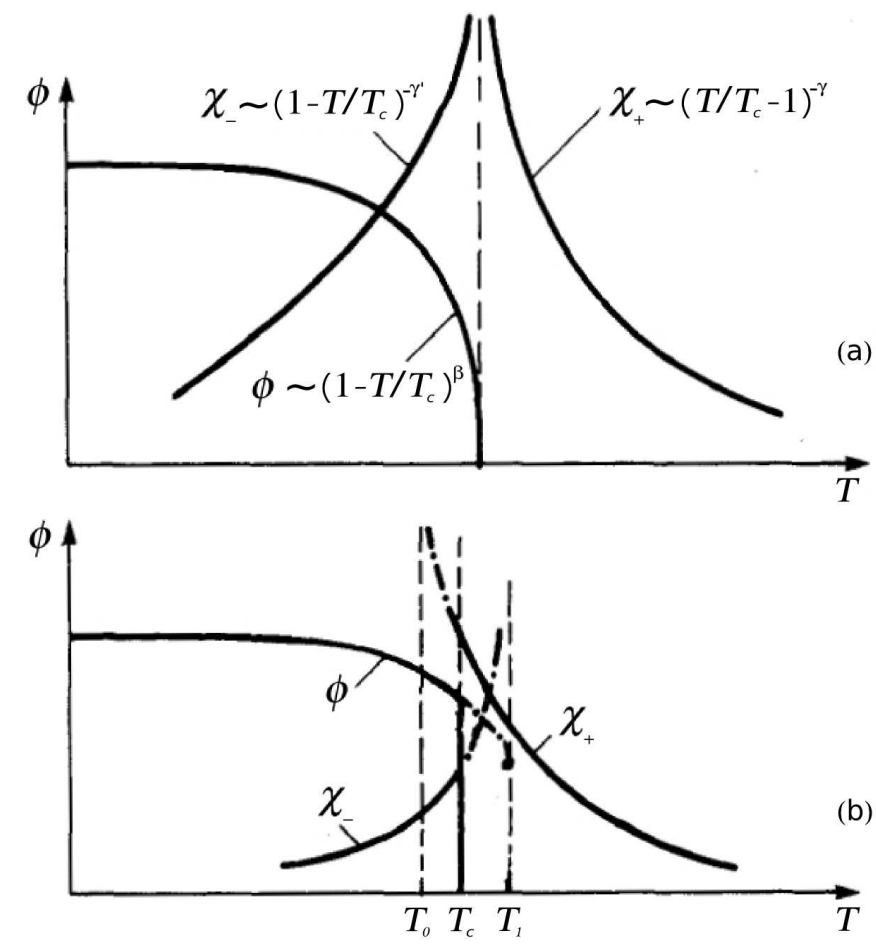

Figura 2.2: Comportamento do parâmetro de ordem $\phi$ em função da temperatura $T$. Na Figura (a) apresentamos o comportamento para transição de fase contínua enquanto na Figura (b) o comportamento apresentado representa uma transição de fase de primeira ordem. Figura adaptada de [15].

Sempre que temos uma transição contínua, temos também um fenômeno crítico associado. Os fenômenos críticos surgem devido a uma divergência no comprimento de correlação

$$
\xi \sim\left|1-\frac{T}{T_{c}}\right|^{-\nu}
$$

o qual está associado às flutuações do parâmetro de ordem. Aqui introduzimos o expoente $\nu$, o qual também faz parte do conjunto de expoentes críticos [14]. Perto do ponto crítico, o comprimento de correlação é muito grande e somente as estruturas de configurações do sistema do tamanho dessa escala de comprimento são importantes, enquanto que o comportamento do sistema em uma escala muito menor torna-se irrelevante ${ }^{1}$. Esse fato é responsável pelo comportamento de escala das funções termodinâmicas próximo a um ponto crítico e também constitui a base para a abordagem via grupo de renormalização ${ }^{2}$. Esse comportamento de escala, a qual permite a renormalização, junto com a universalidade, são as principais carac-

\footnotetext{
${ }^{1}$ Essa é conhecida como a hipótese de escala.

${ }^{2}$ Aqui estamos interessados apenas em apresentar uma introdução básica sobre transições de fase. A abordagem do grupo de renormalização em si não faz parte do escopo dessa dissertação. Para mais informações vide Ref. [16].
} 
terísticas observadas nos fenômenos críticos. A universalidade é definida pelo conjunto de expoentes críticos iguais associados à diversas transições contínuas. Um dado conjunto de expoentes indica um comportamento universal mesmo para sistemas bastante diferentes. Por exemplo, o modelo do gás de van der Waals está na mesma classe de universalidade que a teoria de campo médio.

Em uma transição de primeira ordem, no entanto, não há comprimento de correlação divergente, e em geral não se pode restringir a atenção para fenômenos que ocorrem em uma escala característica. Assim, nenhuma universalidade como nos fenômenos críticos é esperada. De qualquer maneira, a teoria do grupo de renormalização também tem contribuído para uma melhor compreensão das transições de primeira ordem. Transições de primeira ordem exibem um comportamento de escala de tamanho finito semelhante ao comportamento de escala em transições contínuas. As transições de primeira ordem aparecem no âmbito da teoria do grupo de renormalização através da descontinuidade dos pontos fixos. Assim, a teoria do grupo de renormalização ajuda-nos a entendê-las, embora não haja comprimentos divergentes.

A caracterização da ordem das transições de fase termodinâmicas pode ser feita através da análise de escala de tamanho finito (no inglês "finite-size scaling"). Neste contexto, o máximo do calor específico é descrito pela relação

$$
\left.C_{v}\right|_{\max } \propto N^{\alpha / d \nu}
$$

Para sistemas "na rede", $N$ corresponde ao volume do sistema (ou número de partículas) e é dado por $L^{d}$, onde $L$ é a dimensão linear do sistema e $d$ é a dimensão espacial. Temos também uma relação de escala de tamanho finito para o máximo da susceptibilidade, a qual é dada por

$$
\chi_{\max } \propto N^{\gamma / d \nu}
$$

O caráter de uma transição de fase de primeira ordem está relacionado com expoente crítico $d \nu=1[17,18]$. Da relação de hiperescala $\alpha=2-d \nu$, e assumindo uma transição de primeira ordem, obtém-se $\alpha / d \nu=1$ e $\gamma / d \nu=1$.

\subsection{Teoria de Landau e sistemas de tamanho finito}

A teoria de Landau serve como ponto de partida para uma discussão sobre efeitos de tamanho finito nas transições de fase. A idéia central da teoria de Landau é bastante simples e consiste em expandir a densidade de energia livre em função das potências do parâmetro de ordem $\phi$ :

$$
f(T, \phi) \equiv f_{0}(T)+A(T) \phi^{2}+B(T) \phi^{4}+\ldots
$$


Os coeficientes $f_{0}(T), A(T)$ e $B(T)$ dependem somente da temperatura $T$. Desde que a simetria relacionada com a mudança de sinal de $\phi$ é considerada, os termos de ordem ímpar podem ser excluídos.

Para formular uma transição contínua assumimos o valor de $\phi$ pequeno, de forma que possamos assim desconsiderar termos de ordem igual ou superior a 6. Consideramos também $f_{0}(T)=c$ (constante), $B(T)=d$ e $A(T)=a\left(T-T_{c}\right) / T_{c}$, com $a$ e $d$ sendo constantes positivas e $T_{c}$ representando a temperatura crítica. Com isso reescrevemos a expansão em (2.3.1) como

$$
f(T, \phi)=c+a\left(\frac{T}{T_{c}}-1\right) \phi^{2}+d \phi^{4} .
$$

Essa função descreve o comportamento de sistemas com transição de fase de contínua (vide Figura 2.3(a)). Fazendo $(\partial f / \partial \phi)_{T}$, obtemos os (dois) valores de mínimo para $T<T_{c}$.

Para formular uma transição de fase de primeira ordem fazemos $a<0$ e incluimos o termo $v \phi^{6}$. Considerando $v$ uma constante positiva, três mínimos em $T_{0}<T<T_{1}$ surgem na função (vide Figura 2.3(b)). O parâmetro de ordem salta descontinuamente em $T_{c}$, indo de $\phi= \pm \phi_{0}$ para zero. A temperatura crítica $T_{c}$ é alcançada quando os três mínimos têm a mesma profundidade.

Observamos que energias livre com dois ou mais poços como a desenhada na Figura 2.3 não são permitidas como energias livre de um sistema macroscópico. Uma expressão tal como (2.3.1) faz sentido apenas como um resultado grosseiro ("coarse-graining") [15]. A idéia de

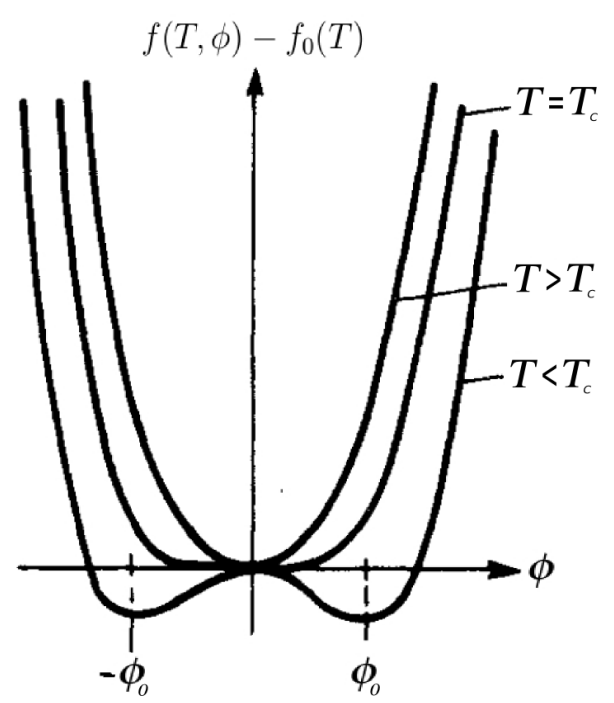

(a)

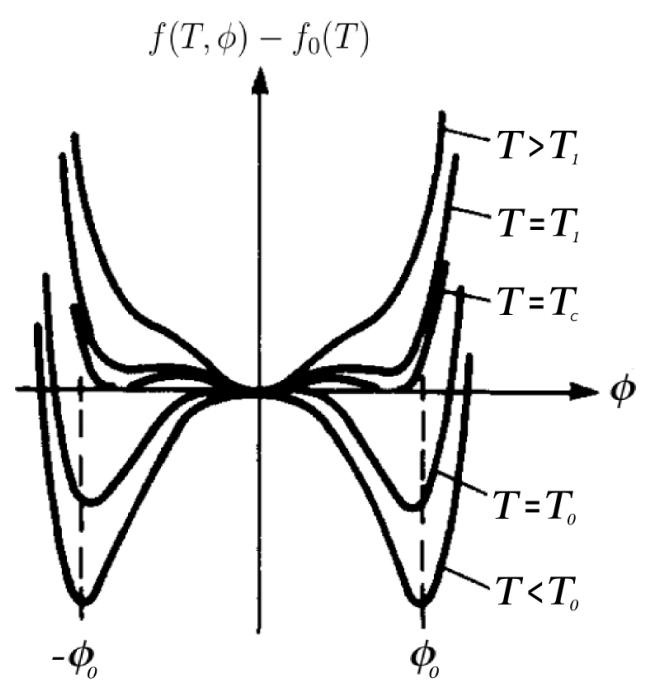

(b)

Figura 2.3: Gráficos da diferença da função de densidade de energia livre $f(T, \phi)-f_{0}(T)$ em função do parâmetro de ordem $\phi$ em diversas temperaturas. (a) Representação de transição de fase contínua. (b) Representação de transição de fase de primeira ordem. Figura adaptada de [15]. 
escrever uma densidade de energia livre grosseira é devida a Langer (1974). Em sua teoria, ele divide o sistema em células de tamanho $L^{d}$ estabelecendo o vínculo de que o parâmetro de ordem de cada célula esteja no intervalo $-\phi_{0}<\phi<\phi_{0}$. Agora, identificar $f(\phi)$ com a densidade de energia livre dos estados metaestável e instável não é totalmente satisfatório, pois $f(\phi)$ mostra alguma dependência no comprimento $L$. Quando temos $L \rightarrow \infty$ o comportamento esperado é o comportamento termodinâmico, ou seja, $f(\phi)$ torna-se uma função plana como a energia livre termodinâmica, porém, quando $L \leq \xi$, a função apresenta picos acentuados. Isso deve ocorrer, desde que as flutuações de comprimento de onda longo, as quais excedem o comprimento $L$, são suprimidas. Se fizermos $L \rightarrow \infty$ na região onde apenas uma fase aparece, então $f(\phi)$ tende à verdadeira densidade de energia $F(\phi)$. No entanto, se fizermos $L \rightarrow \infty$ na região de duas fases, a separação das fases é permitida em cada célula. Por causa de uma densidade de energia de superfície, as configurações que exibem mais de uma fase têm a densidade de energia livre diminuída em comparação com a densidade de energia livre das configurações que apresentam uma única fase.

Baseado nesse comportamento da energia livre, descrevemos abaixo um método numérico para a identificação da natureza das transições em sistemas de tamanho finito [19, 20]. Primeiramente consideramos $t$ como a distância reduzida ("campo de escala") que move o sistema de uma fase para outra. Se $t=0$ estamos no ponto crítico. Para $t<0$, a transição de fase é de primeira ordem e, para $t>0$, o sistema encontra-se na fase desordenada.

No limite termodinâmico, a energia livre $F_{L}(X, t) / L^{d}$ é independente de $X$ para $X_{1} \leq X \leq$ $X_{2}$ em uma transição de primeira ordem, mas para $L$ finito, a energia livre $F_{L}(X, T)$ tem uma estrutura de mínimos duplos com a expansão

$$
F_{L}(X, t)=L^{d} f_{0}(X, t)+L^{d-1} f_{1}(X, t)+\ldots,
$$

onde $X$ é um observável do sistema. Por exemplo, se a transição for guiada por um campo $H$, então o parâmetro de ordem $X$ é a magnetização $M$ do sistema; ou, se a transição for guiada pela temperatura $T$, então $X$ é a energia $E$ do sistema.

Para uma transição de fase de primeira ordem a densidade de energia livre $f_{0}$ é mínima e constante para $-X_{1} \leq X \leq X_{2}$. O termo $f_{1}$, o qual fornece a energia livre da superfície, tem um máximo em $X_{m}$. Com essa construção, de maneira análoga à teoria de Landau, a energia livre $F_{L}(X, T)$ terá dois mínimo em:

$$
\begin{aligned}
& X=X_{1}-O\left(L^{-1}\right) \\
& X=X_{2}-O\left(L^{-1}\right)
\end{aligned}
$$


e a altura do pico será

$$
\Delta F_{L}=F_{L}\left(X_{m}\right)-F_{L}\left(X_{1}\right) \simeq B(t) L^{d-1}
$$

Esse comportamento é sempre válido para $L \gg \xi$.

Quando $t \simeq 0$ e $L \ll \xi$, a energia livre $F_{L}(X, \beta)$ é dominada por sua parte singular e uma função universal das variáveis de escala $x$ e $y$ :

$$
\begin{aligned}
& x=X L^{\lambda_{x}}, \\
& y=t L^{\lambda_{t}} .
\end{aligned}
$$

Na transição (ponto crítico), definida por dois mínimos de igual profundidade, $\Delta F_{L}$ é uma função crescente de $-y$ no regime de primeira ordem desde que o mínimo se torne mais pronunciado.

Resumindo:

i. Se $\Delta F_{L}$ é independente de $L$ temos uma transição de fase contínua.

ii. Se $\Delta F_{L}$ crescer monotonamente com $L$ assim como uma estrutura que envolve a superposição de duas gaussianas para $L \gg \xi$, temos uma transição de fase contínua.

No regime $L \gg \xi$ o valor de $\Delta F_{L} \sim L^{d-1}$ torna-se difícil de ser calculado.

Como veremos na Capítulo 4, o número de vezes $N_{L}$ que um observável $X$, tal como a energia ou parâmetro de ordem, é amostrado em uma simulação de Monte Carlo onde são realizados $\mathcal{N}$ varreduras, é dado por

$$
N_{L}=N_{L}(X, T, \mathcal{N}) \simeq \mathcal{N} P_{L}(X, T)
$$

onde $P_{L}(X, T)$ é a probabilidade de amostrar esse observável definida pelo algoritmo. A quantidade $N_{L}(X, T, \mathcal{N})$ pode ser utilizada para construir um histograma em função de $X$. Como veremos, para o algoritmo de Metropolis, quanto maior for a quantidade de varreduras $\mathcal{N}$, mais a forma de $N_{L}(X, T, \mathcal{N})$ vai parecer com a probabilidade canônica de Gibbs.

A distribuição de probabilidade canônica de Gibbs nos diz que a probabilidade de obtermos um estado com o observável $X$ a uma dada temperatura $T$ é dada por [15]

$$
P_{L}(X, T) \propto \exp \left(-L^{d} f(X) / k_{B} T\right)
$$

Fazendo a analogia de $L^{d} f(X) / k_{B} T$ com uma "energia livre" $A_{L}(X, T, \mathcal{N})$, reescrevemos $N_{L}$ como

$$
N_{L}(X, T, \mathcal{N}) \equiv e^{-A_{L}(X, T, \mathcal{N})}
$$


que leva à seguinte relação:

$$
A_{L}(X, T, \mathcal{N})=-\ln \left[N_{L}(X, T, \mathcal{N})\right]
$$

Para uma temperatura fixa, a quantidade $A_{L}(X, T, \mathcal{N})$ difere da energia livre $F_{L}(X, T)$ apenas por uma quantidade aditiva que depende de $T, L$ e $\mathcal{N}$, mas não de $X$. Dessa forma, para $T, L$ e $\mathcal{N}$ fixos $(\mathcal{N} \gg 1)$, a forma de $A_{L}(X, T, \mathcal{N})$ será idêntica a forma de $F_{L}(X, T)$. Portanto, a medida de $\Delta A$ fornece um cálculo direto da correspondente $\Delta F$, ou seja,

$$
\Delta A_{L}=A_{L}(X)-A_{L}\left(X^{\prime}\right)=F_{L}(X)-F_{L}\left(X^{\prime}\right)=\Delta F_{L}
$$

Dessa maneira estabelecemos um método numérico para determinar a ordem da transição. 


\section{Capítulo 3}

\section{O modelo de Ising dipolar bidimensional}

Apresentamos nesta seção detalhes sobre o modelo de Ising dipolar bidimensional. Além de mostrar sua definição em termos de uma Hamiltoniana e evidenciar uma pequena diferença entre notações presentes na literatura, incluimos alguns resultados obtidos na literatura. Em particular, apresentamos dois diagramas de fase: um obtido através de simulações de Monte Carlo e outro obtido através da teoria de campo médio [21]. As características de cada uma das fases são apresentadas bem como uma discussão sobre a natureza das transições termodinâmicas que ocorrem entre elas.

\subsection{Descrição do modelo}

Assim como o modelo de Ising clássico, o modelo aqui considerado consiste em um modelo de spins interagentes localizados em uma rede regular bidimensional. Além disso, os spins podem orientar-se apenas em uma única direção (eixo $z$ ), o que confere à rede uma grande anisotropia uniaxial. Além da interação de troca usual entre os primeiros vizinhos, inclui-se nesse modelo interações entre os momentos de dipolo magnético dos spins. Dessa maneira, para um sistema com $N=L^{2}$ spins, escrevemos sua Hamiltoniana como:

$$
\mathcal{H}=\mathcal{H}_{\text {Ising }}+\mathcal{H}_{\text {dipolar }}
$$

onde

$$
\mathcal{H}_{\text {Ising }}=-\mathcal{J} \sum_{\langle i, j\rangle} S_{i} S_{j}
$$

e

$$
\mathcal{H}_{\text {dipolar }}=\frac{\mathcal{G}}{2} \sum_{i \neq j} \frac{\mu_{i} \mu_{j}}{R_{i j}^{3}} .
$$

Aqui $S_{i}$ representa o spin da $i$-ésima partícula, $\mathcal{J}$ é constante de interação de troca entre os primeiros vizinhos, $\mu_{i}$ representa o momento de dipolo magnético do $i$-ésimo spin, $\mathcal{G}$ é a 
constante de interação entre os momentos de dipolo magnético de dois spins $i$ e $j$ que estão à distância $R_{i j}=\left|\vec{R}_{i}-\vec{R}_{j}\right|$, com $\vec{R}_{i}$ sendo o vetor posição da $i$-ésima partícula. No primeiro somatório a notação $\langle i, j\rangle$ diz que apenas as interações entre os primeiros vizinhos devem ser consideradas. Entretanto, no segundo somatório considera-se as interações entre todos os pares de spins, mas o fator $1 / 2$ diz que a interação entre cada par $(i j)$ deve ser contabilizada apenas uma vez. Note que os acoplamentos $\mathcal{J}$ e $\mathcal{G}$ são independentes dos pares $(i j)$.

Resultados encontrados na literatura para as energias e temperaturas nesse modelo podem eventualmente apresentar discrepâncias. Isso é por causa de uma pequena diferença nas definições da Hamiltoniana. Apresentamos a seguir os aspectos que explicitam essa diferença e que ajudam a comparar e a esclarecer os resultados por nós obtidos. Consideramos primeiramente, para todo $i$ e $j$, as seguintes definições:

$$
\begin{aligned}
& S_{i}=\sigma_{i} S_{\mathrm{ef}}, \\
& \mu_{i}=\sigma_{i} \mu_{\mathrm{ef}},
\end{aligned}
$$

$\mathrm{e}$

$$
R_{i j}=a r_{i j}
$$

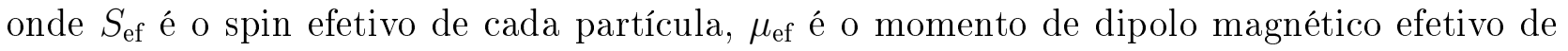
cada spin e $a$ é o parâmetro de rede, o qual define a distância entre dois pontos da rede onde estão localizadas as partículas. Os valores de $S_{\text {ef }}, \mu_{\mathrm{ef}}$ e $a$ dependem da composição específica de cada material. Como consideramos um sistema com spins de Ising, as variáveis $\sigma_{i}$ podem assumir somente os valores \pm 1 e $a$ é definido igual a 1 . Reescrevemos então a Hamiltoniana do sistema da seguinte maneira:

$$
\mathcal{H}=-\mathcal{J} S_{\mathrm{ef}}^{2} \sum_{\langle i, j\rangle} \sigma_{i} \sigma_{j}+\frac{\mathcal{G} \mu_{\mathrm{ef}}^{2}}{2 a^{3}} \sum_{i \neq j} \frac{\sigma_{i} \sigma_{j}}{r_{i j}^{3}},
$$

a qual pode ser multiplicada pela fator $2 a^{3} / \mathcal{G} \mu_{\mathrm{ef}}^{2}$ para fornecer:

$$
\frac{2 \mathcal{H} a^{3}}{\mathcal{G} \mu_{\mathrm{ef}}^{2}}=-\frac{2 \mathcal{J} a^{3}}{\mathcal{G}}\left(\frac{S_{\mathrm{ef}}}{\mu_{\mathrm{ef}}}\right)^{2} \sum_{\langle i, j\rangle} \sigma_{i} \sigma_{j}+\sum_{i \neq j} \frac{\sigma_{i} \sigma_{j}}{r_{i j}^{3}} .
$$

Considerando a seguinte mudança de variáveis,

$$
J=\mathcal{J} a^{3}\left(\frac{S_{\mathrm{ef}}}{\mu_{\mathrm{ef}}}\right)^{2} \quad \text { e } \quad g=\frac{\mathcal{G}}{2},
$$

e tendo em vista que a Hamiltoniana não depende explicitamente do tempo, escrevemos a 
energia de forma adimensional para uma dada configuração $\{\sigma\}$ do sistema da seguinte forma ${ }^{1}$ :

$$
\hat{E}(\{\sigma\})=-\frac{J}{g} \sum_{\langle i, j\rangle} \sigma_{i} \sigma_{j}+\sum_{i \neq j} \frac{\sigma_{i} \sigma_{j}}{r_{i j}^{3}} .
$$

Agora vamos definir

$$
\frac{J}{g}=2 \delta
$$

e escrever o somatório da interação dipolar como sendo duas vezes o somatório realizado somente sobre pares $(i j)$ distintos. Assim, obtemos uma relação entre as energias $\hat{E}=2 E$, com a nova energia $E$ sendo escrita como

$$
E(\{\sigma\})=-\delta \sum_{\langle i, j\rangle} \sigma_{i} \sigma_{j}+\sum_{i<j} \frac{\sigma_{i} \sigma_{j}}{r_{i j}^{3}} .
$$

Desse modo caracterizamos nosso sistema em termos de um único parâmetro $\delta$ dado pela razão dos acoplamentos das interações de troca e dipolar.

Considerando que a probabilidade canônica de encontrarmos a configuração $\{\sigma\}$ seja a mesma para qualquer uma das duas definições, devemos preservar a igualdade $\hat{\beta} \hat{E}=\beta E$. Utilizando que $\hat{E}=2 E, \hat{\beta}=1 / k_{B} \hat{T}$ e $\beta=1 / k_{B} T$, obtemos finalmente a seguinte relação:

$$
\hat{T}=2 T \text {. }
$$

Observamos que $\hat{T}$ e $T$ têm dimensão $\left[k_{B}\right]^{-1}$, assim elas não são estritamente as temperaturas absolutas observadas nos sistemas termodinâmicos. Mas, por convenção, chamamos $T$ (ou $\hat{T}$ ) de temperatura em simulações computacionais, nas quais são utilizadas unidades naturais, onde a constante de Boltzmann $k_{B}$ é assumida igual a 1. Na literatura, os trabalhos geralmente expõem seus resultados utilizando essa convenção, por isso algumas diferenças podem ser observadas. Como vimos, as diferenças ocorrem dependendo da definição da soma do termo de interação dipolar: se a soma é sobre todos os pares ou apenas sobre os pares distintos. De qualquer forma, a relação (3.1.7) entre os acoplamentos e a relação (3.1.9) entre as temperaturas devem ser sempre observadas. Nesta dissertação adotaremos a energia adimensional $E$ definida pela expressão (3.1.8), descrita pela razão $\delta$ entre os acoplamentos, e a temperatura $T$.

Definimos $^{2}$, como proposto primeiramente em [12], o grau de orientação de uma dada configuração $\{\sigma\}$ do sistema como:

$$
O_{h v}=\left|\frac{n_{h}-n_{v}}{n_{h}+n_{v}}\right|,
$$

\footnotetext{
${ }^{1}$ A notação $\{\sigma\}$ corresponde ao conjunto de todas as $N$ variáveis $\sigma$ do sistema, ou seja, corresponde ao conjunto $\left\{\sigma_{1}, \sigma_{2}, \ldots, \sigma_{N}\right\}$.

${ }^{2}$ Embora haja outra possibilidade como definido em [22, 23].
} 
onde as quantidades $n_{h}$ e $n_{v}$ denotam, respectivamente, o número de ligações ${ }^{3}$ horizontais e verticais entre spins alinhados antiparalelamente. Como veremos, esse parâmetro de ordem permite uma melhor caracterização das fases observadas à medida que a temperatura varia.

\subsection{Diagrama de fase}

A presença da interação dipolar altera completamente o comportamento do sistema em relação ao comportamento do modelo de Ising bidimensional clássico. A situação na qual temos a razão $\delta$ positiva corresponde a interações de troca ferromagnéticas e interações dipolares antiferromagnéticas. Interações ferromagnéticas favorecem o alinhamento dos spins em uma mesma direção, pois este alinhamento diminui a energia do sistema. Por outro lado, interações antiferromagnéticas diminuem a energia do sistema quando os spins estão alinhados antiparalelamente. Percebemos, dessa maneira, a existência de competição entre os dois tipos de interação. A presença dessa competição atribui ao modelo características não usuais, as quais, como veremos, tornam seu diagrama de fase bastante rico.

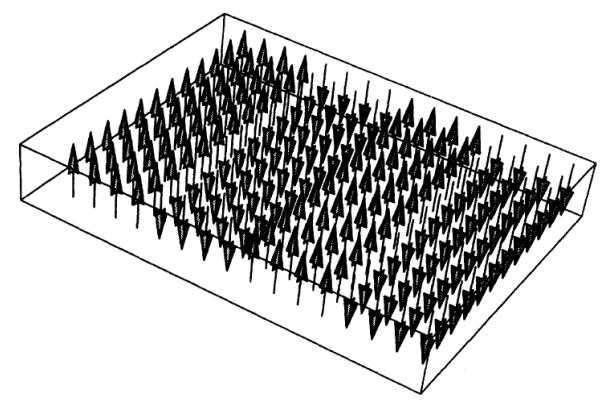

Figura 3.1: Representação da estrutura de domínio do tipo faixas de spins com largura $h=4$ para o estado fundamental. Figura retirada de [24].

De um ponto de vista teórico, uma interação repulsiva $1 / r^{s}$ para sistemas em $d$ dimensões, com $d<s \leq d+1$, adicionada à interação entre primeiros vizinhos ferromagnética usual, leva a ausência de ferromagnetismo para todas as temperaturas [25, 26, 27]. De fato, o estado fundamental ferromagnético do modelo de Ising clássico é alterado para uma série de fases do tipo faixas, as quais consistem em domínios ferromagnéticos de largura $h$ com magnetizações opostas, como mostrado na Figura 3.1. Cálculos analíticos da energia do estado fundamental [28, 24], argumentos baseados no grupo de renormalização [29], abordagens via teoria de campo médio [21], aproximações de modelos contínuos [30] e simulações de Monte Carlo [31, 12], predizem a existência da fase tipo faixas de spins a baixas temperaturas, sendo que a largura

\footnotetext{
${ }^{3}$ Somente as ligações entre primeiros vizinhos devem ser consideradas.
} 


\begin{tabular}{cc}
\hline \hline$h$ & $\delta$ \\
\hline 1 & $0,440313-1,258539$ \\
2 & $1,258539-2,172456$ \\
3 & $2,172456-2,814495$ \\
4 & $2,814495-3,304242$ \\
5 & $3,304242-3,699024$ \\
6 & $3,699024-4,029349$ \\
7 & $4,029349-4,313172$ \\
8 & $4,313172-4,561907$ \\
9 & $4,561907-4,783242$ \\
10 & $4,783242-4,982600$ \\
\hline \hline
\end{tabular}

Tabela 3.1: Resultados de cálculos analíticos para as regiões de equilíbrio em $\delta$ para a largura $h$ das faixas. Dados retirados de [32].

das faixas depende da razão $\delta$. Cálculos analíticos mostram que essas fases do tipo faixas ocorrem em uma região do diagrama de fase onde a razão $\delta$ assume valores maiores que $\delta^{*}$, com $\delta^{*}=0,425$, conforme Ref. [24] e $\delta^{*}=0,440313$, conforme Ref. [32]. O segundo valor também pode ser encontrado em [21], no qual define-se a seguinte expressão assintótica para a largura das faixas: $h=h(\delta) \approx e^{\delta / 2}$. Portanto, para o estado fundamental $(T=0)$, conforme aumentamos o valor de $\delta$ a largura $h$ das faixas aumenta, característica esta também presente nos resultados obtidos através de cálculos analíticos apresentados em [24]. Cálculos analíticos mais recentes sobre essa característica foram apresentados em [32] e são mostrados na Tabela 3.1 .

A espessura $h$ é uma escala natural desses sistemas. Assim, as simulações devem ser feitas para sistemas com tamanhos $L>h$. Experimentalmente observa-se que a largura das faixas para um filme de Fe sobre $\mathrm{Cu}(100)$ é da ordem de $1 \mu m$ [6], o que corresponde a $h=4000$ constantes de rede [21]. Utilizando a relação $h(\delta)=e^{\delta / 2}$, obtemos $\delta=2 \ln (4000) \approx 16,6$. Notamos que os trabalhos presentes na literatura utilizam valores de $\delta$ aproximadamente uma ordem de grandeza menores do que o estimado para sistemas experimentais.

Reproduzimos na Figura 3.2 o diagrama de fase no plano $(T-\delta)$ apresentado em [21]. Este diagrama $^{4}$ foi obtido por meio de simulações de Monte Carlo para redes de tamanho $L \approx 50$. Os resultados obtidos a partir das simulações de Monte Carlo mostram um comportamento semelhante àquele previsto por cálculos analíticos, ou seja, conforme $\delta$ aumenta, a largura da faixa $h$ também aumenta. Note que os domínios do tipo faixas permanecem estáveis mesmo a temperaturas não nulas. De uma maneira grosseira, pode-se dizer que, a partir

\footnotetext{
${ }^{4}$ Outro diagrama de fase menos detalhado é apresentado em [32] para uma rede de tamanho $L=48$ e pode servir para uma eventual comparação.
} 
de certas temperaturas de transição, cessa a presença dos domínios tipo faixas e uma fase menos ordenada aparece. Notamos pela Figura 3.2 a existência de quatro tipos de transições à medida que aumentamos a temperatura:

i) uma transição contínua em $T_{c}^{(2)}(\delta)$ (círculos pretos) para $\delta \lesssim 0,9$. Observa-se que para $\delta \lesssim \delta^{*}$ essa transição ocorre a partir de uma fase semelhante a uma fase antiferromagnética (e não de uma fase do tipo faixas) para uma fase desordenada;

ii) uma transição direta (sem fase intermediária) em $T_{c}^{(2)}(\delta)$ (triângulos vermelhos) para $\delta \gtrsim 0,9$ entre fases do tipo faixas e uma fase do tipo líquido tetragonal;

iii) uma transição entre fases do tipo faixas e fases nemáticas em $T_{c}^{(1)}(\delta)$ ("diamantes" verdes) para regiões estreitas de $\delta$;

iv) uma transição de fase entre as fases nemática e tetragonal em $T_{c}^{(2)}(\delta)$ (triângulos vermelhos) também para regiões estreitas de $\delta$.

A altas temperaturas, o sistema deixa a fase tetragonal e vai para uma fase paramagnética totalmente desordenada. No entanto, nenhuma transição bem definida entre as fases tetragonal

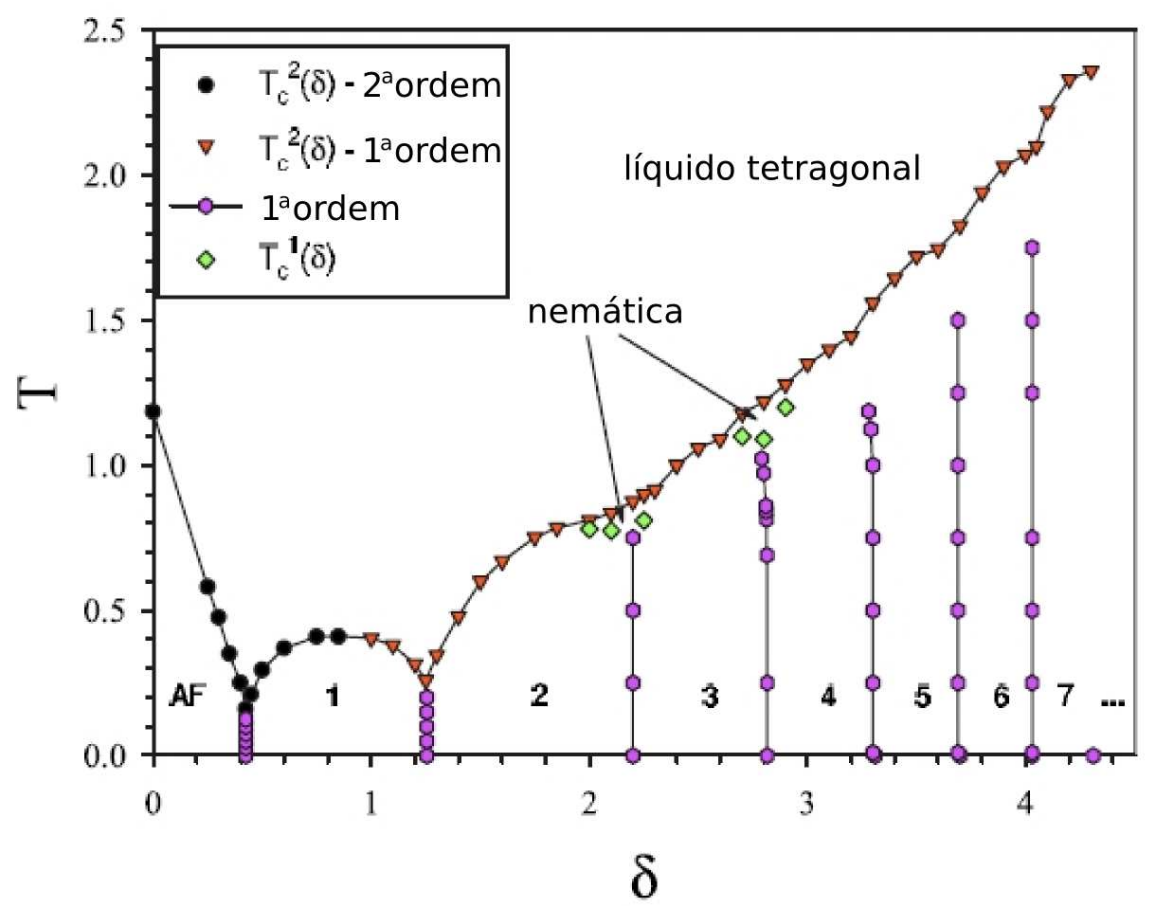

Figura 3.2: Diagrama de fase obtido através de simulações numéricas de Monte Carlo. Os números de 1 a 7 representam a largura das faixas $h$ para cada uma das fases correspondentes separadas pelos círculos roxos. Figura adaptada de [21]. 
e paramagnética é observada em simulações de Monte Carlo. Isso contrasta com o resultado do trabalho de Abanov e coautores [30] que, baseado num modelo contínuo, prediz uma linha de transição bem definida entre essas duas fases. Nesse mesmo trabalho, Abanov e coautores indicam a existência de dois tipos de cenários, descritos como:

i) uma transição de fase direta da fase do tipo faixas para a fase tetragonal;

ii) duas transições de fase, com uma fase nemática intermediária.

A presença desses dois tipos de cenários também foi comprovada através de resultados obtidos por meio de simulações de Monte Carlo [33, 32]. Cannas e coautores [33] foram os primeiros a realizar um estudo que identificasse, por meio de simulações numéricas, a fase nemática. Sobre a transição de fase faixas-nemática em $T_{c}^{(1)}$ o trabalho mostra, através de um estudo de escala de tamanho finito, que os resultados concordam com o esperado para uma transição de fase de primeira ordem. Entretanto, ressalvas são feitas devido a dois fatos os quais são incomuns para uma transição de primeira ordem no limite termodinâmico: a energia torna-se contínua e uma saturação no máximo do calor específico é observada. Isso sugere um mecanismo do tipo Kosterlitz-Thoulosse [11, 34], o que concordaria com a predição feita por Abanov e coautores [30].

Com respeito à transição de fase nemática-tetragonal em $T_{c}^{(2)}$, Cannas e coautores [33] argumentam que a mudança descontínua do paràmetro de ordem orientacional sugere uma transição de fase de primeira ordem. Seus resultados são inconclusivos e os justificam por causa da presença de fortes efeitos de tamanho finito. Argumentam ainda que o comportamento obtido é mais complexo que o esperado por Abanov e coautores [30], que previram uma transição de fase contínua. Desde que toda a análise de Abanov e coautores [30] é baseada em argumentos de campo médio, o desacordo com os resultados de Monte Carlo pode ser entendido a partir da natureza de indução por flutuações da transição. Por outro lado, a aproximação de Hartree da versão de Landau-Gizburg do modelo prediz uma transição de fase de primeira ordem induzida por flutuações para qualquer valor de $\delta$ [35].

De fato, as simulações de Monte Carlo mostram um cenário complexo, onde a natureza da transição de fase ordem-desordem em $T_{c}^{(2)}$ depende do valor de $\delta$. Rastelli e coautores [36] mostraram que para $\delta=0$, a transição é contínua e pertence à classe de universalidade do modelo de Ising de primeiros vizinhos. Eles também encontraram evidências para uma transição contínua em $\delta=0,85(h=1)$, mas com valor para o expoente crítico $\beta=0,08$ não usual. No entanto, Cannas e coautores [33] mostraram que, para $\delta=1$, o sistema apresenta uma transição de primeira ordem fraca. Esses resultados são consistentes com a presença de 
uma linha de transição contínua para pequenos valores de $\delta$ que liga-se através de uma linha contínua a uma linha de transição de primeira ordem para valores maiores de $\delta$ em algum ponto tricrítico localizado entre 0,85 e 1,00, como mostrado no diagrama da Figura 3.2. O expoente crítico não usual em $\delta=0,85$ pode ser devido ao efeito de cruzamento próximo ao ponto tricrítico. Existem evidências de que a transição em $T_{c}^{(2)}(\delta)$ é de primeira ordem para $\delta=2[33,35]$ e para $\delta=1,7(h=2)$ e 2,5 $(h=3)$ (primeira ordem fraca) [36]. Booth e coautores [12], assim como Rastelli e coautores [36], com resultados obtidos do calor específico e parâmetro de ordem para $\delta=3$ e $\delta=4,45(L=64)$, sugerem que a transição em $T_{c}^{(2)}(\delta)$ torna-se contínua novamente. Entretanto, esse é um assunto ainda não muito bem esclarecido. Resultados numéricos baseados em análises de séries temporais, para o mesmo parâmetro $(\delta=4,45)$ e tamanho de rede $(L=64)$, indicam que a transição é de primeira ordem [23].

Rastelli e coautores [32] realizaram um estudo utilizando cálculos analíticos para o estado fundamental, o qual mostra que uma outra classe de configurações do tipo tabuleiro $\langle 1, n\rangle$ aparece em uma região estreita do diagrama de fase onde $0<\delta<0.4403$. A Figura 3.3 mostra como são as configurações típicas dessas fases. Nesse mesmo trabalho, Rastelli e coautores apresentam resultados de simulações de Monte Carlo para temperatura finita que também confirmam a existência dessas fases do tipo tabuleiro. A Tabela 3.2 mostra as regiões de estabilidade para cada uma das configurações.

Ressaltamos aqui dois aspectos que foram poucos explorados na literatura até o momento:

i. O caso limite onde $\delta$ é muito grande, ou seja, quando a intensidade da interação de troca é muito maior que a intensidade da interação dipolar e espera-se recuperar o limite do modelo de Ising clássico. MacIsaac e coautores [24] realizaram cálculos analíticos e Rastelli e coautores [32] efetuaram simulações numéricas de Monte Carlo.

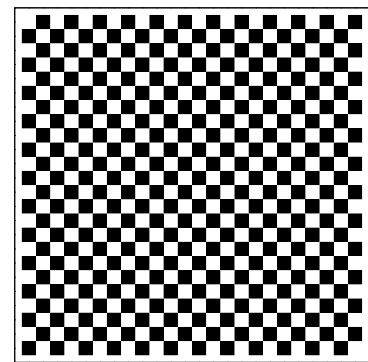

(a) $\langle 1,1\rangle$

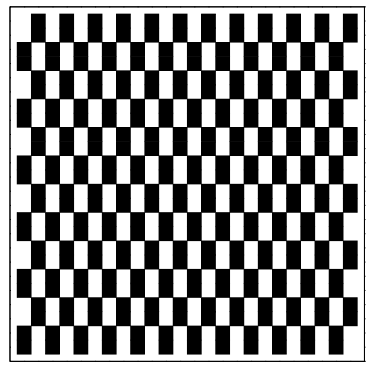

(b) $\langle 1,2\rangle$

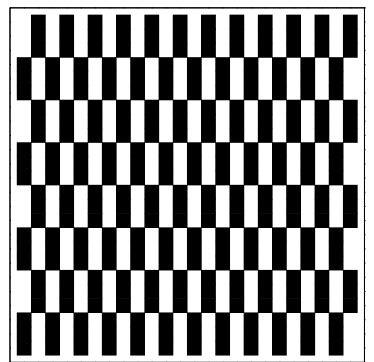

(c) $\langle 1,3\rangle$

Figura 3.3: Configurações de spins para a região $\delta<0,4403$. Fases do tipo tabuleiro. Figura adaptada de [32]. 
ii. O estudo onde o sistema é submetido a um campo magnético externo. Arllet e coautores [37] realizaram este estudo apenas para $\delta=3$ e 4, 45, e verificaram que, para esses casos, o diagrama de fase no plano $(H-T)$ condiz qualitativamente com o previsto por Abanov e coautores [30].

A abordagem de campo médio descarta flutuações e pode introduzir mudanças qualitativas no comportamento crítico. Contudo, é importante comparar as simulações de Monte Carlo com a abordagem de campo médio no sentido de estabelecer os limites de validade da aproximação. A abordagem de campo médio leva a um número infinito de equações acopladas que, exceto em situações particulares, não podem ser resolvidas exatamente. Para temperaturas subcríticas, qualquer propriedade tem que contar com soluções numéricas das equações de campo médio ou, fora isso, um ansatz deve ser introduzido, para, ainda assim, obter soluções aproximadas das equações de campo médio [21].

Na Figura 3.4 mostramos o digrama de fase obtido via abordagem de campo médio num estudo feito por Pighín e Cannas [21]. Embora a aparência geral seja a mesma do diagrama apresentado na Figura 3.2, algumas diferenças devem ser notadas. A primeira diferença é a falta dos ordenamentos nemático e tetragonal para a teoria de campo médio. O problema é que essas duas fases também são desordenadas. Desde que flutuações são desprezadas na aproximação de campo médio, a única solução desordenada possível é a fase paramagnética. Por outro lado, a aproximação de campo médio apresenta soluções de estados híbridos nas regiões do diagrama de fase onde as simulações de Monte Carlo predizem somente o ordenamento nemático. Como discutido por Pighín e Cannas [21], os estados híbridos são instáveis naquela região, sugerindo que (na linguagem do grupo de renormalização) as flutuações têm grande importância. De alguma forma, os estados híbridos poderiam ser os estados mais próximos do estado nemático possíveis de se obter quando as flutuações são desprezadas. Isso pode ser consistente com o fato das flutuações, quando incluídas, poderem modificar a natureza contínua da predição da teoria de campo médio para a transição ordem-desordem.

Por fim, observamos que, ao contrário da predição de campo médio, as linhas de transição

\begin{tabular}{cc}
\hline \hline$h$ & $\delta$ \\
\hline$\langle 1,1\rangle$ & $0.000000-0.415203$ \\
$\langle 1,2\rangle$ & $0.415203-0.439420$ \\
$\langle 1,3\rangle$ & $0.439420-0.440279$ \\
$\langle 1,4\rangle$ & $0.440279-0.440311$ \\
\hline \hline
\end{tabular}

Tabela 3.2: Regiões de estabilidade para as fases do tipo $\langle 1 . n\rangle$. Dados retirados de [32]. 


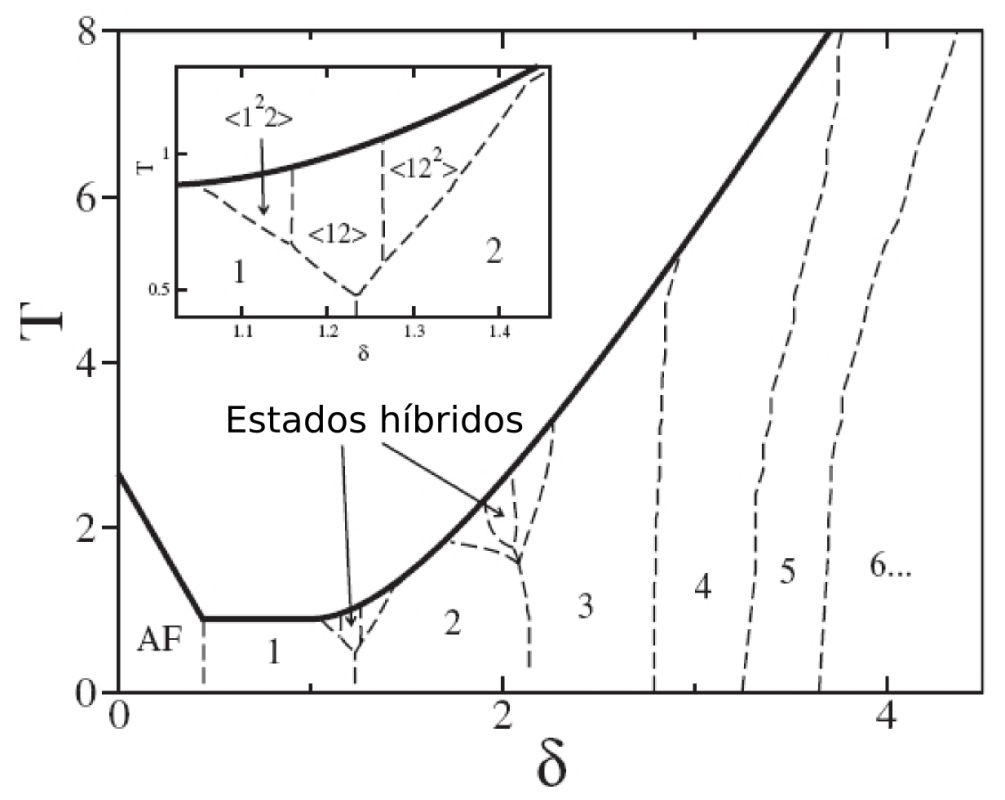

Figura 3.4: Diagrama de fase obtido via teoria de campo médio. Os números de 1 a 6 representam as regiões onde a solução mostra a presença de fases do tipo faixas com larguras correspondentes ao valor indicado. Figura retirada de [21].

entre as fases de faixas são completamente verticais no diagrama feito a partir das simulações de Monte Carlo. Isto implica na não dependência da largura das faixas em relação à temperatura, ao menos até valores de $\delta=4$. Para valores de $\delta$ maiores, Pighín e Cannas [21] sugerem que as flutuações possam provocar no sistema uma transição para um "regime de campo médio", onde a largura das faixas começa a exibir uma dependência com a temperatura. Notamos que um estudo que comprove isso também ainda não foi realizado. 


\section{Capítulo 4}

\section{Métodos de Monte Carlo}

Nesta seção vamos discutir conceitos teóricos envolvidos nos algoritmos de simulação de Monte Carlo utilizados nessa dissertação. Uma breve revisão sobre conceitos em Mecânica Estatística de equilíbrio, em particular, sobre conceitos da estatística canônica, é apresentada. Descrevemos aspectos básicos da teoria das cadeias de Markov, incluindo uma apresentação sobre o algoritmo de Metropolis e o tempo de autocorrelação integrado. Por fim, apresentamos a formulação do algoritmo multicanônico, incluindo detalhes sobre as relações recursivas.

\subsection{Conceitos em Mecânica Estatística}

Consideramos aqui apenas sistemas em equilíbrio térmico descritos por meio do ensemble canônico. Para este ensemble existem relações muito simples entre o comportamento macroscópico dos sistemas e as suas propriedades microscópicas.

Consideremos um sistema magnético fechado com um número de partículas $N$ constante $^{1}$, submetido a um campo externo $H$, e em equilíbrio com um banho térmico a uma temperatura T. Para definirmos a distribuição de probabilidades de Gibbs, a qual define a probabilidade do sistema estar em um microestado específico, denotado por $k$, utilizamos o peso de Boltzmann, que é dado por

$$
w_{B}(k, \beta) \equiv e^{-\beta \mathcal{H}_{k}}
$$

onde $\mathcal{H}_{k}=\mathcal{H}\left(\{\sigma\}_{k}\right)$ é a(o) Hamiltoniana(o) do sistema para uma dada configuração ${ }^{2}\{\sigma\}_{k}=$ $\left\{\sigma_{1}, \sigma_{2}, \ldots, \sigma_{f N}\right\}_{k}$ e $\beta=1 / k_{B} T$.

Considerando a probabilidade canônica $p_{B}(k, \beta)$ de encontrar um microestado com confi-

\footnotetext{
${ }^{1}$ Como sistemas magnéticos são geralmente descritos por sólidos, nós assumiremos que o volume do sistema $V$ é constante e proporcional ao número de partículas $N$, dessa forma omitiremos também esses parâmetros das relações termodinâmicas subsequentes.

${ }^{2}$ Aqui $f$ representa o número de graus de liberdade por partícula.
} 
guração $\{\sigma\}_{k}$ proporcional ao peso de Boltzmann e impondo a condição de normalização

$$
\sum_{k=1}^{\mathcal{N}} p_{B}(k, \beta)=1
$$

obtemos

$$
p_{B}(k, \beta)=\frac{w_{B}(k, \beta)}{\mathcal{Z}(\beta)} .
$$

$\mathcal{Z}(\beta)$ é a função de partição canônica, dada explicitamente por

$$
\mathcal{Z}(\beta)=\sum_{k=1}^{\mathcal{N}} e^{-\beta \mathcal{H}_{k}}
$$

com a soma sobre $k$ sendo feita sobre todos os $\mathcal{N}$ microestados acessíveis ao sistema.

Considerando a probabilidade canônica dada pela expressão (4.1.3), a média térmica de uma grandeza observável $\langle\mathcal{O}\rangle(\beta)$ é escrita como

$$
\langle\mathcal{O}\rangle(\beta)=\sum_{k=1}^{\mathcal{N}} \mathcal{O}_{k} p_{B}(k, \beta),
$$

onde $\mathcal{O}_{k}$ é uma grandeza observável (e.g. magnetização, energia interna, parâmetro de ordem, etc) para uma dada configuração $\{\sigma\}_{k}$.

Em geral, modelos clássicos apresentam Hamiltonianas descritas por variáveis contínuas, o que implica em um número infinito de microestados acessíveis e em substituir os somatórios sobre $k$ nas expressões (4.1.2), (4.1.4) e (4.1.5) por integrais múltiplas realizadas sobre todos as variáveis $\sigma_{i}$, para $i=1, \ldots, N$. No entanto, para um modelo contituído de spins de Ising, isto é, onde as variáveis $\sigma_{i}(i=1, \ldots, N)$ podem assumir somente dois valores, \pm 1 , temos exatamente $\mathcal{N}=2^{N}$ microestados acessíveis. No modelo de Ising puro os principais observáveis são a energia e a magnetização. Nesse caso, tanto os valores que podem ser assumidos pela energia quanto pela magnetização são valores discretos e igualmente espaçados. Contudo, mesmo para um sistema constituído de spins de Ising, os observáveis $\mathcal{O}_{k}$ podem assumir valores que não são igualmente espaçados, além disso, esses valores dependem fortemente do número de partículas $N$, como é o caso de sistemas com interação de longo alcance (e.g., o modelo de Ising dipolar).

Em simulações numéricas, quando temos valores contínuos (ou quase contínuos) para os observáveis, como é o caso de modelos descritos por Hamiltonianas clássicas, a questão é mais sútil. Nesse caso, é conveniente discretizar o espaço de valores que podem ser assumidos pelos observáveis, em especial pela energia ${ }^{3}$. Para tanto, dividimos o intervalo dos valores admitidos

\footnotetext{
${ }^{3}$ Como veremos, algoritmos que utilizam histogramas como o algoritmo multicanônico exigem essa discretização.
} 
pela energia em $M+1$ fatias de tamanho $\epsilon$. Isso é feito para que uma energia $E_{\eta}$ represente todas as energias encontradas no $\eta$-ésimo intervalo ${ }^{4}$. Assim, todas as energias $E$ que estão no intervalo $E_{\eta} \leq E<E_{\eta+1}$ serão consideradas para o valor da degenerescência $\Omega\left(E_{\eta}\right)$. Definimos então, para essa discretização, os valores de $E_{\eta}$ da seguinte maneira:

$$
E_{\eta}=E_{0}+\eta \epsilon
$$

com $\eta=0,1,2, \ldots M$ e $E_{0}$ é a energia mínima que o sistema pode assumir. Na prática determinamos $\epsilon$ como o menor intervalo de energia de modo que o valor de $\Omega\left(E_{\eta}\right)$ não seja nulo, seja qual for o valor de $\eta$ e, em geral, escolhemos $M$ da ordem do número de partículas $N$ do sistema.

Note que, em geral, existe uma degenerescência nos valores das quantidades observáveis, ou seja, existe um número $\Omega\left(E_{l}, \mathcal{O}_{m}\right)$ de configurações distintas associadas às diferentes energias as quais fornecem um mesmo observável $\mathcal{O}_{m}$. Considerando isso, reescrevemos o peso de Boltzmann como

$$
w_{B}\left(\mathcal{O}_{m}, \beta\right)=\sum_{l=1}^{n_{m}} \Omega\left(E_{l}, \mathcal{O}_{m}\right) e^{-\beta E_{l}} .
$$

Este peso é utilizado para definir a probabilidade de encontrar um observável $\mathcal{O}_{m}$ :

$$
p_{B}\left(\mathcal{O}_{m}, \beta\right)=\frac{w_{B}\left(\mathcal{O}_{m}, \beta\right)}{\mathcal{Z}(\beta)}
$$

onde $n_{m}$ é o número de energias associadas a um observável $\mathcal{O}_{m}$. A função de partição então é dada por

$$
\mathcal{Z}(\beta)=\sum_{m=0}^{\mathcal{M}_{\mathcal{O}}} \sum_{l=1}^{n_{m}} \Omega\left(E_{l}, \mathcal{O}_{m}\right) e^{-\beta E_{l}},
$$

onde a primeira soma é sobre todos os $\mathcal{M}_{\mathcal{O}}+1$ valores admitidos para o observável $\mathcal{O}$. Assim, a média térmica para o observável é dada por

$$
\langle\mathcal{O}\rangle(\beta)=\sum_{m=0}^{\mathcal{M}_{\mathcal{O}}} \mathcal{O}_{m} p_{B}\left(\mathcal{O}_{m}, \beta\right) .
$$

Vale notar que essa expressão fornece exatamente o mesmo resultado para a média do observável $\mathcal{O}$ que a expressão (4.1.5).

Quando o observável for a energia da configuração, teremos $\Omega\left(E_{l}, E_{m}\right)=\delta_{l, m} \Omega\left(E_{m}\right)$, assim o somatório sobre $l$ na equação (4.1.7) desaparece e o peso de Boltzmann fica escrito como

$$
w_{B}\left(E_{m}, \beta\right)=\Omega\left(E_{m}\right) e^{-\beta E_{m}} .
$$

\footnotetext{
${ }^{4}$ Aqui $\eta$ representa um índice inteiro.
} 
Este peso é utilizado para definir a probabilidade canônica de encontrar o sistema em uma energia $E_{m}$ como

$$
p_{B}\left(E_{m}, \beta\right)=\frac{w_{B}\left(E_{m}, \beta\right)}{\mathcal{Z}(\beta)},
$$

sendo a função de partição canônica dada por

$$
\mathcal{Z}(\beta)=\sum_{m=0}^{\mathcal{M}} w_{B}\left(E_{m}, \beta\right) .
$$

A soma sobre $m$ indica que todos os $\mathcal{M}+1$ valores possíveis para a energia $E_{m}$ devem ser $\operatorname{considerados}^{5}$ e a degenerescência $\Omega\left(E_{m}\right)$ determina o número de configurações associadas à energia $E_{m}$.

A média térmica para o observável $E_{m}$ fornece a energia média para um determinado valor de $\beta$ e é dada pela expressão

$$
\langle E\rangle(\beta)=\sum_{m=0}^{\mathcal{M}} E_{m} p_{B}\left(E_{m}, \beta\right) .
$$

Como a energia não se encontra sempre no seu valor de equilíbrio, consideramos também as suas flutuações

$$
\sigma^{2}(E)=\left\langle(E-\langle E\rangle)^{2}\right\rangle=\left\langle E^{2}\right\rangle-\langle E\rangle^{2}
$$

Um resultado bastante conhecido [38], é a expressão para o calor específico em função das flutuações na energia:

$$
C_{v}(\beta)=\frac{k_{B} \beta^{2}}{N}\left[\left\langle E^{2}\right\rangle-\langle E\rangle^{2}\right]
$$

$\operatorname{com}\langle E\rangle=\langle E\rangle(\beta)$.

Em particular, para o caso específico do modelo de Ising dipolar bidimensional, definimos aqui também a susceptibilidade do parâmetro de ordem $O_{h v}$ como

$$
\chi\left(O_{h v}\right) \equiv N\left[\left\langle O_{h v}^{2}\right\rangle-\left\langle O_{h v}\right\rangle^{2}\right]
$$

Vale notar que as médias térmicas das grandezas microscópicas, as quais fornecem os valores das grandezas termodinâmicas do sistema físico em equilíbrio térmico, podem ser obtidas através da função de partição. Por exemplo, as médias térmicas das expressões (4.1.14) e (4.1.16) podem ser obtidas derivando a função de partição, ou seja,

$$
\langle E\rangle(\beta)=-\left(\frac{\partial}{\partial \beta} \ln \mathcal{Z}(\beta)\right)_{H, N}
$$

\footnotetext{
${ }^{5}$ Quanto melhor for a discriminação entre as energias mais $M$ tenderá à $\mathcal{M}$.
} 
e

$$
C_{v}(\beta)=\frac{k_{B} \beta^{2}}{N}\left(\frac{\partial^{2}}{\partial \beta^{2}} \ln \mathcal{Z}(\beta)\right)_{H, N} .
$$

Entretanto, são raros os casos em que temos resultados analíticos exatos para a função de partição. Como alternativa, utilizamos os métodos de Monte Carlo para amostrar configurações do sistema de maneira significativa e com isso, obter estimadores adequados para as médias térmicas das grandezas físicas observáveis.

\subsection{Cadeias de Markov}

O método de Monte Carlo para amostrar a distribuição de probabilidades de Gibbs envolve um elemento de chance. Números aleatórios (ou, mais estritamente, números pseudo aleatórios) são utilizados para selecionar as configurações do sistema. Para entender como isso funciona, precisamos inicialmente abordar um pouco da teoria geral dos "processos Markovianos" $[39,16]$.

Um processo de Markov é uma regra para gerar randomicamente uma nova configuração para um sistema a partir da configuração presente. Um fato importante sobre essa regra é que ela deve depender somente do presente estado do sistema, e não deve requerer qualquer conhecimento prévio. Nós podemos expressar essa regra na forma de um conjunto de probabilidades; para cada par possível de estados $\eta$ e $\eta^{\prime}$, existe uma probabilidade associada, $P\left(\eta \rightarrow \eta^{\prime}\right)$, de que o sistema estando inicialmente no estado $\eta$, estará no estado $\eta^{\prime}$ no próximo passo. Essas probabilidades satisfazem uma regra de soma a qual expressa o fato de que a cada passo o sistema precisa ir para algum estado:

$$
\sum_{\eta^{\prime}} P\left(\eta \rightarrow \eta^{\prime}\right)=1
$$

Nós estamos interessados em produzir uma cadeia de Markov — uma sequência de estados gerados por um processo de Markov - na qual a frequência de ocorrência de um estado $\eta$ é proporcional a probabilidade de Gibbs $p_{\eta}$ associada. Para isso, precisamos estabelecer duas condições sobre as probabilidades de transição $P\left(\eta \rightarrow \eta^{\prime}\right)$.

(i) A partir de uma configuração, deve ser possível ir para qualquer outra configuração aplicando uma regra de evolução por tantas vezes quanto necessárias. Isso é chamado frequentemente de hipótese da acessibilidade.

(ii) As probabilidades de transição devem satisfazer a microrreversibilidade ou condição de balanço detalhado:

$$
p_{\eta} P\left(\eta \rightarrow \eta^{\prime}\right)=p_{\eta^{\prime}} P\left(\eta^{\prime} \rightarrow \eta\right)
$$


Nós podemos encontrar conjuntos de probabilidades de transição as quais obedecem essas condições para qualquer distribuição de Gibbs $p_{\eta}$ : podemos escolher $P\left(\eta \rightarrow \eta^{\prime}\right) \propto e^{\beta\left(E_{\eta}-E_{\eta^{\prime}}\right)}$, por exemplo. No entanto, a maioria desses conjuntos de probabilidades de transição não satisfaz essas condições e, como consequência, elas não são utéis para modelar estados descritos pela distribuição de Gibbs.

Supondo que nós escolhemos $P$ tal que (i) e (ii) acima são satisfeitas, podemos mostrar dois resultados. Primeiro, nós provamos que se cada configuração $\eta$ aparece em um passo $n$ da cadeia de Markov com probabilidade $W(\eta, n)$ igual a sua probabilidade de Gibbs,

$$
W(\eta, n)=p_{\eta}
$$

então ela também aparece com essa probabilidade no passo $n+1$. Isso segue porque a distribuição no passo $n+1$ será

$$
\begin{aligned}
W(\eta, n+1) & =\sum_{\eta^{\prime}} p_{\eta} P\left(\eta^{\prime} \rightarrow \eta\right) \\
& =p_{\eta} \sum_{\eta^{\prime}} P\left(\eta^{\prime} \rightarrow \eta\right),
\end{aligned}
$$

onde nós usamos a condição de microrreversibilidade. No entanto, a condição de normalização (4.2.1) agora nos diz que

$$
W(\eta, n+1)=p_{\eta}
$$

como queríamos demonstrar.

Segundo, nós mostramos que o desvio entre as probabilidades reais de ocorrência e a distribuição de Gibbs diminuem à medida que nós progredimos ao longo da cadeia de Markov. Nós definimos a "diferença" $D_{n}$ no passo $n$ entre a distribuição de probabilidade real dos estados e a distribuição de Gibbs:

$$
D_{n} \equiv \sum_{\eta}\left|W(\eta, n)-p_{\eta}\right| .
$$

Então,

$$
\begin{aligned}
D_{n} & =\sum_{\eta}\left|W(\eta, n)-p_{\eta}\right| \\
& =\sum_{\eta}\left|\sum_{\eta^{\prime}} W\left(\eta^{\prime}, n\right) P\left(\eta^{\prime} \rightarrow \eta\right)-p_{\eta}\right| \\
& =\sum_{\eta}\left|\sum_{\eta^{\prime}}\left[W\left(\eta^{\prime}, n\right) P\left(\eta^{\prime} \rightarrow \eta\right)-p_{\eta} P\left(\eta \rightarrow \eta^{\prime}\right)\right]\right| \\
& =\sum_{\eta}\left|\sum_{\eta^{\prime}}\left[W\left(\eta^{\prime}, n\right)-p_{\eta^{\prime}}\right] P\left(\eta^{\prime} \rightarrow \eta\right)\right|
\end{aligned}
$$


onde nós utilizamos a regra da soma (4.2.1) para ir da segunda linha para a terceira e a condição de microreversibilidade para ir da terceira linha para a quarta. Desde que $P$, sendo uma probabilidade, deve ser positiva sempre, a desigualdade triangular aplicada à (4.2.11) fornece

$$
\begin{aligned}
D_{n+1} & \leq \sum_{\eta \eta^{\prime}}\left|W\left(\eta^{\prime}, n\right)-p_{\eta^{\prime}}\right| P\left(\eta^{\prime} \rightarrow \eta\right) \\
& =\sum_{\eta^{\prime}}\left|W\left(\eta^{\prime}, n\right)-p_{\eta^{\prime}}\right| \\
& =D_{n}
\end{aligned}
$$

onde nós utilizamos novamente (4.2.1) para ir da primeira linha para a segunda. Em outras palavras, o desvio $D_{n}$ da distribuição de Gibbs decresce de forma constante ao longo de uma cadeia de Markov.

\subsubsection{Algoritmo de Metropolis}

O algoritmo mais frequentemente utilizado em processos Markovianos é aquele inventado por Metropolis e coautores [40]. A diferença na energia de um sistema devido a uma mudança na configuração $\eta$ para $\eta^{\prime}$ é calculada. Se a diferença na energia é negativa, então a nova configuração é automaticamente aceita; se, no entanto, a diferença é positiva, a nova configuração é aceita com probabilidade $e^{-\beta\left(E_{\eta^{\prime}}-E_{\eta}\right)}$. Em outras palavras,

$$
P\left(\eta \rightarrow \eta^{\prime}\right)= \begin{cases}A^{-1} & \text { se } E_{\eta^{\prime}}<E_{\eta} \\ A^{-1} e^{-\beta\left(E_{\eta^{\prime}}-E_{\eta}\right)} & \text { se } E_{\eta^{\prime}}>E_{\eta}\end{cases}
$$

De maneira estruturada, para um sistema de spins o que temos que fazer é indicao pelos cinco passos a seguir:

1. Selecionamos um sítio $i$ da rede no qual o spin $\sigma_{i}$ é considerado para inverter o sentido $\left(\sigma_{i} \rightarrow-\sigma_{i}\right)$.

2. Computamos a diferença de energia $E_{\eta^{\prime}}-E_{\eta}$ associada com a inversão.

3. Calculamos a probabilidade de transição $p=A P$ para essa inversão de acordo com $(4.2 .15)$.

4. Obtemos um número aleatório $r$ distribuído uniformemente entre zero e um.

5. Se $r<p$ invertemos o spin, do contrário não o fazemos. 
Nesse caso, a configuração de spins obtida no final do passo 5 é contada como uma "nova configuração". Na realidade, a literatura utiliza uma convenção que diz que uma "nova configuração" é considerada somente após $L^{2}$ tentativas e isso define um passo de Monte Carlo, ou no inglês, "sweep" (varredura). Nessa dissertação adotaremos o termo varredura.

A escolha da variável no passo 1 pode ser feita de diversas maneiras. Por exemplo, no caso de sistemas de spins, poderíamos escolher um spin de maneira aleatória. Nós, em contrapartida, adotamos a escolha sequencial dos spins no passo 1.

\subsubsection{Tempo de autocorrelação integrado}

Atualizações locais de Monte Carlo como o algoritmo de Metropolis não são eficientes e os dados produzidos correspondem aos sucessivos estados de uma cadeia de Markov. Esta cadeia pode fornecer configurações com observáveis altamente correlacionados, introduzindo viés nas estimativas das grandezas físicas e suas variâncias se a amostragem não for suficientemente grande. Além disso, sistemas com interações de longo alcance vão muito lentamente para o equilíbrio, o que introduz um novo grau de complexidade.

A cadeia é constituída por uma sequência de $n$ configurações as quais fornecem os observáveis $X_{i}$, com $i=1, \ldots, n_{\tau}$. Definimos a função de autocorrelação normalizada como [41]:

$$
\rho(t) \equiv \frac{C(t)}{C(0)}
$$

onde

$$
C(t) \equiv \frac{1}{n_{\tau}-|t|} \sum_{i=1}^{n_{\tau}-|t|}\left(X_{i}-\bar{X}\right)\left(X_{i+|t|}-\bar{X}\right),
$$

com $\bar{X}$ sendo a média do observável $X$ feita utilizando $n_{\tau}$ pontos da cadeia.

O tempo de autocorrelação integrado é definido como

$$
\tau_{\text {int }}=\frac{1}{2} \sum_{t=-\infty}^{\infty} \rho(t)=\frac{1}{2}+\sum_{t=1}^{\infty} \rho(t) .
$$

A variância da cadeia do observável $X$ é dada pela seguinte expressão [41]:

$$
\begin{aligned}
\sigma^{2}(\bar{X}) & =\frac{1}{n_{\tau}} \sum_{t=-\left(n_{\tau}-1\right)}^{n_{\tau}-1}\left(1-\frac{|t|}{n_{\tau}}\right) C(t) \\
& \approx \frac{1}{n_{\tau}}\left(2 \tau_{i n t}\right) C(0) \quad \text { para } n_{\tau} \gg \tau_{i n t} .
\end{aligned}
$$

O fator $2 \tau_{i n t} / n_{\tau}$ representa o erro cometido na variância devido a correlação entre os observáveis da cadeia. O fato de não considerarmos essa correlação implica na determinação de uma 
variância menor do que a variância real. Na seção 5.2.2 mostramos como as estimativas da variância, e portanto das barras de erro, são efetuadas através do método de Jackknife.

\subsection{Algoritmo multicanônico}

Desde a década de 70 é sabido que simulações de Monte Carlo com fatores de peso desconhecidos a priori eram possíveis e precisavam ser considerados [42]. Com os trabalhos de Berg e Neuhaus [43,44] essa idéia tornou-se mais amplamente aceita. Vale comentar que a motivação dos estudos de Berg e Neuhaus [43, 44] envolvia fundamentalmente o problema da barreira de energia livre (vide seção 2.3) nas transições de fase de primeira ordem. Apresentamos a seguir os aspectos envolvidos na formulação do algoritmo multicanônico. Como principais referências sobre o assunto temos as Refs. [42, 45].

O algoritmo multicanônico é caracterizado essencialmente pelo peso multicanônico $w_{\mathrm{mu}}(E)$, que é definido por:

$$
w_{\mathrm{mu}}(E) \equiv e^{-b(E) E+a(E)}
$$

onde $b(E)$ e $a(E)$ são denominados parâmetros multicanônicos. O principal efeito da utilização desse peso nas simulações de Monte Carlo é fazer com que todas as configurações sejam amostradas com a mesma frequência. Isso é pertinente principalmente para o caso onde queremos calcular o termo de energia livre de superfície em uma transição de primeira ordem [42]. As configurações são amostradas com a mesma frequência pois, da forma como é definido, o peso multicanônico $w_{\mathrm{mu}}(E)$ serve como uma aproximação para o peso $^{6}$ :

$$
w_{1 / \Omega}(E)=\frac{1}{\Omega(E)},
$$

onde $\Omega(E)$ é a degenerescência dos estados com energia ${ }^{7} E$.

Na prática, o algoritmo multicanônico é implementado de maneira análoga ao algoritmo de Metropolis apresentado na seção 4.2.1. As configurações, no entanto, devem ser amostradas de acordo com uma distribuição definida utilizando o peso multicanônico $w_{\mathrm{mu}}\left(E_{\eta}\right)$. Isso é feito considerando a probabilidade de transição como:

$$
P_{\mathrm{mu}}\left(\eta \rightarrow \eta^{\prime}\right)= \begin{cases}A_{\mathrm{mu}}^{-1} & \text { se } E_{\eta^{\prime}}<E_{\eta} \\ A_{\mathrm{mu}}^{-1} w_{\mathrm{mu}}\left(\eta^{\prime}\right) / w_{\mathrm{mu}}(\eta) & \text { se } E_{\eta^{\prime}}>E_{\eta}\end{cases}
$$

\footnotetext{
${ }^{6}$ Outro algoritmo que também explora esse mesmo princípio é o algoritmo de Wang-Landau, proposto na Ref. [46].

${ }^{7}$ Um algoritmo semelhante foi proposto por Lee [47], o qual define o peso associando-o com a entropia microcanônica. Considera $\Omega(E) \equiv e^{S(E) / k_{B}}$, o que leva à analogia $S(E) \approx b(E) E-a(E)$.
} 
onde $w_{\mathrm{mu}}(\eta)$ é o peso multicanônico definido de acordo com a expressão (4.3.1) para a energia $E_{\eta}$. Note que aqui devemos associar índices inteiros a cada uma das energias, fazemos isso de acordo com o discutido na seção 4.1 .

Seguindo o algoritmo definido em (4.3.3), obtemos uma distribuição de configurações (i.e., um histograma da série produzida) que corresponderá à probabilidade multicanônica, a qual, por sua vez, é dada por

$$
p_{\mathrm{mu}}(E)=c_{\mathrm{mu}} \Omega(E) w_{\mathrm{mu}}(E) .
$$

Podemos utilizar a aproximação

$$
\Omega(E) \approx\left[w_{\mathrm{mu}}(E)\right]^{-1}
$$

para reescrever a probabilidade $p_{\mathrm{mu}}(E)$ como

$$
p_{\mathrm{mu}}(E) \approx c_{\mathrm{mu}}
$$

a qual fornece um histograma aproximadamente plano.

Podemos também obter a probabilidade canônica de encontramos o sistema com uma energia $E$ a uma temperatura $T=\left(k_{B} \beta\right)^{-1}$, definida como

$$
p_{B}(E, \beta)=\Omega(E) e^{-\beta E} / \mathcal{Z}(\beta)
$$

utilizando os pesos multicanônicos. Fazemos isso considerando a aproximação (4.3.5), ou seja, escrevemos a probabilidade canônica como

$$
p_{B}(E, \beta) \approx\left[w_{\mathrm{mu}}(E)\right]^{-1} e^{-\beta E} / \mathcal{Z}(\beta) .
$$

O problema é que os pesos $w_{\mathrm{mu}}(E)$ não são conhecidos a priori. Assim, precisamos dividir a implementação do algoritmo multicanônico em dois passos principais:

(i) obtenção dos parâmetros multicanônicos $a(E)$ e $b(E)$, os quais definem os pesos multicanônicos $w_{\mathrm{mu}}(E)$;

(ii) produção de séries de dados utilizando os parâmetros multicanônicos $a(E)$ e $b(E)$ determinados no passo (i).

O passo (ii) é trivial já que é executado de maneira completamente análoga ao algoritmo de Metropolis, como definido em (4.3.3). Para o primeiro passo porém, vamos considerar aqui o método das relações recursivas, o qual é amplamente discutido nas Refs. [42, 45] e consiste em realizar diversas simulações para a determinação dos parâmetros multicanônicos $a(E)$ e $b(E)$. 
Primeiro consideramos os pesos

$$
w^{0}(\eta)=w^{0}\left(E_{\eta}\right)=1 \quad \text { para todo } \eta
$$

e as simulações seguem tentando obter melhores estimativas para $w^{n}(\eta),(n=1,2,3, \ldots)$. A condição inicial acima corresponde a uma situação de temperatura infinita onde os $a^{0}(E)$ e $b^{0}(E)$ são identicamente nulos ${ }^{8}$ seja qual for o valor de $E$.

A $n$-ésima simulação é efetuada com o peso $w^{n}(\eta)$ e fornece um histograma $H_{\mathrm{mu}}^{n}\left(E_{\eta}\right)$, o qual é utilizado para definir o peso $w^{n+1}(\eta)$.

Consideramos agora a analogia

$$
S(E)=b(E) E-a(E)
$$

onde $S(E)$ é a entropia microcanônica, vamos estabelecer a seguir as relações recursivas entre os parâmetros multicanônicos.

Por analogia à relação termodinâmica $1 / T=(\partial S / \partial E)$, definimos a equação

$$
b(E)=\frac{\partial S(E)}{\partial E}
$$

a qual pode ser reescrita para energias $\operatorname{discretas}^{9}$ da seguinte maneira:

$$
b(E)=\frac{S(E-\epsilon)-S(E)}{\epsilon} .
$$

Utilizando a identidade (4.3.10), calculamos a diferença $S(E)-S(E-\epsilon)$ :

$$
S(E)-S(E-\epsilon)=b(E) E-b(E-\epsilon)(E-\epsilon)-a(E)+a(E-\epsilon) .
$$

Agora, inserindo $\epsilon b(E-\epsilon)=S(E)-S(E-\epsilon)$ via equação acima, obtemos

$$
a(E-\epsilon)=a(E)+[b(E-\epsilon)-b(E)] E .
$$

Assim, definindo um valor arbitrário para $a\left(E_{M}\right)$ (por exemplo $a\left(E_{M}\right)=0$ ), conseguimos atribuir valores para $a(E)$ para qualquer $E$ através da expressão acima. Desta forma vemos que, se $b(E)$ for dado para todos os valores de $E$, obtemos todos os valores de $a(E)$. Notamos que os valores de $a^{n}(E)$ dependem apenas dos valores de $b^{n}(E)$ na $n$-ésima simulação. Lembrando que a condição (4.3.9) define os valores iniciais.

\footnotetext{
${ }^{8}$ É aconselhável começar a recorrência $(n=0)$ para os pesos multicanônicos na região desordenada, $b^{0}(E)=$ 0 e $a^{0}(E)=0$, pois assim o sistema move-se livremente nesta fase, embora a escolha $b^{0}(E)=\beta$ também seja possível.

${ }^{9}$ Aqui utilizamos também a discretização descrita pela expressão (4.1.6).
} 
Precisamos ainda de uma relação que atribua os valores para $b^{n+1}(E)$ a partir dos valores de $b^{n}(E)$ na $n$-ésima simulação. Obtemos essas relação utilizando o histograma $H_{m u}^{n}(E)$ obtido na $n$-ésima simulação.

Para evitar algum valor nulo de $H_{m u}^{n}(E)$, substituimos $H_{m u}^{n}(E)$ por

$$
\hat{H}_{m u}^{n}(E)=\max \left[h_{0}, H_{m u}^{n}(E)\right]
$$

onde $0<h_{0}<1$.

Definimos então

$$
w^{n+1}(k)=e^{-S^{n+1}(E)}=c \frac{w^{n}(k)}{\hat{H}_{\mathrm{mu}}^{n}\left(E_{k}\right)},
$$

onde a constante (irrelevante) c é introduzida para que $S^{n+1}(E)$ possa ser um estimador da entropia microcanônica:

$$
S^{n+1}(E)=-\ln c+S^{n}(E)+\ln \hat{H}_{\mathrm{mu}}^{n}(E)
$$

Substituindo essa expressão na equação (4.3.12) obtemos a seguinte relação para os $b(E)$ :

$$
b^{n+1}(E)=b^{n}(E)+\left[\ln \hat{H}_{\mathrm{mu}}^{n}(E+\epsilon)-\ln \hat{H}_{\mathrm{mu}}^{n}(E)\right] / \epsilon .
$$

Na realidade utilizamos a relação descrita nas Refs. [42, 45], onde define-se o peso normalizado

$$
\hat{g}_{0}^{n}(E)=\frac{g_{0}^{n}(E)}{g^{n}(E)+g_{0}^{n}(E)} .
$$

Os pesos $g^{n}(E)$ são determinados pela relação recursiva

$$
g^{n+1}(E)=g^{n}(E)+g_{0}^{n}(E)
$$

$\operatorname{com} g^{0}(E)=0$. Os pesos $g_{0}^{n}(E)$ são determinados a partir dos histogramas através da relação

$$
g_{0}^{n}(E)=\frac{\hat{H}_{\mathrm{mu}}^{n}(E+\epsilon) \hat{H}_{\mathrm{mu}}^{n}(E)}{\hat{H}_{\mathrm{mu}}^{n}(E+\epsilon)+\hat{H}_{\mathrm{mu}}^{n}(E)} .
$$

Com isso, a relação recursiva para $b(E)$ é reescrita utilizando $\hat{g}_{0}^{n}(E)$ como

$$
b^{n+1}(E)=b^{n}(E)+\hat{g}_{0}^{n}(E) \times\left[\ln \hat{H}_{\mathrm{mu}}^{n}(E+\epsilon)-\ln \hat{H}_{\mathrm{mu}}^{n}(E)\right] / \epsilon
$$




\section{Capítulo 5}

\section{Simulação do modelo de Ising dipolar bidimensional}

Neste capítulo apresentamos o estudo realizado sobre o modelo de Ising dipolar bidimensional utilizando simulações numéricas de Monte Carlo. Além de discutir a implementação das condições de contorno periódicas, apresentamos os aspectos importantes da análise de dados tais como a técnica de repesagem em histogramas múltiplos e a estimativa de barras de erro pelo método de Jackknife.

Apresentamos também os resultados obtidos por meio dos algoritmos de Metropolis e multicanônico para diversos tamanhos de rede. A partir dos nossos resultados foi possível identificar as fases termodinâmicas do modelo para $\delta=2$. Para tamanhos de rede menores $(L=16$ e 32$)$, mostramos que essas fases correspondem às fases de líquido tetragonal e de faixas de spins com largura $h=2$. Para tamanhos de redes maiores $(L=48,56$ e 72), encontramos evidências numéricas da existência de uma fase intermediária, a fase nemática de Ising, a qual foi identificada recentemente por Cannas e coautores [33] por meio de simulações de Monte Carlo. Neste trabalho calculamos o calor específico e a susceptibilidade do parâmetro de ordem no intervalo de temperaturas onde as transições de fase são observadas. Para esta finalidade, implementamos a técnica de repesagem em histogramas múltiplos. Com esta metodologia identificamos os máximos dessas grandezas e estimamos as temperaturas críticas de tamanho finito com grande precisão.

Com os valores dos máximos do calor específico, obtidos através da repesagem das séries temporais produzidas por meio do algoritmo de Metropolis, realizamos uma análise de tamanho finito e obtivemos os expoentes críticos $\alpha, \gamma$ e $\nu$, e discutimos a natureza das transições de fase. Por fim, calculamos o tempo de autocorrelação integrado para as séries da energia obtidas por meio do algoritmo de Metropolis em diversas temperaturas. A partir dos resultados obtidos para temperaturas próximas às temperaturas de transição de fase (para as transições 
que ocorrem nos tamanhos de rede maiores), estimamos o expoente crítico dinâmico ${ }^{1} z_{\text {int }}$. Inferimos daí o quão severo é o decaimento lento crítico para esse sistema próximo às transições de fase termodinâmicas.

Vale notar que o estudo realizado aqui sobre o modelo de Ising dipolar, o qual inclui:

i. o uso da técnica de repesagem para determinação das grandezas físicas,

ii. o cálculo do tempo de autocorrelação nas séries da energia para diferentes temperaturas,

iii. o cálculo do expoente crítico dinâmico,

vi. estimativas para os expoentes críticos $\alpha, \gamma$ e $\nu$ para as transições de fase faixas-nemática e nemática-tetragonal,

v. a utilização de um algoritmo de minimização global (multicanônico), ainda não foi abordado na literatura ${ }^{2}$.

Ressaltamos ainda que, para realizar as simulações e a análise dos dados obtidos, foi necessário desenvolver diversos códigos computacionais. Inclui-se aqui os códigos da implementação para:

i. condições de contorno periódicas considerando o termo dipolar, onde empregamos a técnica do somatório de Ewald. Os aspectos analíticos, a implementação e um estudo numérico da convergência dessa técnica estão contidos no Apêndice A,

ii. produção das séries temporais para a energia e parâmetro de ordem $O_{h v}$ por meio do algoritmo de Metropolis,

iii. geração de histogramas a partir das séries obtidas,

vi. armazenamento de configurações dos spins,

v. análise das séries obtidas pelo algoritmo multicanônico, a qual incorpora, além das estimativas de erro utilizando o método de Jackknife, a técnica de repesagem empregando o método de soma logaritmica [45].

vi. atualização dos parâmetros multicanônicos $a(E)$ e $b(E)$,

\footnotetext{
${ }^{1}$ Vide Ref. [41] para um melhor detalhamento.

${ }^{2}$ Exceto pelos resultados obtidos por meio do algoritmo multicanônico, todos os resultados obtidos por nós, juntamente com as discussões realizadas, já foram redigidos na forma de artigo científico e submetidos para publicação. O texto pode ser encontrado no site http://arxiv.org/abs/0903.4084.
} 
vii. produção das séries por meio do algoritmo multicanônico,

viii. análise das séries obtidas pelo algoritmo de Metropolis, a qual incorpora a técnica de repesagem em histogramas múltiplos e as estimativas de erro utilizando o método de Jackknife,

\subsection{Condições de contorno periódicas}

Condições de contorno periódicas são amplamente utilizadas em simulações numéricas. Tanto métodos de Monte Carlo quanto métodos de dinâmica molecular as utilizam. Sua implementação é fundamental para amenizar efeitos do tamanho finito e eventualmente eliminar efeitos de borda [48, 49]. Além disso, transições que são conhecidas como de primeira ordem frequentemente exibem características de transições de ordem superior quando modeladas em caixas pequenas por causa da supressão das flutuações.

Outro detalhe importante sobre a implementação das condições de contorno, em específico no modelo de Ising dipolar, está relacionado com a formação das estrutura de domínios do tipo faixas para o estado fundamental. O comprimento $L$ deve ser múltiplo de $2 h$ para que o sistema possa minimizar a energia sem que os efeitos de borda causados pelas condições de contorno sejam significativos.

A Figura 5.1 mostra uma representação gráfica de como as condições de contorno periódicas são definidas. O sistema, representado pela caixa central $O$ com lados iguais a $L$, tem sua imagem (caixas com linhas pontilhadas) replicada infinitamente nas direções dos versores $\hat{e}_{1}$ e $\hat{e}_{2}$ no plano $x y$, formando uma rede de Bravais regular bidimensional ${ }^{3}$. Utilizamos o vetor $\vec{n}=L\left(n_{1} \hat{e}_{1}+n_{2} \hat{e}_{2}\right)$ para indicar a posição de cada uma das imagens replicadas do sistema, com $n_{1}$ e $n_{2}$ sendo números inteiros (positivos e negativos). Desse modo, quando $\vec{n}=0$, estamos nos referindo ao próprio sistema e, quando $\vec{n} \neq 0$, estamos nos referindo às suas imagens. Como o modelo apresenta dois termos de interação, é conveniente implementar as condições de contorno periódicas em duas partes: uma para as interações entre os primeiros vizinhos e outra para as interações dipolares.

As condições de contorno periódicas considerando apenas as interações entre os primeiros vizinhos está representada pelas linhas vermelhas pontilhadas. Note que os primeiros vizinhos da partícula $i$ não estão situados apenas na caixa central $\mathrm{O}$, mas distam apenas de uma unidade de espaçamento de rede em relação a essa partícula. Portanto, para a interação de troca, nota-

\footnotetext{
${ }^{3} \mathrm{~A}$ título de clareza apenas oito imagens (A-H) do sistema estão presentes na Figura 5.1, contudo, temos infinitas imagens replicadas.
} 


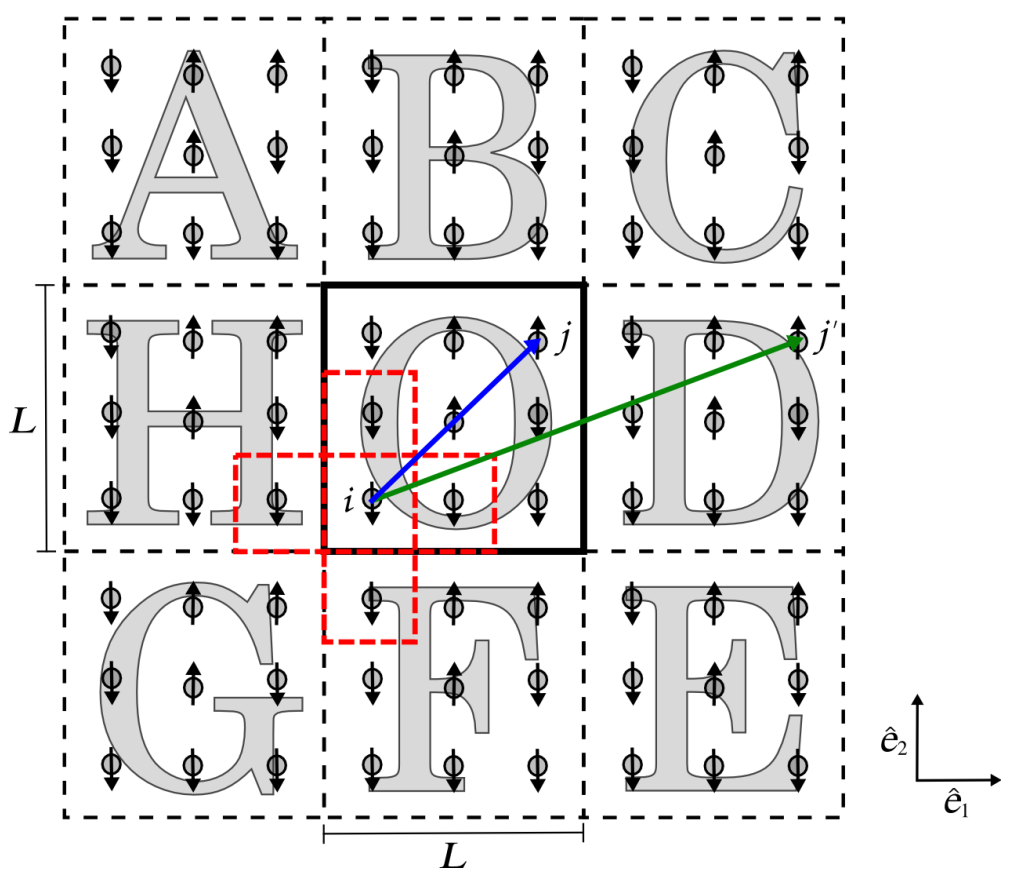

Figura 5.1: Representação das condições de contorno periódicas para interações entre primeiros vizinhos (linhas vermelhas pontilhadas) e para interações dipolares (vetores coloridos).

se que é suficiente replicar a última coluna (à direita) da caixa $\mathrm{O}$ ao lado da primeira coluna (à esquerda) e vice-versa; e, de modo análogo, replicar a última linha da caixa $\mathrm{O}$ acima da primeira linha e vice-versa.

A exigência de imagens replicadas infinitamente no plano vem do carácter de longo alcance da interação dipolar. O momento de dipolo magnético do spin da partícula $i$ deve interagir com todos os momentos de dipolo dos spins das outras partículas e também das suas respectivas imagens, incluindo as interações com os momentos de dipolo dos spins das suas próprias imagens. Por exemplo, na Figura 5.1, o vetor azul representa o vetor $\vec{R}_{i j}$, cujo o módulo é utilizado para o cálculo da interação entre os momentos de dipolo correspondentes aos spins das partículas $i$ e $j$. A interação entre o momento de dipolo do spin da partícula $i$ com o momento de dipolo da imagem da partícula $j$ é obtida utilizando o módulo do vetor verde $\vec{R}_{i j^{\prime}}$, que é dado pela soma $\vec{n}+\vec{R}_{i j}$. Neste caso temos $n_{1}=1$ e $n_{2}=0$, isto é, $\vec{n}=L \hat{e}_{1}$, o que indica que a imagem correspondente do sistema é representada pela caixa $D$.

Portanto, incluir a interação dos momentos de dipolo dos spins das partículas do sistema com suas imagens equivale a somar ao vetor ${ }^{4} \vec{r}_{i j}$ o vetor $\vec{n}$, efetuando somas infinitas sobre as componentes do vetor $\vec{n}$. Assim, podemos reescrever o termo de interação dipolar da energia

\footnotetext{
${ }^{4}$ Lembramos que $\vec{R}_{i j}=a \vec{r}_{i j}$, onde $a$ é o espaçamento de rede, e como é usual, fazemos $a=1$. Isso implica que o volume do sistema é $L^{2}=N$, onde $N$ é o número total de spins.
} 
em (3.1.8) como

$$
E_{\text {dipolar }}=\frac{1}{2} \sum_{i=1}^{N} \sum_{j=1}^{N} \sum_{|\vec{n}|=0}^{\infty}{ }^{\prime} \frac{\sigma_{i} \sigma_{j}}{\left|\vec{r}_{i j}+\vec{n}\right|^{3}},
$$

onde o símbolo ' indica que o vetor $\vec{n}=0$ é excluído do somatório quando $i=j$, impedindo a auto-interação. O fator $1 / 2$ aparece pois a energia de interação entre as partículas $i$ e $j$ só deve ser computada uma vez.

Uma forma eficiente para calcular $E_{\text {dipolar }}$ é por meio do método do somatório de Ewald, apresentado em detalhes no Apêndice A. Utilizando este método, convertemos o somatório na rede ${ }^{5}$, o qual converge lentamente, em dois outros somatórios, sendo um no espaço real e o outro no espaço recíproco. Estes novos somatórios possuem uma melhor convergência, ou seja, utilizam um menor número de termos na soma para convergirem. A aplicação do método de Ewald leva ainda à existência de um terceiro termo, chamado auto-interação. Assim podemos reescrever a expressão (5.1.1) da seguinte maneira:

$$
E_{\text {dipolar }}=E_{\text {real }}+E_{\text {recíproco }}+E_{\text {auto-interação }}
$$

onde

$$
\begin{aligned}
& E_{\text {real }}=\frac{1}{2} \sum_{i=1}^{N} \sum_{j=1}^{N} \sum_{|\vec{n}|=0}^{\infty}{ }^{\prime} \sigma_{i} \sigma_{j}\left[\frac{\operatorname{erfc}\left(\alpha\left|\vec{r}_{i j}+\vec{n}\right|\right)}{\left|\vec{r}_{i j}+\vec{n}\right|^{3}}+\frac{2 \alpha}{\sqrt{\pi}} \frac{e^{-\alpha^{2}\left|\vec{r}_{i j}+\vec{n}\right|^{2}}}{\left|\vec{r}_{i j}+\vec{n}\right|^{2}}\right] \\
& E_{\text {recíproco }}=\frac{\pi}{L^{2}} \sum_{i=1}^{N} \sum_{j=1}^{N} \sum_{|\vec{G}|=0}^{\infty} \sigma_{i} \sigma_{j}\left[\frac{2 \alpha}{\sqrt{\pi}} e^{-\frac{G^{2}}{4 \alpha^{2}}}-G \operatorname{erfc}\left(\frac{G}{2 \alpha}\right)\right] \cos \left(\vec{G} \cdot \vec{r}_{i j}\right), \\
& E_{\text {auto-interação }}=-\frac{2 \alpha^{3}}{3 \sqrt{\pi}} \sum_{i=1}^{N} \sigma_{i}^{2}
\end{aligned}
$$

$\operatorname{com} \vec{G}=2 \pi\left(k_{1} \hat{e}_{1}+k_{2} \hat{e}_{2}\right) / L$, e sendo $k_{1}$ e $k_{2}$ números inteiros (positivos e negativos). Nós definimos o parâmetro $\alpha$ de Ewald igual a 3,5 em todas as simulações. A justificativa desse valor vem do nosso estudo numérico da convergência dos somatórios, o qual é apresentado na seção A.4 do Apêndice. Também apresentamos, na seção A.3, uma maneira de otimizar o cálculo dos somatórios. Para isso, utilizamos matrizes para o armazenamento de parte dos somatórios das expressões (5.1.3a) e (5.1.3b).

\footnotetext{
${ }^{5}$ Especificamente sobre as componentes do vetor $\vec{n}$.
} 


\subsection{Metodologias para análise de dados}

\subsubsection{Técnica de repesagem}

Como vimos, as simulações de Monte Carlo geram cadeias com valores para o observável $X$ segundo uma distribuição de probabilidades definida. Em Mecânica Estatística, estamos interessados em utilizar essas cadeias para obter estimadores $\bar{X}(\beta)$ e $\bar{X}^{2}(\beta)$ para médias térmicas $\langle X\rangle(\beta)$ e $\left\langle X^{2}\right\rangle(\beta)$ desse observável, respectivamente.

No algoritmo de Metropolis as cadeias são geradas segundo o peso $w_{k}=e^{-\beta_{0} E_{k}}$, onde $\beta_{0}=$ $1 / k_{B} T_{0}$. Assim, em princípio, poderíamos apenas estimar as médias térmicas na "temperatura" $\beta_{0}$ e para temperaturas diferentes dessa, teríamos que realizar outras simulações. Para o algoritmo multicanônico o caso parece ser pior, já que as cadeias são geradas de acordo com um peso $w_{k}=w_{\mathrm{mu}}\left(E_{k}\right)$ e (ainda) não temos nenhuma relação que possamos associar com a temperatura canônica. Nesses casos, a técnica de repesagem é extremamente útil, já que permite utilizar os dados obtidos nessas cadeias para fornecer médias térmicas para valores contínuos da "temperatura" $\beta[50,51,52]$.

De uma maneira geral, realizamos a repesagem de uma cadeia com $n$ valores $X_{1}, \ldots, X_{n}$ para o observável $X$ de acordo com a distribuição canônica de Gibbs. Assim, um estimador adequado para a média térmica da grandeza $\langle X\rangle$ para uma dada "temperatura" $\beta$ é dado por

$$
\bar{X}(\beta)=\frac{1}{\bar{Z}(\beta)} \sum_{k=1}^{n} X_{k} w_{k}^{-1} e^{-\beta E_{k}},
$$

onde

$$
\bar{Z}(\beta)=\sum_{k=1}^{n} w_{k}^{-1} e^{-\beta E_{k}}
$$

equivale a um estimador da função de partição canônica, sendo $w_{k}$ o peso utilizado para amostrar a série original. Note que para cada valor $X_{k}$ do observável $X$ fornecido pela cadeia temos associado uma energia $E_{k}$.

No algoritmo multicanônico temos que $w_{k}=e^{-b\left(E_{k}\right) E_{k}+a\left(E_{k}\right)}$, assim a repesagem é feita da seguinte maneira:

$$
\bar{X}(\beta)=\frac{1}{\bar{Z}(\beta)} \sum_{k=1}^{n} X_{k} e^{-\beta E_{k}+b\left(E_{k}\right) E_{k}-a\left(E_{k}\right)},
$$

onde

$$
\bar{Z}(\beta)=\sum_{k=1}^{n} e^{-\beta E_{k}+b\left(E_{k}\right) E_{k}-a\left(E_{k}\right)} .
$$


É importante comentar que os números que vão dentro da exponencial são, em geral, muito grandes, o que leva a sérios problemas numéricos. Esses problemas podem ser amenizados pelo método de soma proposto na Ref. [45], o qual utiliza funções logaritmicas.

Como já mencionado, para o algoritmo de Metropolis temos que $w_{k}=e^{-\beta_{0} E_{k}}$. Podemos repesar as séries obtidas por meio do algoritmo de Metropolis, utilizando o peso $w_{k}$, em uma "temperatura" $\beta$ da seguinte maneira:

$$
\bar{X}(\beta)=\frac{1}{\bar{Z}(\beta)} \sum_{k=1}^{n} X_{k} e^{-\left(\beta_{0}-\beta\right) E_{k}},
$$

onde

$$
\bar{Z}(\beta)=\sum_{k=1}^{n} e^{-\left(\beta_{0}-\beta\right) E_{k}} .
$$

Para aumentar a estatística para a repesagem final, é útil combinar os dados obtidos em simulações independentes realizadas em "temperaturas" $\beta_{0}^{i}(i=1, \ldots, P)$, com a condição de que as $P$ simulações foram realizadas para temperaturas muito próximas umas das outras. Isso leva à seguinte abordagem direta, a qual foi proposta na Ref. [50]. A primeira observação é que qualquer combinação linear ponderada,

$$
\bar{X}(\beta)=\frac{\sum_{i=1}^{P} a_{i} \bar{X}_{i}(\beta)}{\sum_{i=1}^{P} a_{i} \bar{Z}_{i}(\beta)},
$$

com os fatores de peso $a_{i}=a_{i}(\beta)>0$, é um estimador válido para $\langle X\rangle(\beta)$.

Aqui temos:

$$
\bar{X}_{i}(\beta)=\sum_{k=1}^{n} X_{k} e^{-\left(\beta-\beta_{0}^{i}\right) E_{k}}
$$

e

$$
\bar{Z}_{i}(\beta)=\sum_{k=1}^{n} e^{-\left(\beta-\beta_{0}^{i}\right) E_{k}} .
$$

Para a estimativa final na equação (5.2.7) é conveniente impor a normalização

$$
\sum_{i=1}^{P} c_{i}=1
$$

$\operatorname{com} c_{i}=a_{i} \bar{Z}_{i}$. Isso converte a equação $(5.2 .7) \mathrm{em}$

$$
\bar{X}(\beta)=\sum_{i=1}^{P} c_{i} \bar{x}_{i}
$$

$\operatorname{com} \bar{x}_{i}=\bar{X}_{i} / \bar{Z}_{i}$ e $c_{i}=c_{i}(\beta)$ sendo pesos normalizados. Estes pesos são escolhidos como sendo o inverso da variância estatística $c_{i}(\beta) \approx 1 / \sigma^{2}\left[\bar{X}_{i}(\beta)\right]$ de cada série temporal repesada. A constante geral total é determinada pela condição de normalização (5.2.10). 


\subsubsection{O método de Jackknife}

Como discutido na seção 4.2.2, os dados obtidos para as séries temporais através do algoritmo de Metropolis podem estar correlacionados. Isso implica em uma variância $\sigma^{2}(\bar{X})$ equivocada. Apresentamos aqui a estimativa correta da variância, a qual também é utilizada para o agrupamento de várias estatísticas na repesagem de cadeias obtidas através do algoritmo de Metropolis, como mencionado na seção 5.2.1. Mostramos aqui o método ${ }^{6}$ detalhado para obter as estimativas corretas das barras de erro das médias térmicas das grandezas físicas já incluindo a técnica de repesagem.

Em primeiro lugar, dividimos o número total $n$ de pontos da série temporal em $N_{A}$ amostras com $n_{A}$ pontos cada uma (do inglês: "binning"). Calculamos então $N_{A}$ médias parciais utilizando $n-n_{A}$ pontos da série, excluindo sempre $n_{A}$ pontos de uma amostra, ou seja, calculamos

$$
\begin{aligned}
\bar{X}_{j}(\beta) & =\sum_{k \notin\{j\}} X_{k} w_{k}^{-1} e^{-\beta E_{k}}, \\
\bar{X}^{2}{ }_{j}(\beta) & =\sum_{k \notin\{j\}} X_{k}^{2} w_{k}^{-1} e^{-\beta E_{k}} \\
\bar{Z}_{j}(\beta) & =\sum_{k \notin\{j\}} w_{k}^{-1} e^{-\beta E_{k}} .
\end{aligned}
$$

A notação $k \notin\{j\}$ significa que todos os pontos $n_{A}$ da $j$-ésima amostra serão excluídos do $j$-ésimo estimador, com $j=1, \ldots, N_{A}$.

Utilizando os $\bar{Z}_{j}\left(\beta_{i}\right)$, normalizamos as medidas de $\bar{X}_{j}(\beta)$ e $\bar{X}^{2}{ }_{j}(\beta)$ da seguinte maneira:

$$
\begin{aligned}
\bar{x}_{j}(\beta) & =\frac{\bar{X}_{j}(\beta)}{\bar{Z}_{j}(\beta)}, \\
\bar{x}^{2}{ }_{j}(\beta) & =\frac{\bar{X}^{2}{ }_{j}(\beta)}{\bar{Z}_{j}(\beta)} .
\end{aligned}
$$

Estes resultados permitem calcular a susceptibilidade como

$$
\bar{\chi}_{j}(\beta)=D\left[\bar{x}^{2}{ }_{j}(\beta)-\left[\bar{x}_{j}(\beta)\right]^{2}\right],
$$

onde $D$ é igual a $k_{B} \beta^{2} / N$ para o calor específico $C_{v}$ (segundo a expressão (4.1.16)) e igual a $N$ para o parâmetro de ordem $O_{h v}$ (segundo a expressão (4.1.17)).

Por fim calculamos as médias finais do observável físico e da susceptibilidade respectivamente como:

$$
\bar{x}(\beta)=\frac{1}{N_{A}} \sum_{j=1}^{N_{A}} \bar{x}_{j}(\beta)
$$

\footnotetext{
${ }^{6}$ Para um melhor detalhamento sobre o método de Jackknife vide Ref. [53].
} 
e

$$
\bar{\chi}(\beta)=\frac{1}{N_{A}} \sum_{j=1}^{N_{A}} \bar{\chi}_{j}(\beta) .
$$

Os respectivos desvios padrão são dados por:

$$
\sigma_{\bar{x}}^{2}(\beta)=\frac{\left(N_{A}-1\right)}{N_{A}} \sum_{j=1}^{N_{A}}\left[\bar{x}_{j}(\beta)-\bar{x}(\beta)\right]^{2}
$$

$\mathrm{e}$

$$
\sigma_{\bar{\chi}}^{2}(\beta)=\frac{\left(N_{A}-1\right)}{N_{A}} \sum_{j=1}^{N_{A}}\left[\bar{\chi}_{j}(\beta)-\bar{\chi}(\beta)\right]^{2} .
$$

Por exemplo, os estimadores para $\langle E\rangle(\beta)$ e $\left\langle O_{h v}\right\rangle(\beta)$ são calculados através da expressão (5.2.18) e suas respectivas barras de erro são dadas pela raíz quadrada de $\sigma_{\bar{x}}^{2}(\beta)$, conforme a equação (5.2.20). Já os estimadores para o calor específico $C_{v}(\beta)$ e a susceptibilidade $\chi_{h v}(\beta)$ do parâmetro de ordem, podem ser calculados utilizando a equação (5.2.19), com os seus respectivos erros dados pela raíz quadrada do desvio padrão $\sigma_{\bar{\chi}}^{2}(\beta)$, conforme a expressão $(5.2 .21)$.

\subsection{Resultados}

Todo o estudo apresentado aqui foi focado na região do diagrama de fase onde a razão entre as intensidades dos acoplamento $\delta$ é igual a 2. A escolha desse valor foi baseada principalmente em dois fatores: i) a possível presença da fase nemática de Ising, que, como veremos, foi confirmada pelos nossos resultados, ii) ter a largura da faixas $h=2$, visto que as simulações são bastante demoradas e, por isso, tivemos que restringir os tamanhos das redes utilizadas. Dividimos os resultados em duas seções, uma para os resultados obtidos através do algoritmo de Metropolis e outra para os obtidos através do algoritmo multicanônico. Para os dois algoritmos foram geradas séries temporais para a energia interna total por spin $E / N$ e para o parâmetro de ordem $O_{h v}$.

\subsubsection{Algoritmo de Metropolis}

Utilizando o algoritmo de Metropolis foram geradas séries temporais para redes de tamanho $L=16,32,48,56$ e 72. Na Tabela 5.1, apresentamos a lista das temperaturas $T_{0}$ utilizadas em cada produção para os tamanho de rede $L$. Todas as séries produzidas foram inicializadas com configurações aleatórias. A termalização consistiu de $10^{6}$ varreduras para tamanhos de rede $L \leq 48$ e $5 \times 10^{5}$ varreduras para $L=56$ e 72 . Para cada temperatura, as séries obtidas 
correspondem a $3,4 \times 10^{7}$ varreduras para $L \leq 56$ e $2,7 \times 10^{7}$ varreduras para o maior tamanho de rede $L=72$.
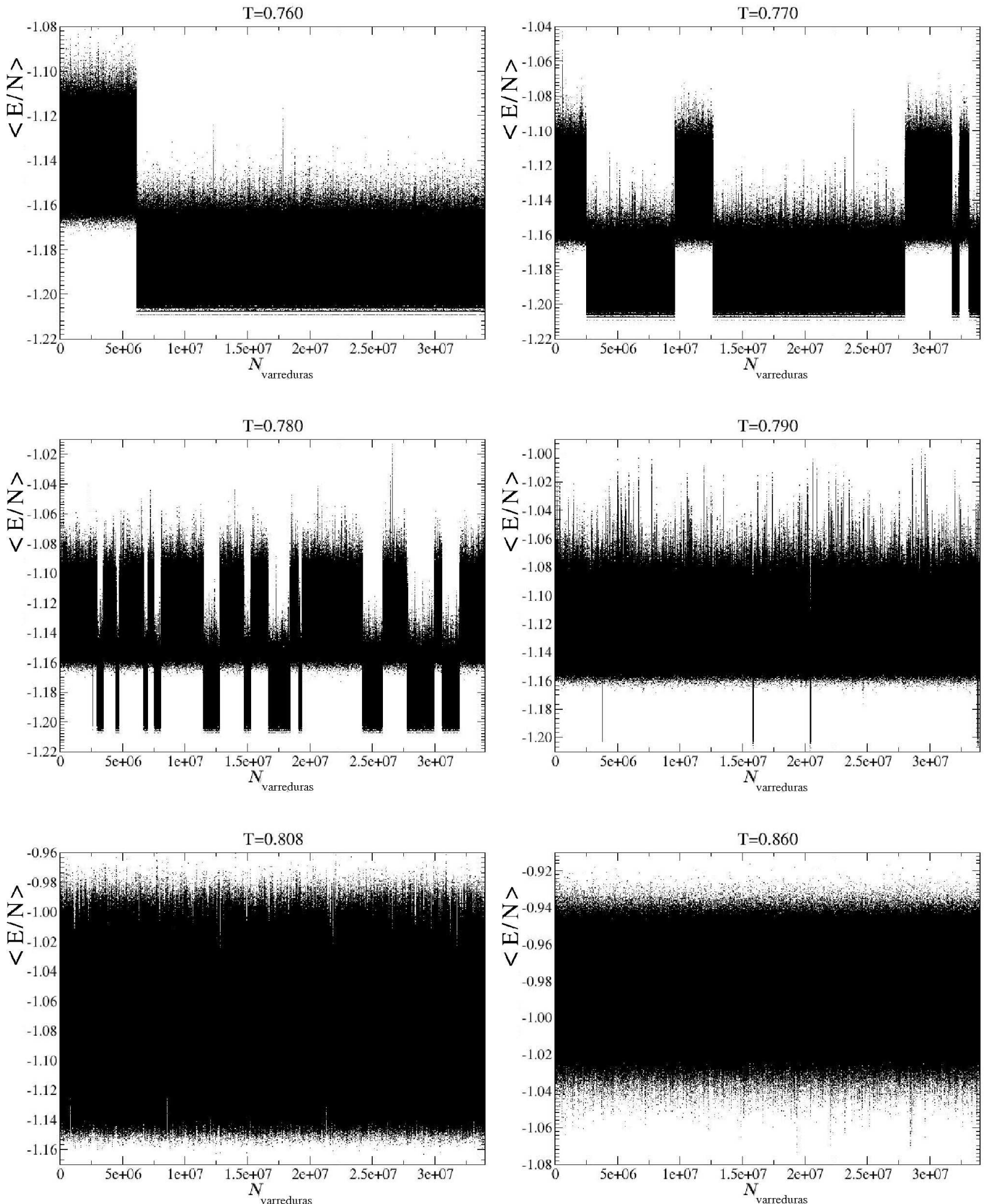

Figura 5.2: Séries temporais para a energia por spin $\langle E / N\rangle$ geradas pelo algoritmo de Metropolis para $L=56$ em diferentes temperaturas. 

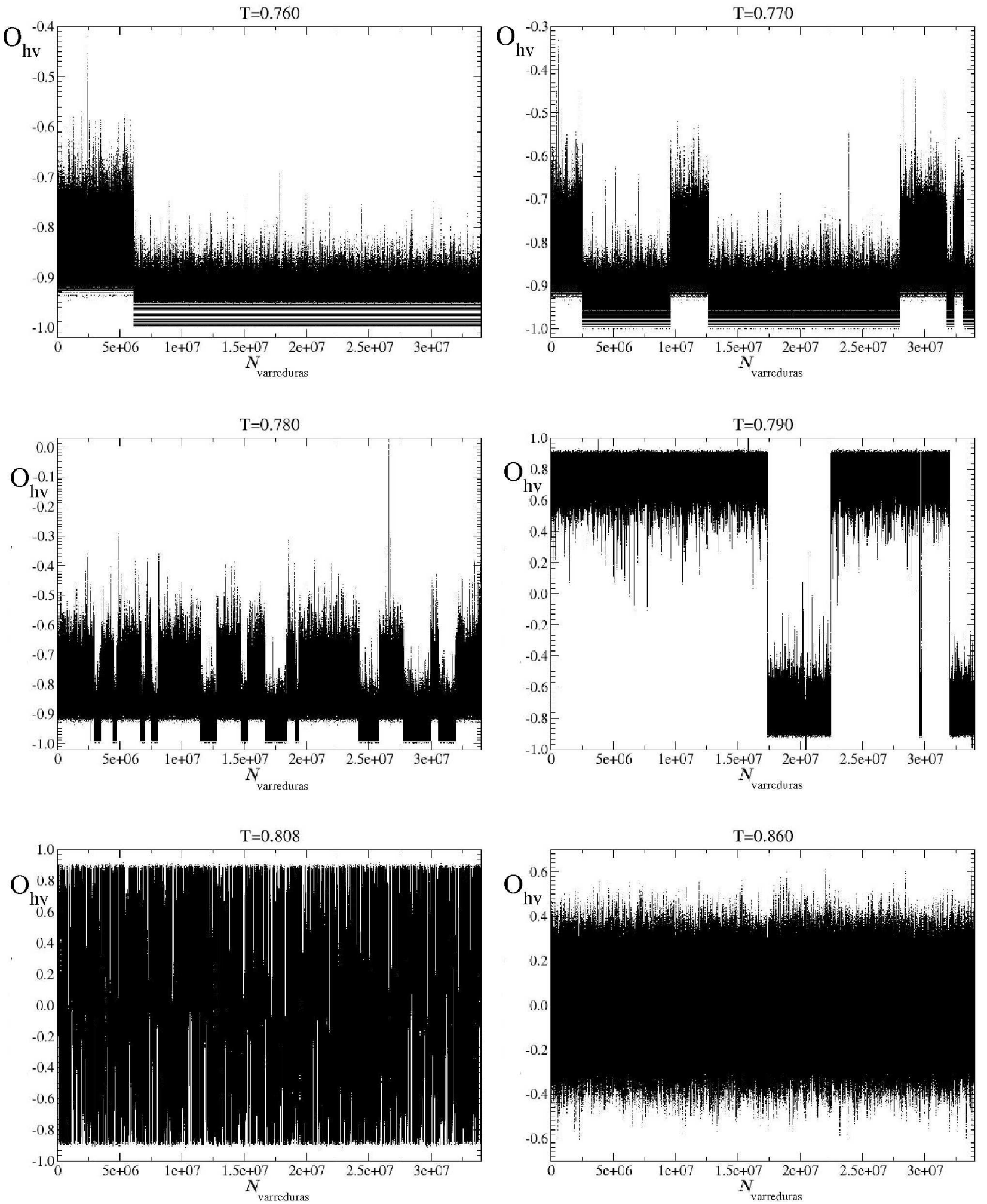

Figura 5.3: Séries temporais para o parâmetro de ordem $O_{h v}$ (sem módulo) geradas pelo algoritmo de Metropolis para $L=56$ em diferentes temperaturas.

As Figuras 5.2 e 5.3 mostram, respectivamente, as séries temporais produzidas para a energia por spin $\langle E / N\rangle$ e o parâmetro de ordem $O_{h v}$ para as diversas temperaturas para 
$L=56$. Para as temperaturas mais baixas $(T \lesssim 0,780)$, as séries transitam entre duas regiões durante sua evolução, o que é um indício de que o sistema tunela de uma fase para outra. Esse comportamento também pode ser visto nos histogramas das Figuras 5.4(c) e 5.4(d). Para as

\begin{tabular}{ll}
\hline \hline$L$ & \multicolumn{1}{c}{$T_{0}$} \\
\hline 16 & 0,$791 ; 0,830 ; 0,850 ; 0,870$ \\
32 & 0,$780 ; 0,791 ; 0,812 ; 0,825 ; 0,850$ \\
48 & 0,$770 ; 0,780 ; 0,791 ; 0,812 ; 0,870$ \\
56 & 0,$760 ; 0,770 ; 0,780 ; 0,790 ; 0,808 ; 0,860$ \\
72 & 0,$760 ; 0,770 ; 0,780 ; 0,790 ; 0,807 ; 0,830$ \\
\hline \hline
\end{tabular}

Tabela 5.1: Temperaturas $T_{0}$ para produções das séries temporais a partir do algoritmo de Metropolis e utilizadas para repesagem.
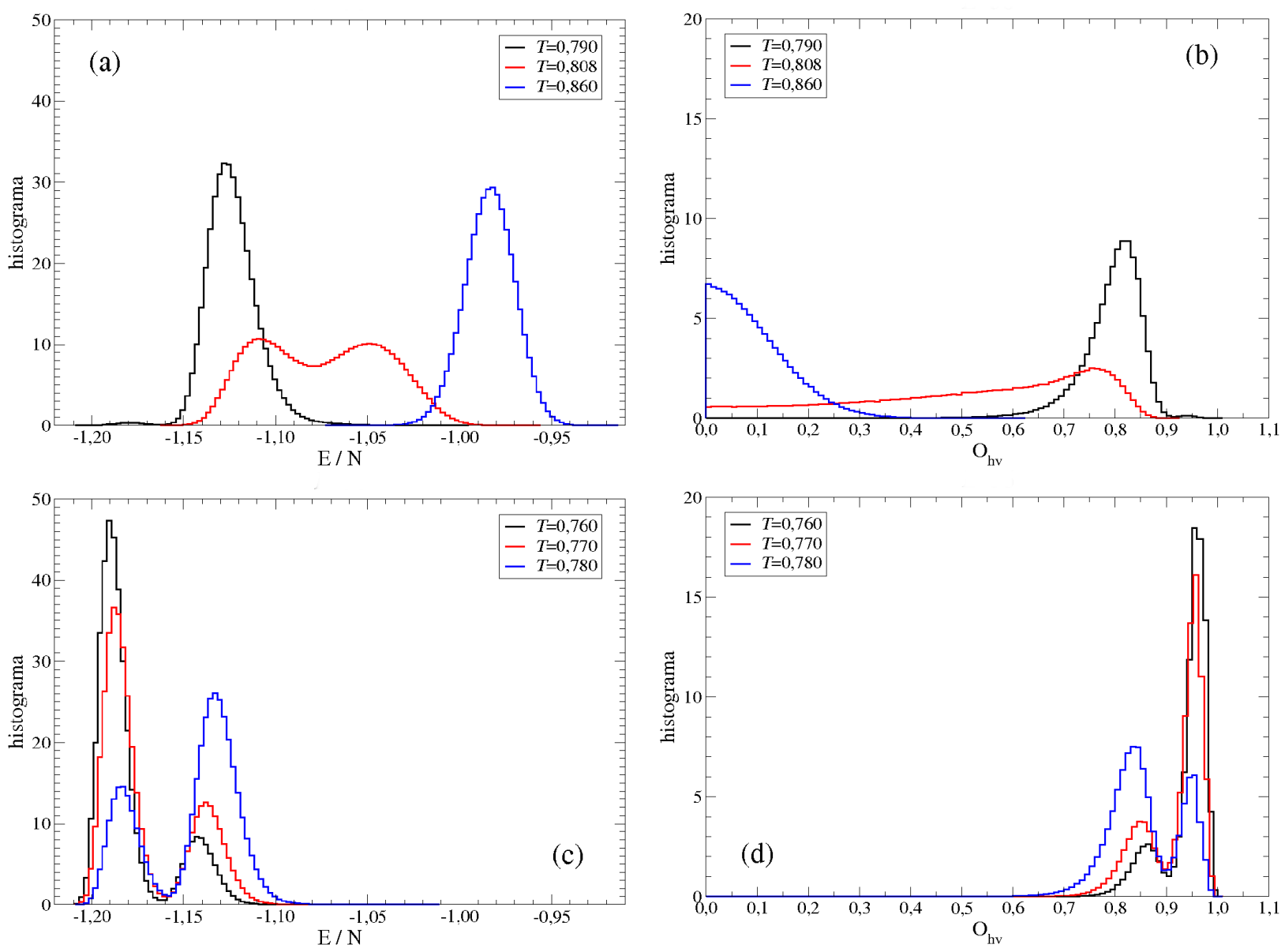

Figura 5.4: Histogramas das séries produzidas para a energia por spin $E / N$ e parâmetro de ordem $O_{h v}$ para $L=56$ em diferentes temperaturas. (a) e (b) correspondem a transição de fase nemáticatetragonal e (c) e (d) à transição de fase faixas-nemática. 
temperaturas mais baixas, observamos claramente a presença de dois picos nos histogramas. Conforme diminuimos a temperatura, observamos que esta estrutura se mantem, ocorrendo apenas alteração nas alturas relativas. O mesmo ocorre para o parâmetro de ordem, o que indica a presença de uma transição de fase. Para temperaturas mais altas, outra transição de fase é sugerida. Em particular, para $T=0,808$ observamos a forma de pico duplo no histograma da Figura 5.4(a). Nesse caso, a altura sendo aproximadamente igual para ambos os picos indica que a temperatura crítica da transição está próxima de 0,808 .

Para identificar as temperaturas críticas de tamanho finito, analisamos o calor específico $C_{v}$ definido em um intervalo (contínuo) de temperaturas por repesagem das séries obtidas a partir das simulações nas temperaturas $T_{0}$. Os resultados para os máximos do calor específico $\left.C_{v}\right|_{\max }$ e as correspondentes temperaturas críticas $T_{c}^{(i)}$ de tamanho finito são apresentados na Tabela 5.2. As temperaturas críticas de tamanho finito $T_{c}^{(i)}$ são definidas pelo máximo do calor específico $C_{v}(L)$. Todas as estimativas das barras de erro foram feitas utilizando a técnica de Jackknife, sendo utilizadas $N_{A}=40$ amostras para $L \leq 56$ e $N_{A}=20$ amostras para $L=72$.

A Figura 5.5 mostra as nossas estimativas finais para as médias da energia por spin $\langle E / N\rangle$ e do calor específico $C_{v}$ em função da temperatura $T$. A Figura 5.5(a) mostra $C_{v}$ para $L=16$, 32 e 48. As estimativas para as barras de erro foram incluídas apenas para $L=48$, no gráfico de dentro da Fig.5.5(a) para ter uma clara apresentação do comportamento de $C_{v}$ para diferentes tamanhos de rede.

Segundo os resultados mostrados na Figura 5.5 para $L=16$, observamos uma transição de fase caracterizada por um pico no calor específico em $T_{c}^{(1)}=0,8323$. Essa transição também é notada pelo comportamento do parâmetro de ordem $O_{h v}$. A partir da análise das configurações obtidas das séries em temperaturas próximas a temperatura de transição, identificamos essa transição como correspondendo a transição entre as fases do tipo faixas de spins e a

\begin{tabular}{lllll}
\hline \hline$L$ & $\left.C_{v}^{(1)}\right|_{\max }$ & $T_{c}^{(1)}$ & $\left.C_{v}^{(2)}\right|_{\max }$ & $T_{c}^{(2)}$ \\
\hline 16 & $3,095(2)$ & $0,8323(2)$ & & \\
32 & $3,92(2)$ & $0,7905(3)$ & & \\
48 & $3,76(3)$ & $0,7785(7)$ & $4,21(1)$ & $0,8132(2)$ \\
56 & $3,72(4)$ & $0,7727(6)$ & $5,44(2)$ & $0,8084(2)$ \\
72 & $4,1(4)$ & $0,767(2)$ & $5,6(1)$ & $0,800(1)$ \\
\hline \hline
\end{tabular}

Tabela 5.2: $T_{c}^{(i)}$ : temperaturas críticas de tamanho finito para as transições de fase observadas para tamanhos de rede $L$ definidas pelo máximo do calor específico $C_{v}^{(i)}{ }_{\max }$ como mostrado nas Fig. 5.5(a) e $5.5(\mathrm{~b})$. 

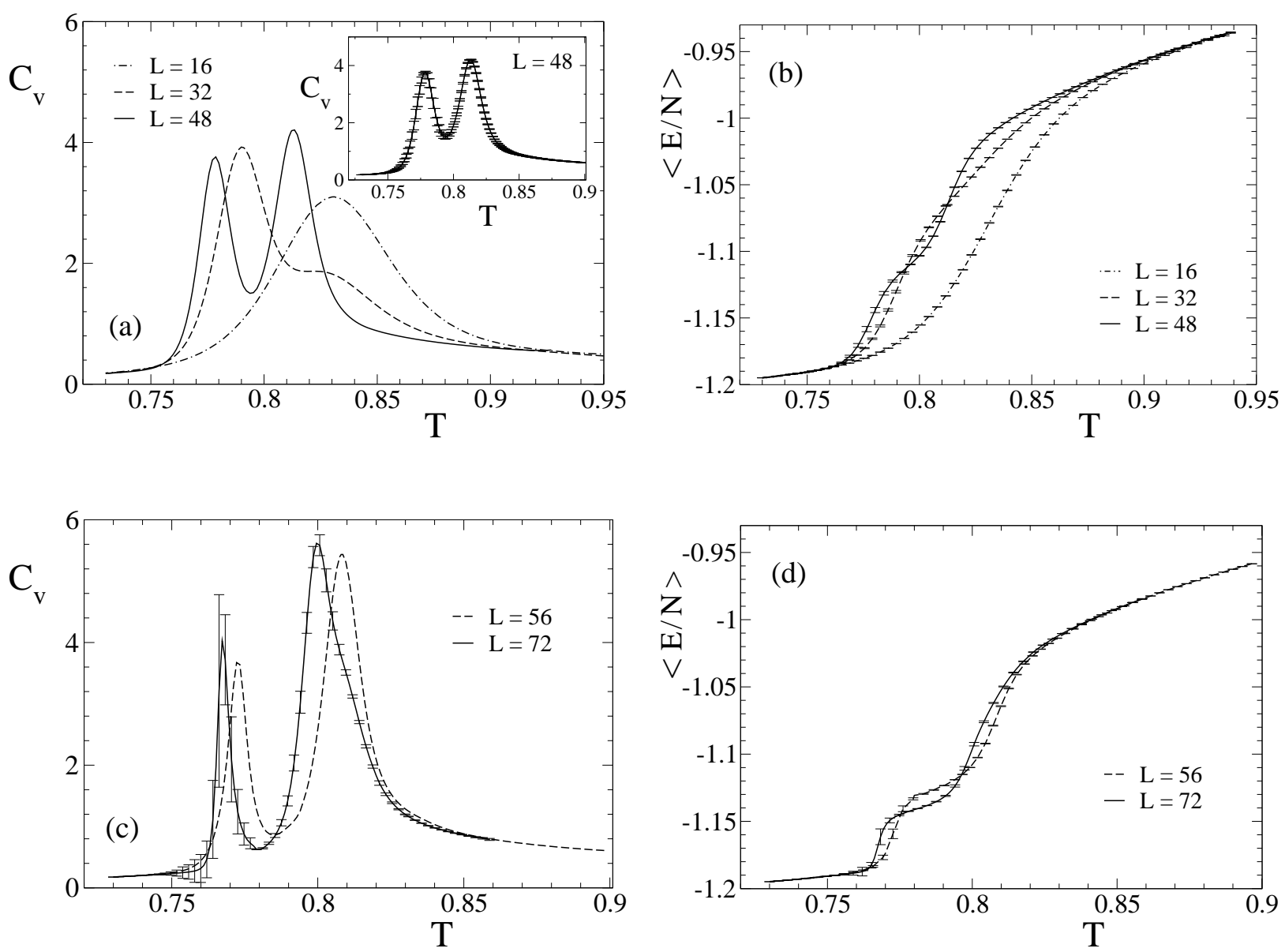

Figura 5.5: Calor específico $C_{v}$ e energia média por spin $\langle E / N\rangle$ em função da temperatura $T$ para $L=16,32,48$ nas figuras (a) e (b), e $L=56,72 \mathrm{em}$ (c) e (d). Dentro da Fig. (a): $C_{v}$ com estimativas de barras de erro para $L=48$.

fase de líquido tetragonal. Conforme aumentamos o tamanho da rede o comportamento das curvas muda drasticamente e uma outra transição aparece. Essa nova transição, a transição entre as fases nemática e tetragonal, apresenta aumento mais pronunciado no máximo do calor específico em $T_{c}^{(2)}$ em comparação com o antigo máximo agora em $T_{c}^{(1)} \approx 0,77$. Esta nova temperatura $T_{c}^{(1)}$ corresponde à transição faixas-nemática. A Figura 5.6 ilustra as configurações típicas em cada uma das fases para $L=56$. Esta figura mostra como as simetrias direcional e translacional são perdidas com o aumento da temperatura em direção à fase tetragonal. As configurações típicas nas temperaturas de transição são exibidas na Figura 5.7.

Notamos que a posição dos picos do calor específico muda conforme $L$ aumenta. Esta mudança ocorre igualmente para ambos os picos na direção de temperaturas mais baixas. Esse é um efeito causado pelo tamanho finito da rede. Considerando que esperamos observar as duas transições de fase no limite termodinâmico e levando em conta os resultados numéricos 
obtidos na Tabela 5.2 para as temperaturas críticas de tamanho finito, supomos que a diferença entre as temperaturas críticas atingirá um valor fixo.

Apresentamos o máximo das susceptibilidades e respectivas temperaturas críticas de tamanho finito $T_{c}^{(i)}$ na Tabela 5.3. Estas temperaturas são definidas pela ocorrência de máximos

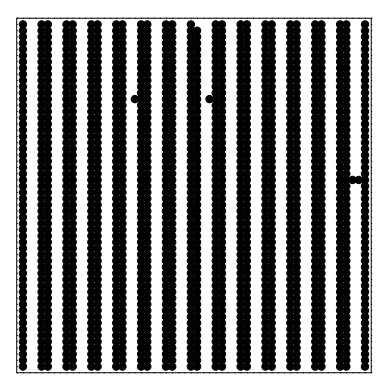

(a) $T=0,710$

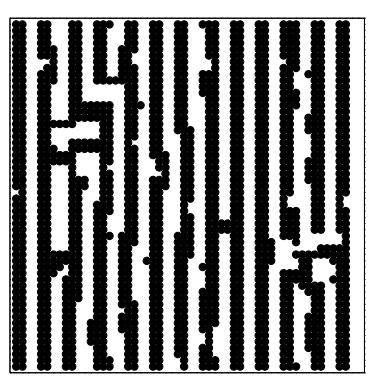

(b) $T=0,790$

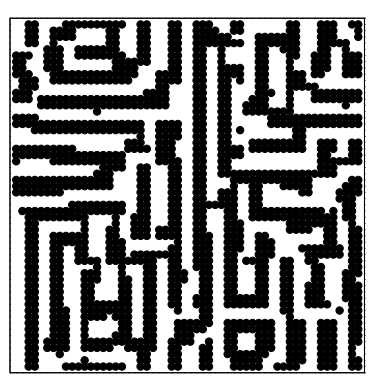

(c) $T=0,840$

Figura 5.6: Configurações típicas de spins para $L=56$ em temperaturas nas fases: (a) faixas, (b) nemática e (c) tetragonal. A configuração na fase do tipo faixas representa $E / N=-1,2028$, $O_{h v}=0,9873$; na fase nemática, $E / N=-1,1172, O_{h v}=0,7903$; e na fase líquido tetragonal $E / N=-1,0201$ e $O_{h v}=0,1777$.

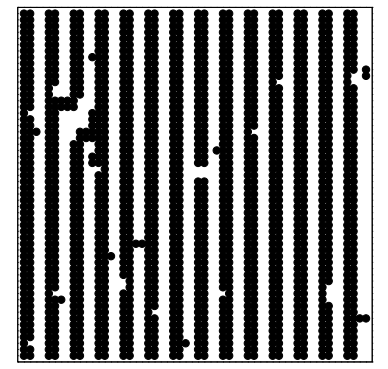

(a) $T=0,773$

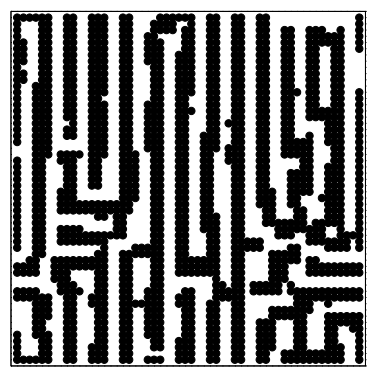

(b) $T=0,808$

Figura 5.7: Configurações de spins nas temperaturas de transição para $L=56$. A configuração apresentada em (a) tem $E / N=-1,1670, O_{h v}=0,9205$; enquanto que em (b), $E / N=-1,0769$ e $O_{h v}=0,5633$.

\begin{tabular}{cllll}
\hline \hline$L$ & $\chi\left(O_{h v}\right)_{\max }^{(1)}$ & \multicolumn{1}{c}{$T_{c}^{(1)}$} & $\chi\left(O_{h v}\right)_{\max }^{(2)}$ & $T_{c}^{(2)}$ \\
\hline 16 & $28,42(2)$ & $0,8336(2)$ & & \\
32 & $49,4(1)$ & $0,8258(2)$ & & \\
48 & $17,0(5)$ & $0,781(2)$ & $129,7(6)$ & $0,8140(2)$ \\
56 & $13,8(2)$ & $0,773(1)$ & $170(2)$ & $0,8097(2)$ \\
72 & $13,1(9)$ & $0,768(2)$ & $209(3)$ & $0,809(2)$ \\
\hline \hline
\end{tabular}

Tabela 5.3: Máximos da susceptibilidade e as correspondentes temperaturas críticas $T_{c}^{(i)}$ identificadas na Fig. 5.8. 
na susceptibilidade $\chi(T)$ como mostrado na Fig. 5.8(a) e (c). As Figuras 5.8(b) e (d) ilustram o comportamento do parâmetro de ordem $O_{h v}$ como função da temperatura para diferentes tamanhos de rede. Elas mostram que a quebra da simetria direcional acontece principalmente na segunda temperatura de transição termodinâmica em $T_{c}^{(2)}=0,809$. Os picos nas susceptibilidades relacionados a esta segunda transição tornam-se maiores a medida que o tamanho da rede aumenta. Por outro lado, observamos valores menores para os máximos associados à primeira transição termodinâmica $T_{c}^{(1)}$ à medida que o tamanho da rede aumenta. Estes máximos ocorrem em $T_{c}^{(1)} \approx 0,77$. Segundo as estimativas da Tabela 5.3, podemos observar uma tendência decrescente não usual dos picos. Essa tendência pode continuar até alcançar um valor limite ou ainda, pode continuar até resultar em que essa transição seja apenas um artefato da rede. Em qualquer caso, essa nova propriedade coloca um grau extra de dificuldade na identificação da natureza da transição. Essa situação apresenta um comportamento paralelo quando analisamos os máximos do calor específico na Tabela 5.2. A primeira transição
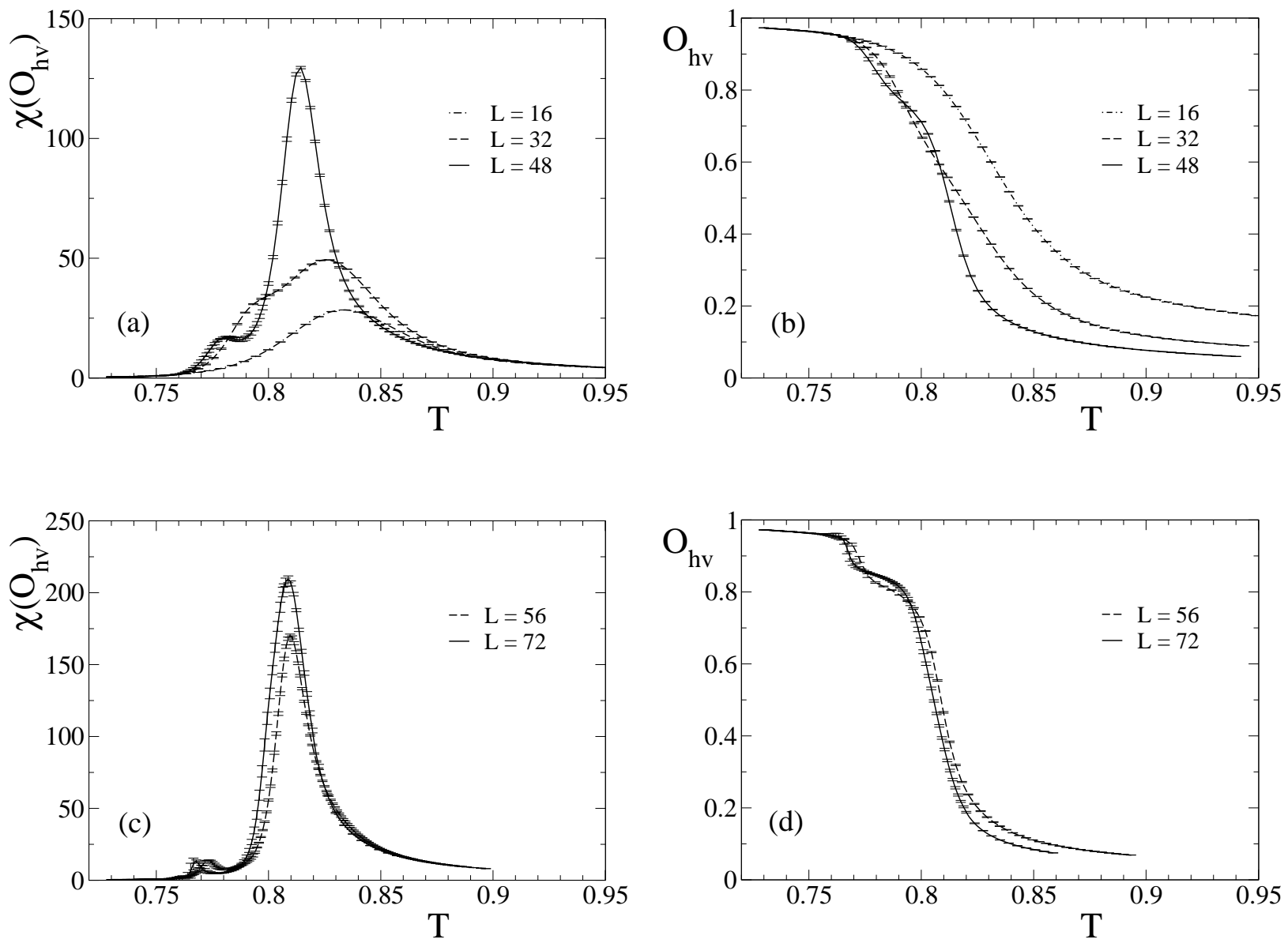

Figura 5.8: Susceptibilidade e parâmetro de ordem para diversos tamanhos de rede em função da temperatura. 
$\left(T_{c}^{(1)} \approx 0,77\right)$ parece apresentar picos no calor específico tendo a mesma altura, enquanto que a segunda transição $\left(T_{c}^{(2)} \approx 0,809\right)$ mostra picos cada vez maiores conforme o tamanho da rede aumenta.

Desde que a fenomenologia desse modelo revela-se somente para grandes tamanhos de rede, nossos dados ainda apresentam limitações para uma análise adequada de escala de tamanho finito. Qualquer simulação intensiva de Monte Carlo com algoritmos de atualização local sofrerá severas limitações. No entanto, nossos dados permitem apresentar algumas estimativas. Para este fim, consideramos apenas os três últimos valores para o máximo de $C_{v}$ na Tabela 5.2 e para o máximo de $\chi\left(O_{h v}\right)$ na Tabela 5.3, correspondendo a $L=48,56$ e 72 . Uma vez que os picos no calor específico e na susceptibilidade relacionados com a primeira transição parecem convergir para um valor constante, assumimos $\alpha \approx 0$ e $\gamma \approx 0$. Neste caso, correções logarítmicas devem ser levadas em conta para descrever os máximos. A análise de escala de tamanho finito para a segunda transição fornece $\alpha=0,87(1), \gamma=0,69(6)$ e $\nu=0,57(7)$. Esses resultados suportam o caráter de segunda ordem para a transição de fase nemáticatetragonal. A natureza contínua da transição nemática-tetragonal estaria de acordo com o sugerido teoricamente por Abanov e coautores [30], através de um modelo contínuo, e por Pighin e Cannas [21], a partir de uma abordagem de campo médio. Porém, Cannas e coautores [33] apresentaram fortes indícios de que essa transição é de primeira ordem para $\delta=2$. Nesse mesmo trabalho, Cannas e coautores especulam sobre a hipótese de uma transição do tipo Kosterlitz-Thouless (KT) como uma alternativa à possibilidade do caráter de primeira ordem encontrado. Notamos que o nosso resultado para o calor específico $C_{v}$ não apresenta um comportamento divergente, ou mesmo efeitos de tamanho finito apreciáveis, então a hipótese de uma transição do tipo KT também precisa ser considerada. No entanto, nossos resultados apresentam grandes barras de erro para o calor específico no caso $L=72$, dificultando conclusões mais precisas. Essa falta de precisão no calor específico, quando comparamos com os resultados para o segundo pico em $T_{c}^{(2)}$, está relacionada à forte autocorrelação na transição faixas-nemática, como apresentam nossos resultados a seguir sobre o tempo de autocorrelação integrado $\tau_{\text {int }}$ nas simulações de Metropolis.

Nossos cálculos de $2 \tau_{\text {int }}$ são mostrados na Figura 5.9 para redes de tamanhos $L=16,32,48$ e 56, onde introduzimos a notação $k$ para descrever amostras de tamanho $n_{\tau}$, sendo $n_{\tau}=2^{k}$. Obtemos $2 \tau_{\text {int }} \approx 800$ medições $(L=16)$ para as temperaturas próximas a temperatura crítica de tamanho finito e valores menores para temperaturas correspondendo à fase tetragonal. Para o caso onde $L=32$, a Figura 5.9(b) mostra um valor maior: $2 \tau_{\text {int }}=6 \times 10^{4}$ (gráfico de dentro da Figura 5.9(b)) para $T=0,780$ na fase de faixas e $4 \times 10^{4}$ para $T=0,791$, que corresponde 

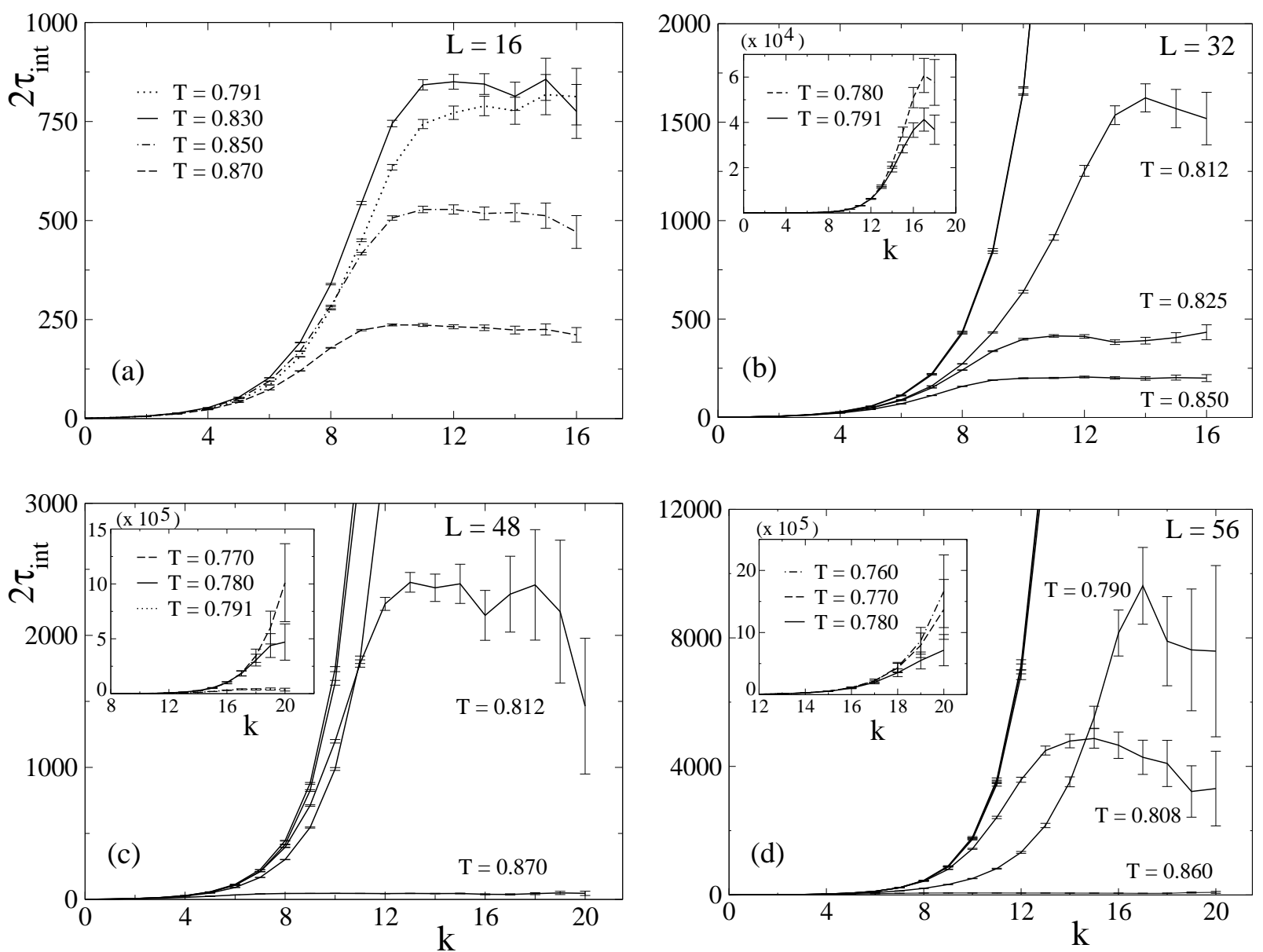

Figura 5.9: Tempo de autocorrelação integrado $2 \tau_{\text {int }}$ para tamanhos de rede $L=16$ (a), $L=32$ (b), $L=48$ (c) e $L=56$ (d) em função do tamanho das séries temporais $2^{k}$.

ao máximo do calor específico.

Na Figura 5.9(c) apresentamos nossos resultados para $L=48$, cujos dados para o calor específico apresentam duas temperaturas críticas. Para a temperatura quando o sistema se encontra na fase de faixas $(T=0,770)$, obtemos um tempo de autocorrelação maior, quando comparado à transição faixas-nemática: $2 \tau_{\text {int }}(T=0,780)=5 \times 10^{5}$, enquanto que na fase nemática, obtemos $2 \tau_{\text {int }}(T=0,791)=4 \times 10^{4}$. Aumentando a temperatura para a próxima transição, obtemos $2 \tau_{\text {int }}(T=0,812)=2,4 \times 10^{3}$. Para uma temperatura ainda mais alta, observamos uma forte diminuição da $2 \tau_{\text {int }}$, atingindo valores tão pequenos quanto 45 para $2 \tau_{\text {int }}$. A Figura 5.9(d) mostra um valor grande para este tempo de autocorrelação, superior a $2 \times 10^{6}$ para série de dados coletados na fase de faixas e na transição faixas-nemática. Na transição de fase nemática-tetragonal obtemos $2 \tau_{\text {int }}(T=0,808)=5 \times 10^{3}$ e um menor valor 60 na temperatura $T=0,860$, a qual o sistema encontra-se na fase tetragonal.

Podemos descrever o tempo de autocorrelação nas séries do observável energia obtidas 
nas temperaturas de transição como uma simples lei de potência $2 \tau_{\text {int }, E}=A L^{z_{\text {int }}}$, onde $z_{\text {int }}$ é o expoente crítico dinâmico associado [41]. Uma estimativa grosseira fornece $z_{\text {int }} \approx 6,2 \mathrm{e}$ $z_{i n t} \approx 4,6$, respectivamente, para as transições faixas-nemática e nemática-tetragonal. Esses são valores elevados, uma vez que algoritmos locais de Monte Carlo geralmente têm o expoente crítico dinâmico $z \approx 2$ [54]. Para os algoritmos de atualização não-locais como o algoritmo de Swendsen-Wang, este expoente é ainda menor, apresentando para o modelo de Ising bidimensional puro $z=0,222(7)$ [54].

A partir da análise do tempo de autocorrelação podemos confirmar a existência de estados com longos períodos de estabilidade próximos e na transição faixas-nemática. Recentemente, a existência desses estados foi destacada em um estudo realizado por Cannas e coautores [55]. Eles encontraram fortes meta-instabilidades associadas a transição faixas-nemática. A permanência do sistema nesses estados leva a barras de erro grandes para as funções resposta nessa transição quando comparada com a transição nemática-tetragonal, para a qual os valores de $\tau_{\text {int }}$ são ordens de magnitude menores.

\subsubsection{Algoritmo multicanônico}

Para todos os resultados apresentados nessa seção nós utilizamos a energia mínima por partícula $e_{\min }=-1,22$ e o número de energias considerado $M=L^{2}$. Esta valor de $e_{\min }$ foi escolhido com base no valor da energia das configurações para o estado fundamental do tipo faixas encontradas na séries obtidas através do algoritmo de Metropolis (vide Figura 5.2(b)). Nós definimos as energias $E_{\eta}$ de acordo com a relação (4.1.6) descrita na seção 4.1, ou seja,

$$
E_{\eta}=e_{\min } \times L^{2}+\eta \epsilon .
$$

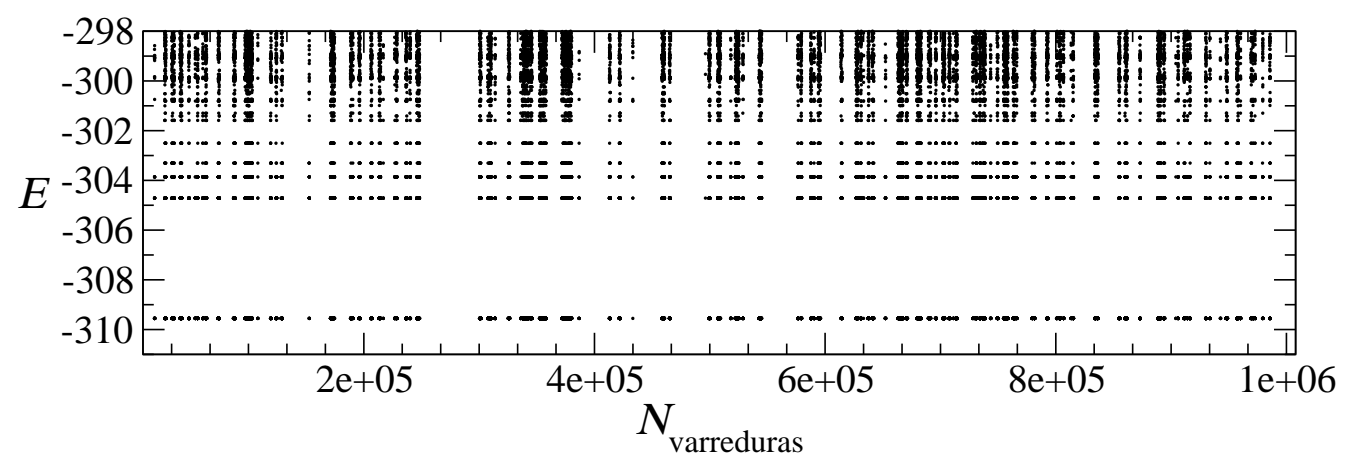

Figura 5.10: Série de Monte Carlo obtida por meio do algoritmo multicanônico para $L=16$. 
Para a determinação dos parâmetros multicanônicos $a(E)$ e $b(E)$, para $L=16$, efetuamos 300 atualizações a partir dos histogramas resultantes de simulações com $5 \times 10^{4}$ varreduras cada uma, segundo a metodologia descrita na seção 4.3. Primeiro obtivemos os valores para os parâmetros multicanônicos utilizando $\epsilon=2$, porém, os valores de $a(E)$ e $b(E)$ apresentaram muitas oscilações. A causa das oscilações é a existência de uma vacância entre as energias -305 e -309, como mostra a Figura 5.10. Uma vacância como essa, maior que o valor de $\epsilon$, implica que nenhuma configuração nesse intervalo de energia será amostrada, assim o histograma da energia correspondente será igual ao valor mínimo $h_{0}$ (vide expressão (4.3.15) da seção 4.3). Como os parâmetros multicanônicos para uma dada energia são determinados utilizando

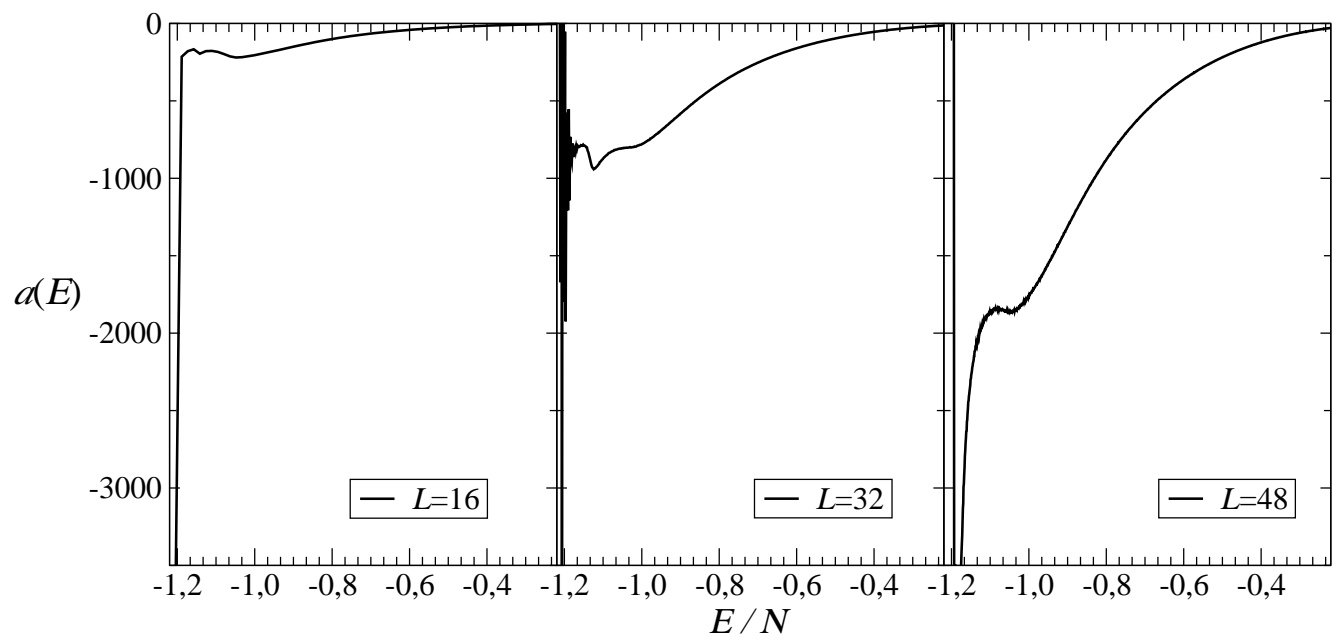

Figura 5.11: Parâmetros multicanônicos $a(E)$ em função da energia por spin $E / N$ para diferentes tamanhos de rede $L$.

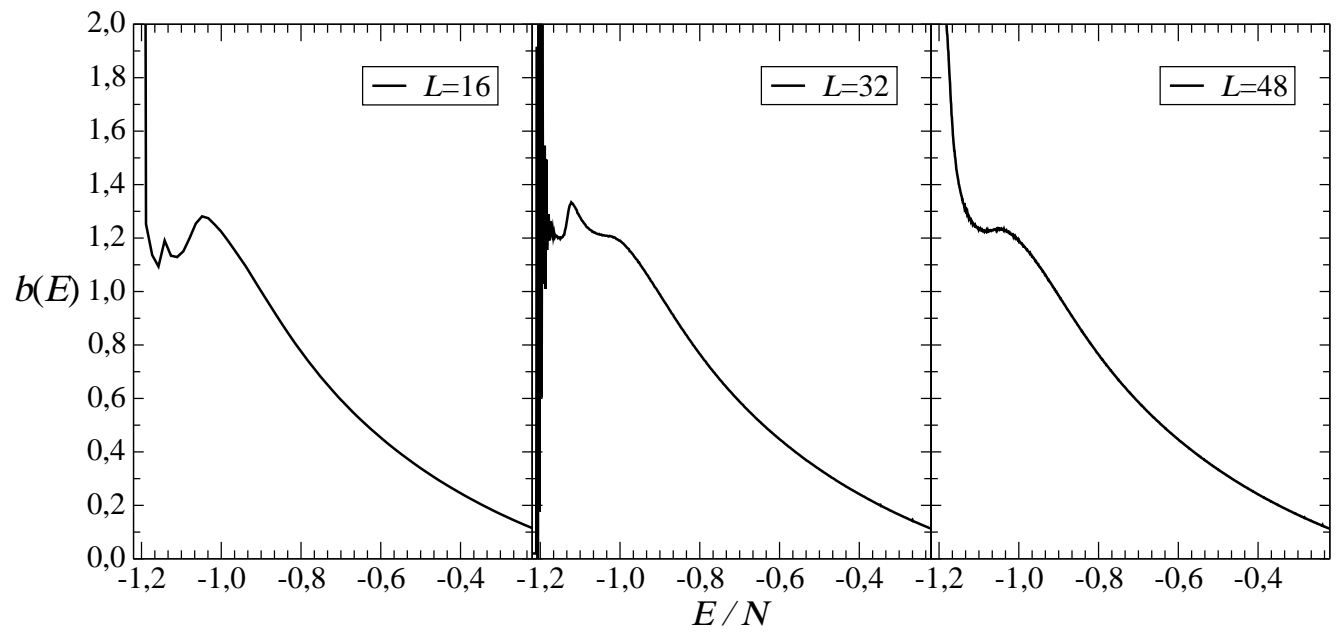

Figura 5.12: Parâmetros multicanônicos $b(E)$ em função da energia por spin $E / N$ para diferentes tamanhos de rede $L$. 
histogramas das energias vizinhas, as oscilações aparecem. Afim de evitar essas oscilações nos valores de $a(E)$ e $b(E)$ para as energias mais próximas do estado fundamental, escolhemos $\epsilon=4$. Como podemos notar pelas Figuras 5.11 e $5.12(L=16)$, respectivamente, os valores dos parâmetros $a(E)$ e $b(E)$ não oscilam para este valor de $\epsilon$. Utilizando esses parâmetros produzimos uma série com $6 \times 10^{6}$ varreduras. Apresentamos na Figura 5.13(a) o histograma da série produzida para a energia. Notamos que, exceto para energias próximas à energia do estado fundamental, o histograma apresenta um valor aproximadamente constante, o que mostra que configurações com energias distintas foram visitadas um mesmo número de vezes. Isso indica que a produção da série utilizando os pesos $a(E)$ e $b(E)$ é satisfatória de acordo com o esperado para o algoritmo multicanônico.

A determinação dos parâmetros multicanônicos para $L=32$ foi feita realizando 400 atualizações a partir dos histogramas resultantes de simulações tendo $5 \times 10^{4}$ varreduras cada uma.

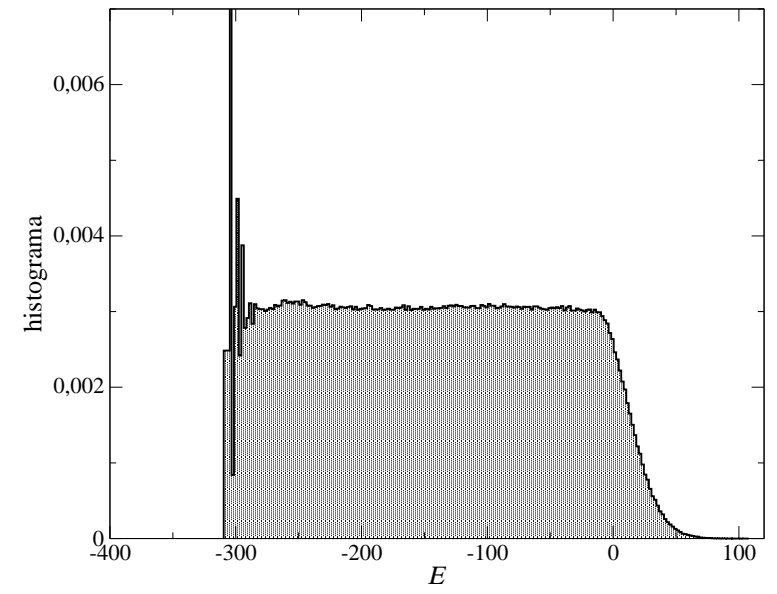

(a) $L=16$

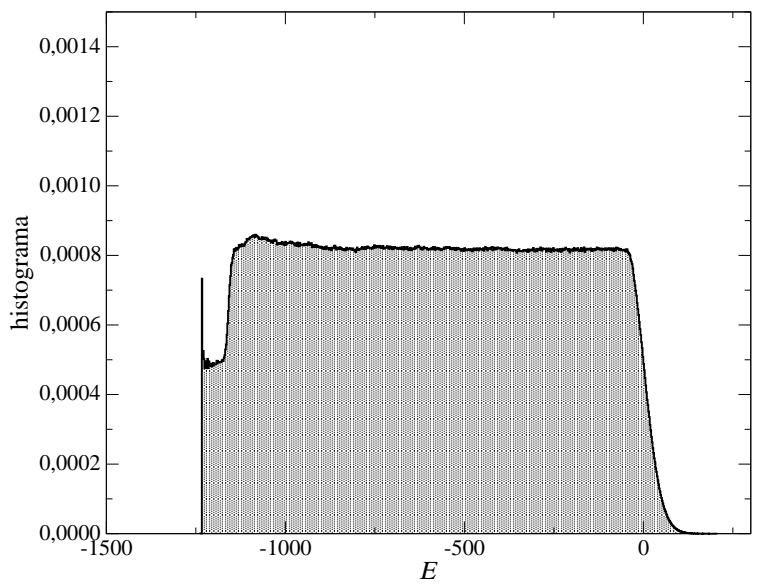

(b) $L=32$

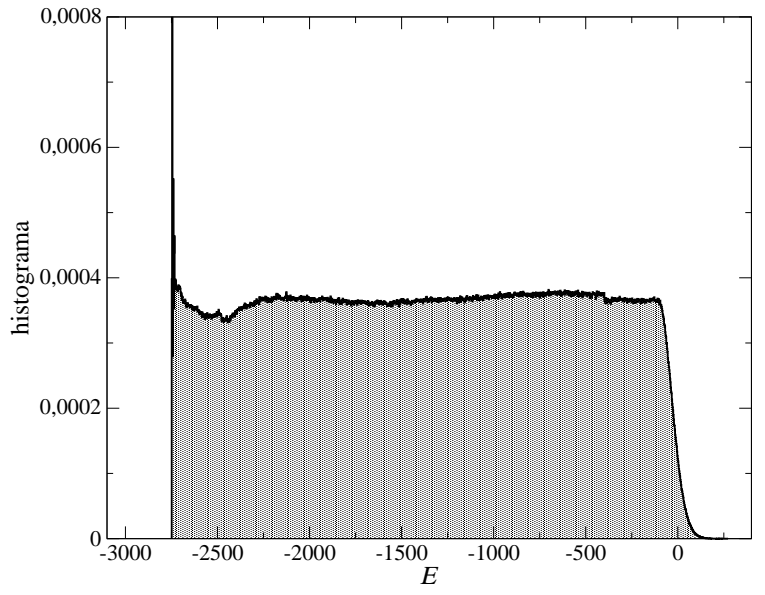

(c) $L=48$

Figura 5.13: Histogramas para as séries produzidas utilizando o algoritmo multicanônico para diferentes tamanhos de rede. 
Nesse caso, utilizamos $\epsilon=2$. Este valor de $\epsilon$ mostrou-se adequado pois diferença entre as energias do estado fundamental e do primeiro estado excitado é menor que 2. Contudo, também observamos um comportamento "ruidoso" para os valores dos parâmetros $a(E)$ e $b(E)$, como mostram as Figuras 5.11 e 5.12, respectivamente. Utilizando esses valores para os parâmetros multicanônico, produzimos séries temporais para a energia e parâmetro de ordem com $6 \times 10^{7}$ varreduras. Na Figura 5.13(b) mostramos o histograma para a série temporal produzida. O histograma apresenta um comportamento aproximadamente plano para a maioria das energias, porém, observamos uma diminuição na amostragem das configurações com energias mais baixas, próximas à energia do estado fundamental.

Para $L=48$, a determinação dos parâmetros multicanônicos foi feita realizando 460 atualizações a partir dos histogramas resultantes de simulações tendo $5 \times 10^{4}$ varreduras cada uma. Nesse caso, também utilizamos $\epsilon=2$. Um teste foi realizado utilizando $\epsilon=4$ e percebemos que para $\epsilon=2$ a atualização "caminha" mais rapidamente em direção às energias mais baixas. Além disso, fazendo um gráfico dos valores de $a(E)$ e $b(E)$ em função da energia por spin $E / N$ para os dois casos, nenhuma diferença significativa foi notada. A Figura 5.14 mostra os histogramas para as séries em diferentes estágios da atualização dos parâmetros multicanônicos utilizando $\epsilon=2$. Os valores dos parâmetros $a(E)$ e $b(E)$ após as 460 atualizações são mostrados nas Figuras 5.11 e 5.12, respectivamente. Vale dizer que os valores dos parâmetros para 400 atualizações não diferem em nada em relação aos valores dos parâmetros utilizados

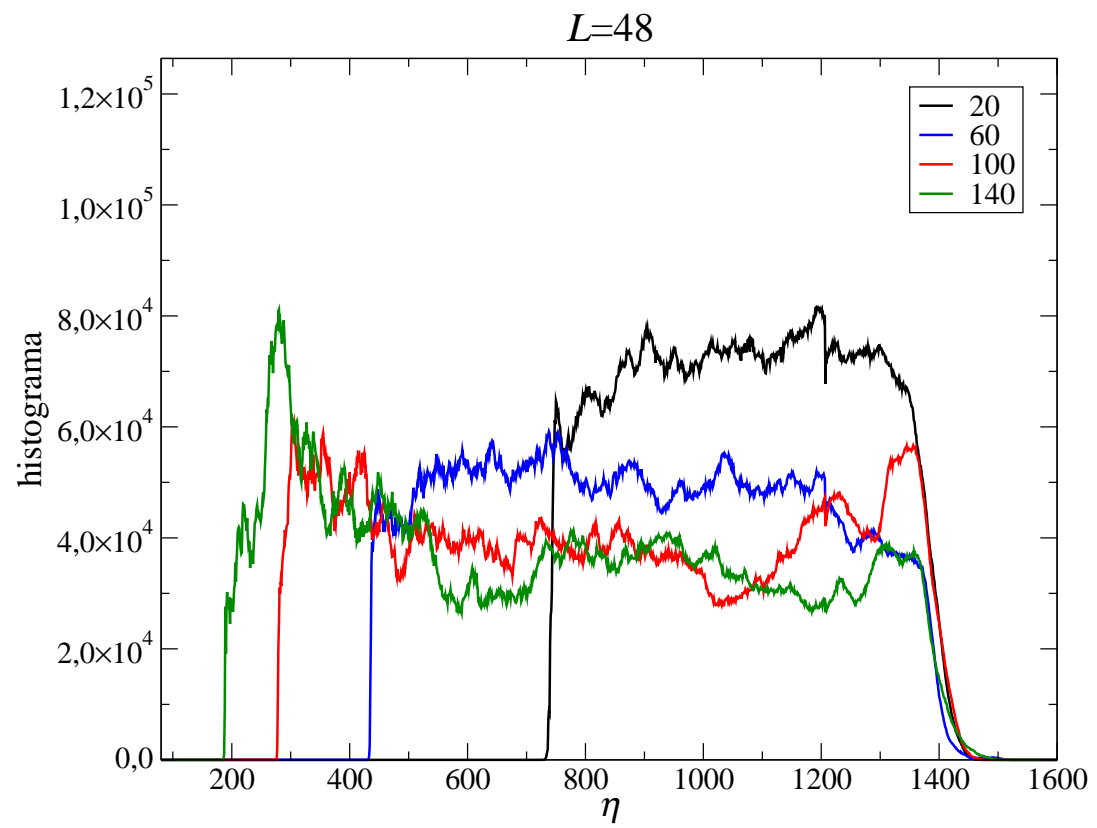

Figura 5.14: Histogramas para as séries produzidas em diferentes estágios da atualização $(20$ - 140 atualizações) dos parâmetros $a(E)$ e $b(E)$ para a rede de tamanho $L=48$. 
para a produção da série temporal final (460 atualizações). Utilizando esses parâmetros produzimos uma série temporal com $4 \times 10^{7}$ varreduras. O histograma para essa série é mostrado na Figura 5.13(c). Novamente, notamos um comportamento uniforme no histograma, como esperado para o histograma obtido a partir de uma série temporal produzida pelo algoritmo multicanônico.

Utilizando a técnica de repesagem nas séries produzidas para os tamanhos de rede $L=16$, 32 e 48, obtivemos as grandezas físicas em função da temperatura. Os gráficos dessas grandezas são apresentados na Figura 5.15

Para $L=16$, percebemos uma diferença entre as energias da fase do tipo faixas calculadas a partir das repesagem (em $T=0,75)$ das séries obtidas por meio do algoritmo multicanônico

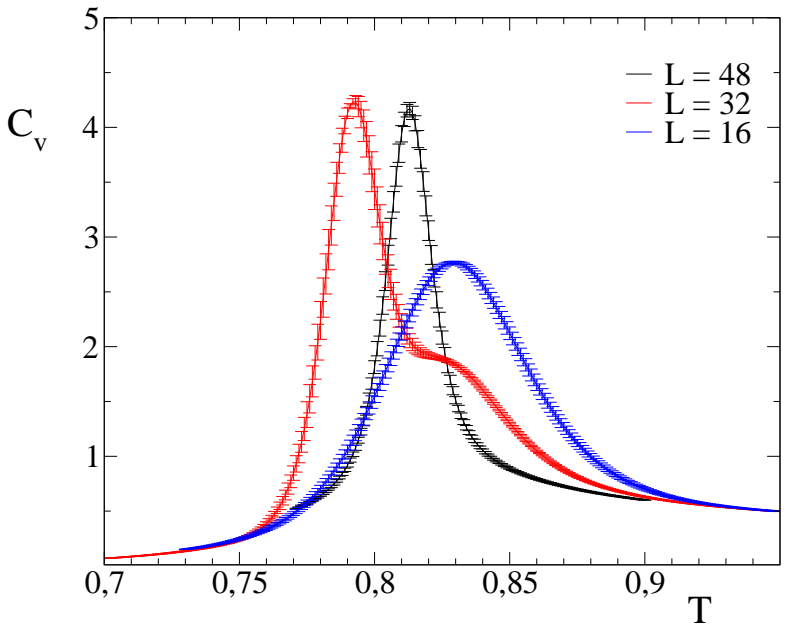

(a) Calor específico $C_{v}$

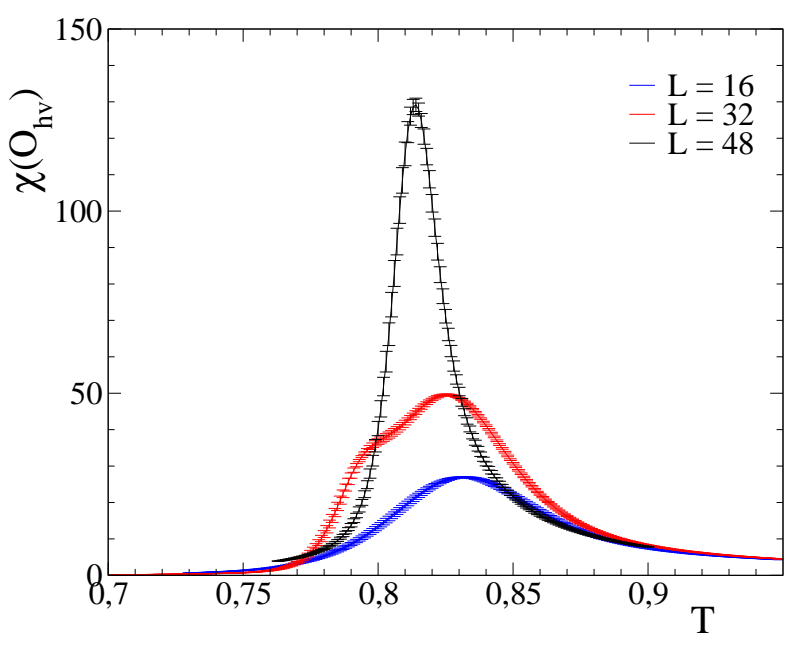

(c) Susceptibilidade $\chi\left(O_{h v}\right)$

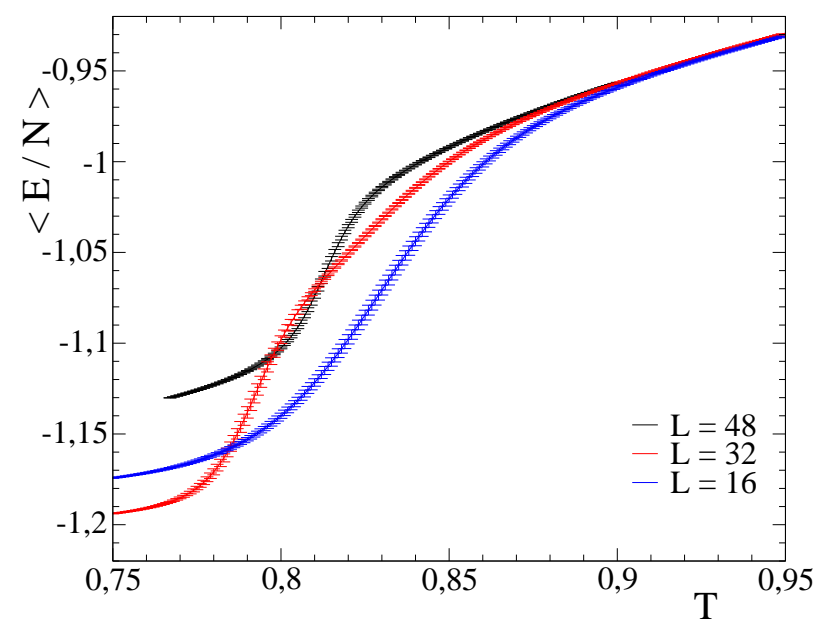

(b) Energia por spin $\langle E / N\rangle$

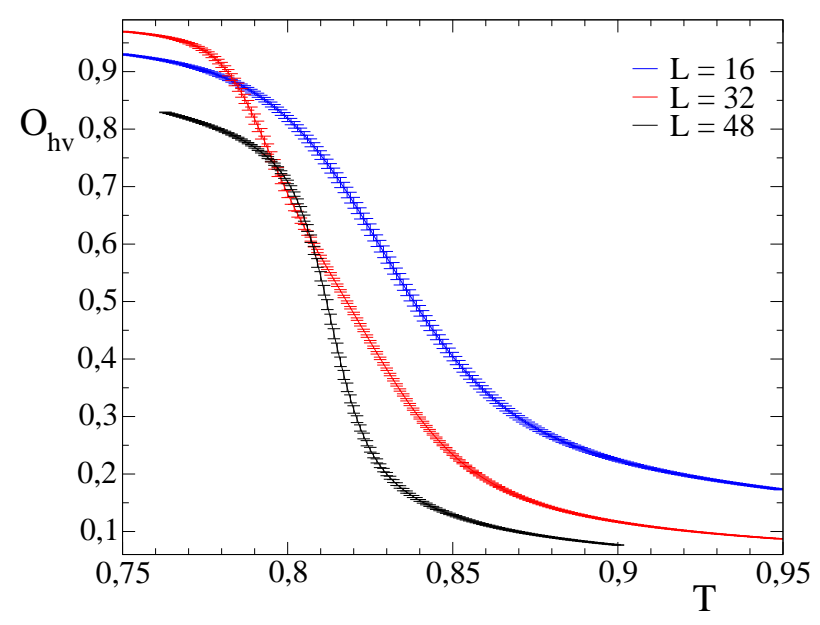

(d) Parâmetro de ordem $O_{h v}$

Figura 5.15: Grandezas termodinâmicas em função da temperatura $T$ resultantes da repesagem das séries obtidas pelo algoritmo multicanônico para redes de tamanho $L=16,32$ e 48 . 
$(\langle E / N\rangle \approx 1,174)$ e por meio do algoritmo de Metropolis $(\langle E / N\rangle \approx 1,190)$. É possível que isso tenha ocorrido por causa da escolha do valor de $\epsilon=4$. Para esse caso, a discretização grosseira das energias altera os valores de $a(E)$ e $b(E)$, os quais são utilizados para a repesagem. Consequentemente, os valores dos parâmetros $a(E)$ e $b(E)$ para as energias das configurações da fase do tipo faixas são levadas em consideração como sendo iguais aos valores dos parâmetros $a(E)$ e $b(E)$ correspondentes à energias mais altas, o que altera as estimativas para as energias repesadas para as temperaturas mais baixas. Isso também influencia as estimativas das outras grandezas observáveis, como mostra a Figura 5.15.

Apresentamos na Tabela 5.4 os resultados para a posição e altura dos picos do calor específico para $L=16,32$ e 48. Na Tabela 5.5 apresentamos os resultados para a posição e altura dos picos da susceptibilidade do parâmetro de ordem. Comparando os dois algorítmos, percebemos que os melhores resultados quantitativos são em relação aos máximos da susceptibilidade e as temperaturas de transição obtidas por meio dessa grandeza.

Comparando os resultados dos dois algoritmos para os diferentes tamanhos de rede observamos que os resultados mais próximos são para $L=32$. Apesar dos máximos para o calor específico não estarem exatamente na mesma temperatura crítica nem possuirem exatamente a mesma altura, as curvas obtidas para as grandezas físicas são bastante parecidas. Para $L=48$,

\begin{tabular}{ccccc}
\hline \hline$L$ & $\left.C_{v}^{(1)}\right|_{\max }$ & $T_{c}^{(1)}$ & $\left.C_{v}^{(2)}\right|_{\max }$ & $T_{c}^{(2)}$ \\
\hline 16 & $2,77(1)$ & $0,8291(5)$ & & \\
32 & $4,23(3)$ & $0,7923(5)$ & & \\
48 & & & $4,17(5)$ & $0,8130(5)$ \\
\hline \hline
\end{tabular}

Tabela 5.4: Temperaturas críticas de tamanho finito para as transições de fase observadas para tamanhos de rede $L$ definidas pelo máximo de calor específico $\left.C_{v}\right|_{\max }$ obtidos através do algoritmo multicanônico, como mostrado nas Figura 5.15(a).

\begin{tabular}{llccc}
\hline \hline$L$ & $\chi\left(O_{h v}\right)_{\max }^{(1)}$ & $T_{c}^{(1)}$ & $\chi\left(O_{h v}\right)_{\max }^{(2)}$ & $T_{c}^{(2)}$ \\
\hline 16 & $26,9(1)$ & $0,832(1)$ & & \\
32 & $49,6(2)$ & $0,826(1)$ & & \\
48 & & & $129(2)$ & $0,814(1)$ \\
\hline \hline
\end{tabular}

Tabela 5.5: Temperaturas críticas de tamanho finito para as transições de fase observadas para tamanhos de rede $L$ definidas pelo máximo da susceptibilidade do parâmetro de ordem $\chi\left(O_{h v}\right)_{\max }^{(i)}$ obtidos através do algoritmo multicanônico, como mostrado nas Figura 5.15(c). 


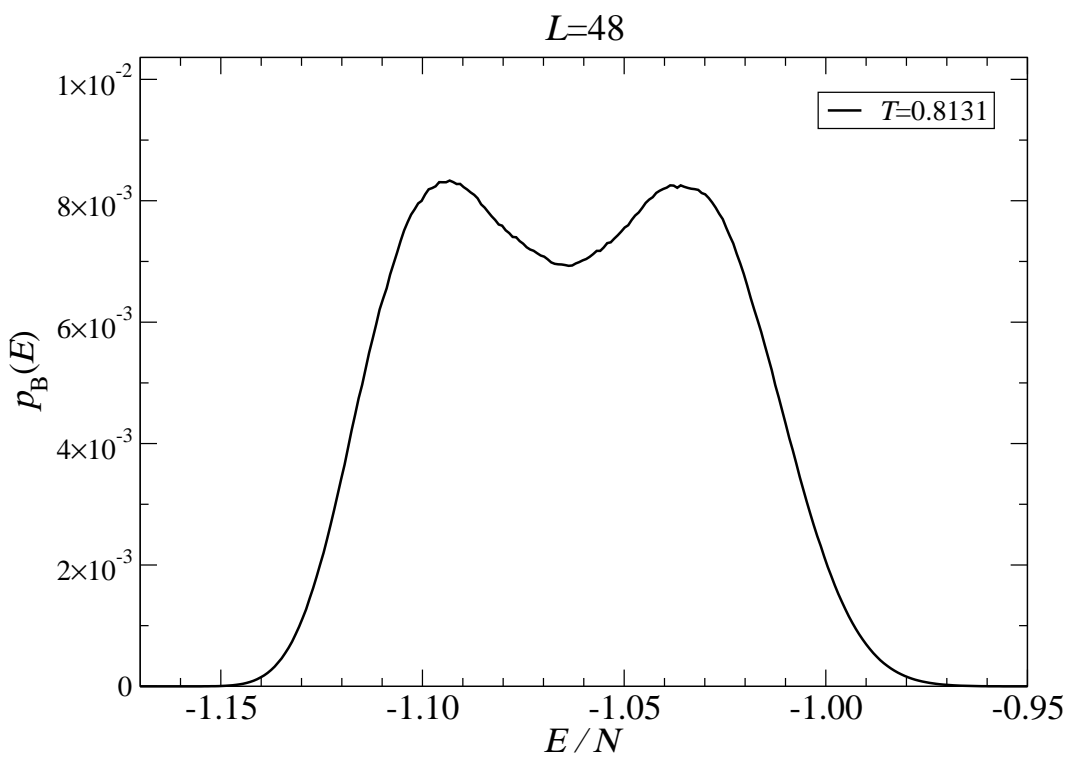

Figura 5.16: Probabilidade canônica de Gibbs na temperatura crítica de tamanho finito $T_{c}^{(2)}=$ 0,8131 , para $L=48$, calculada através da equação (4.3.8) a partir dos parâmetros multicanônicos $a(E)$ e $b(E)$.

porém, uma grande diferença é notada. Os gráficos obtidos não apresentam a transição em $T_{c}^{(1)}$ correspondendo à transição de fase faixas-nemática. Percebemos, contudo, que os valores de $a(E)$ e $b(E)$ para energias bem próximas ao estado fundamental não foram atualizados em relação aos valores iniciais $a^{0}(E)$ e $b^{0}(E)$. Isso ocorreu pois na determinação dos parâmetros as simulações não visitaram esses estados. Talvez uma determinação mais acurada dos parâmetros resolvesse esse problema. Contudo, em específico para $L=48$, nenhuma diferença foi observada para os parâmetros $a(E)$ e $b(E)$ quando comparamos os resultados com 460 atualizações de $5 \times 10^{4}$ varreduras e os obtidos após 505 atualizações de $1 \times 10^{5}$ varreduras. Talvez aumentando ainda mais o número de varreduras para cada atualização resolva o problema, contudo, o tempo computacional gasto nessas simulações é bastante alto (vide Apêndice B), portanto, essa é uma opção para um trabalho futuro.

Por outro lado, poderíamos associar o parâmetro $b(E)$ a uma espécie de "temperatura". Essa relação pode ser verificada empiricamente, pois uma espécie de transição é identificada na curva $b(E)$ da Figura $5.12(L=48)$ em $b(E) \approx 1,23$. O inverso desse valor é aproximadamente 0,8130, que é justamente o valor da temperatura onde observamos um máximo no calor específico. Porém, para o valor de $b(E)=1,2845$, onde a transição faixas-nemática era esperada $^{7}$, não observamos nenhum comportamento desse tipo. Fato que ainda carece de

\footnotetext{
${ }^{7}$ Esse valor de $b(E)$ corresponde ao inverso da temperatura crítica $T=0,7785$ para o máximo do calor específico com $L=48$ obtido por meio do algoritmo de Metropolis.
} 
explicação e somente a partir de trabalhos futuros poderá ser esclarecido.

Como exemplo do uso do algoritmo multicanônico, calculamos a probabilidade canônica de Gibbs na temperatura crítica de tamanho finito $T_{c}^{(2)}=0,8131$ para $L=48$. Fizemos isso utilizando a equação (4.3.8) partindo dos parâmetros multicanônicos $a(E)$ e $b(E)$. Observamos a presença do duplo pico indicando a transição nemática-tetragonal. 


\section{Capítulo 6}

\section{Conclusões}

Neste capítulo apresentamos as principais conclusões do estudo realizado sobre o modelo de Ising dipolar bidimensional.

Para baixas temperaturas, identificamos a fase do tipo faixas de spins em configurações correspondentes à energia do estado fundamental para o caso $\delta=2$. Analisando essas configurações observamos faixas com largura $h=2$ para todos os tamanhos de rede. Como mencionamos na seção 3.2, esse resultado concorda com os resultados de cálculos analíticos [32] e também com os resultados de simulações numéricas de Monte Carlo [33, 35].

Através do uso das técnicas de repesagem em histogramas múltiplos obtivemos o comportamento de gradezas físicas tais como o calor específico, energia por spin, parâmetro de ordem e a susceptibilidade do parâmetro de ordem em função da temperatura. Cannas e coautores $[33,35]$ realizaram um estudo semelhante sobre esse modelo considerando $\delta=2$ e os mesmos tamanhos de rede utilizados aqui. Dessa maneira, podemos comparar diretamente os nossos resultados com os obtidos por eles. Nossos resultados são bastante semelhantes aos encontrados por eles, porém, quantitativamente, nós obtivemos uma precisão melhor. Isso porque as técnicas de repesagem permitem cálculos das grandezas em um intervalo contínuo de temperaturas. Outro detalhe é o tempo computacional total gasto nas simulações. Quando utilizamos o algoritmo de Metropolis e não utilizamos as técnicas de repesagem, um número muito maior de séries temporais precisa ser considerado para um intervalo igual de temperaturas, o que implica em um gasto maior de tempo computacional.

Identificamos as transições de fase observando o comportamento das grandezas físicas em função da temperatura. Para a rede de tamanho $L=16$, o sistema apresenta uma única transição de fase, saindo da fase de faixas a baixas temperatura e indo para a fase de líquido tetragonal. Também identificamos a presença da fase de líquido tetragonal em todos os outros tamanhos de rede estudados. Comentamos ainda que nenhuma transição bem definida entre a fase tetragonal e a fase paramagnética para temperaturas mais altas foi encontrada nas 
simulações de Metropolis.

Observamos que o comportamento das grandezas físicas apresenta forte dependência com o tamanho da rede $L$. Um dos efeitos observados é o aparecimento de outra transição de fase para as redes maiores $(L \geq 48)$. Trata-se da transição entre a fase do tipo faixas e a fase nemática de Ising. Nossas evidências numéricas sobre essa transição têm um caráter especial, pois confirmam a previsão teórica feita por Abanov e coautores [30] e concordam com os resultados obtidos recentemente por Cannas e coautores [33]. Embora a localização das transições de fase seja fortemente dependente do tamanho da rede, a diferença entre as temperaturas críticas $T_{c}^{(2)}-T_{c}^{(1)}$ parece permanecer constante. Esses fortes efeitos de tamanho finito são relacionados com o caráter de longo alcance da interação dipolar e frustram qualquer análise simples de escala de tamanho finito. Dessa maneira, é infrutífero realizar estudos numéricos em redes pequenas, não somente por causa dos efeitos de tamanho finito, mas também porque a verdadeira fenomenologia não pode ser observada em tais tamanhos de rede. Assim, o menor tamanho de rede deve ser escolhido com cuidado e isso deve ser considerado como uma restrição importante antes de qualquer análise de tamanho finito.

Encontram-se na literatura resultados inconclusivos sobre a ordem das transições de fase, quer referente à transição faixas-tetragonal e, dependendo de $\delta$, referente às transições faixasnemática e nemática-tetragonal. Com relação à natureza da transição de fase faixas-nemática, o trabalho de Cannas e coautores [33] apresenta indícios de uma transição de fase de primeira ordem. Entretanto, ressalvas devem ser feitas devido a fatos incomuns para uma transição de primeira ordem no limite termodinâmico, como exemplo, a saturação no máximo do calor específico. Isso sugere um mecanismo do tipo Kosterlitz-Thoulosse, o que concordaria com a predição feita por Abanov e coautores [30]. Nossos resultados para a transição faixas-nemática ainda não fornecem uma indicação segura sobre a sua natureza, principalmente por causa das severas limitações nas estatísticas produzidas pelo algoritmo de Metropolis, mesmo contando com séries com $10^{7}$ varreduras. Isto nos levou ao estudo do tempo de autocorrelação para as séries produzidas por meio desse algoritmo. Com respeito à natureza da transição de fase nemática-tetragonal a situação parece pior. Cannas e coautores [33] argumentam que a transição também possui características de uma transição de primeira ordem. Mas os seus resultados são inconclusivos e atribuem este fato à presença de fortes efeitos de tamanho finito. Nossas estimativas dos expoentes críticos a partir da análise de tamanho finito indicam uma transição contínua para a transição termodinâmica nemática-tetragonal, embora nossa análise de tamanho finito ainda necessite de aprimoramento. Estudos futuros são necessários para entender a complexidade da transição faixas-nemática e para isso estamos implementando o 
algoritmo multicanônico.

Nossos cálculos para os tempos de autocorrelação integrado mostram um decaimento lento crítico mais forte em ambas as transições de fase, quando comparado com o modelo de Ising bidimensional puro. A partir desta análise, observamos estados com longos tempos de vida para temperaturas próximas ou na transição de fase faixas-nemática. Por outro lado, a transição nemática-tetragonal apresenta valores ordens de grandeza menores para $\tau_{\text {int }}$. Isso pode explicar o porque nós temos resultados melhores, no sentido de apresentar menores incertezas para as grandezas termodinâmicas, para a segunda transição.

Com respeito às simulações utilizando o algoritmo multicanônico, ressaltamos que o estudo teve um caráter exploratório e os resultados obtidos não podem ser considerados para conclusões objetivas sobre a física das transições de fase do modelo. Percebemos que um estudo mais aprofundado sobre a utilização do algoritmo multicanônico precisa ser realizado.

De qualquer maneira, notamos que a natureza da transições de fase no modelo de Ising dipolar bidimensional ainda é objeto de pesquisa na literatura. Como perspectivas futuras pretendemos estabelecer o uso do algoritmo multicanônico para exploração do sistema utilizando redes maiores. Esperamos, assim, conseguir um melhor entendimento das transições de fase presentes neste modelo. 


\section{Apêndice A}

\section{O somatório de Ewald em redes bidimensionais}

Dividimos este apêndice em cinco seções. Na primeira seção apresentamos os aspectos analíticos do método de Ewald ${ }^{1}$ e fornecemos um resultado geral para o caso em duas dimensões; na segunda mostramos dois casos particulares com importantes aplicações físicas: interações coulombianas e interações dipolares; na terceira descrevemos um método computacional para armazenar parte dos somatórios de Ewald em matrizes; na quarta apresentamos um estudo sobre a convergência numérica do método para o caso dipolar. Por último, dedicamos a quinta seção às demonstrações das relações utilizadas na primeira seção.

\section{A.1 Aspectos analíticos do método de Ewald}

Consideremos um sistema com $N$ partículas que interagem entre si através de um potencial proporcional ao inverso de uma potência da distância entre elas. Podemos definir, de maneira generalizada, a Hamiltoniana desse sistema como:

$$
\mathcal{H}^{(p)}=\frac{g}{2} \sum_{i=1}^{N} \sum_{j=1}^{N} \sum_{|\vec{n}|=0}^{\infty}, \frac{q_{i} q_{j}}{\left|\vec{r}_{i j}+\vec{n}\right|^{p}},
$$

onde $g$ define a intensidade da interação entre duas partículas $i$ e $j$ que possuem as propriedades físicas intrínsecas $q_{i}$ e $q_{j}$ (i.e., massa, carga elétrica, spin), $r_{i j}=\left|\vec{r}_{i}-\vec{r}_{j}\right|$ é a distância entre as duas partículas e $p$ um número real positivo.

O somatório infinito é incluído no potencial quando consideramos condições de contorno periódicas, onde imagens do sistema são replicadas indefinidamente em todas as direções formando uma rede (como mostra a Figura 5.1). Efetuamos o somatório sobre as componentes

\footnotetext{
${ }^{1} \mathrm{O}$ trabalho original em alemão do físico P. P. Ewald pode ser encontrado na Ref. [56]. Duas ótimas referências sobre o método são $[57,58]$.
} 
do vetor $\vec{n}$, o qual indica a posição de cada imagem. A notação ' indica que $\vec{n}=0$ é excluído do somatório quando $i=j$, impedindo a auto-interação.

A nossa apresentação do método foi principalmente inspirada na Ref. [59]. Entretanto, consideramos igualmente importante a Ref. [60], no qual o método é apresentado de forma mais geral. É necessário enfatizar que os cálculos apresentados nesta seção são válidos para qualquer dimensão espacial até a equação (A.1.13). A imposição do caráter bidimensional só aparece após esta equação, com a utilização da relação $§ 2$ deduzida na seção A.5.

Por uma questão de notação, vamos reescrever a equação (A.1.1) da maneira a seguir:

$$
\mathcal{H}^{(p)}=\frac{g}{2} \sum_{i=1}^{N} \sum_{j=1}^{N} q_{i} q_{j} S_{i j}^{(p)},
$$

com

$$
S_{i j}^{(p)}=\sum_{|\vec{n}|=0}^{\infty}, \frac{1}{\left|\vec{r}_{i j}+\vec{n}\right|^{p}} .
$$

Podemos fazer a seguinte decomposição utilizando uma função $f_{p}(\alpha r)$ :

$$
\frac{1}{r^{p}}=\frac{1-f_{p}(\alpha r)}{r^{p}}+\frac{f_{p}(\alpha r)}{r^{p}}
$$

para tanto, devemos impor os seguintes comportamentos para $f_{p}(\alpha r)$ :

i. $f_{p}(\alpha r) \rightarrow 1$ se $r \rightarrow \infty$,

ii. $f_{p}(\alpha r) \rightarrow 0$ se $r \rightarrow 0$.

O parâmetro $\alpha$ no argumento de $f_{p}$ é chamado de parâmetro de convergência de Ewald. Seu valor reflete a rapidez da convergência relativa entre as somas nos espaços real e recíproco.

A introdução da função $f_{p}(\alpha r)$ faz com que possamos reescrever a equação (A.1.2) como a soma de dois termos,

$$
S_{i j}^{(p)}=S_{i j, \text { direta }}^{(p)}+\tilde{S}_{i j}^{(p)}
$$

onde

$$
S_{i j, \text { direta }}^{(p)}=\sum_{|\vec{n}|=0}^{\infty} \frac{1-f_{p}\left(\alpha\left|\vec{r}_{i j}+\vec{n}\right|\right)}{\left|\vec{r}_{i j}+\vec{n}\right|^{p}}
$$

e

$$
\tilde{S}_{i j}^{(p)}=\sum_{|\vec{n}|=0}^{\infty}, \frac{f_{p}\left(\alpha\left|\vec{r}_{i j}+\vec{n}\right|\right)}{\left|\vec{r}_{i j}+\vec{n}\right|^{p}} .
$$

A seguir, podemos somar e subtrair o termo quando $\vec{n}=0$ e $i=j$ na equação (A.1.4), obtendo

$$
\tilde{S}_{i j}^{(p)}=S_{i j, \text { recíproco }}^{(p)}-S_{i j \text {,auto-interação }}^{(p)}
$$



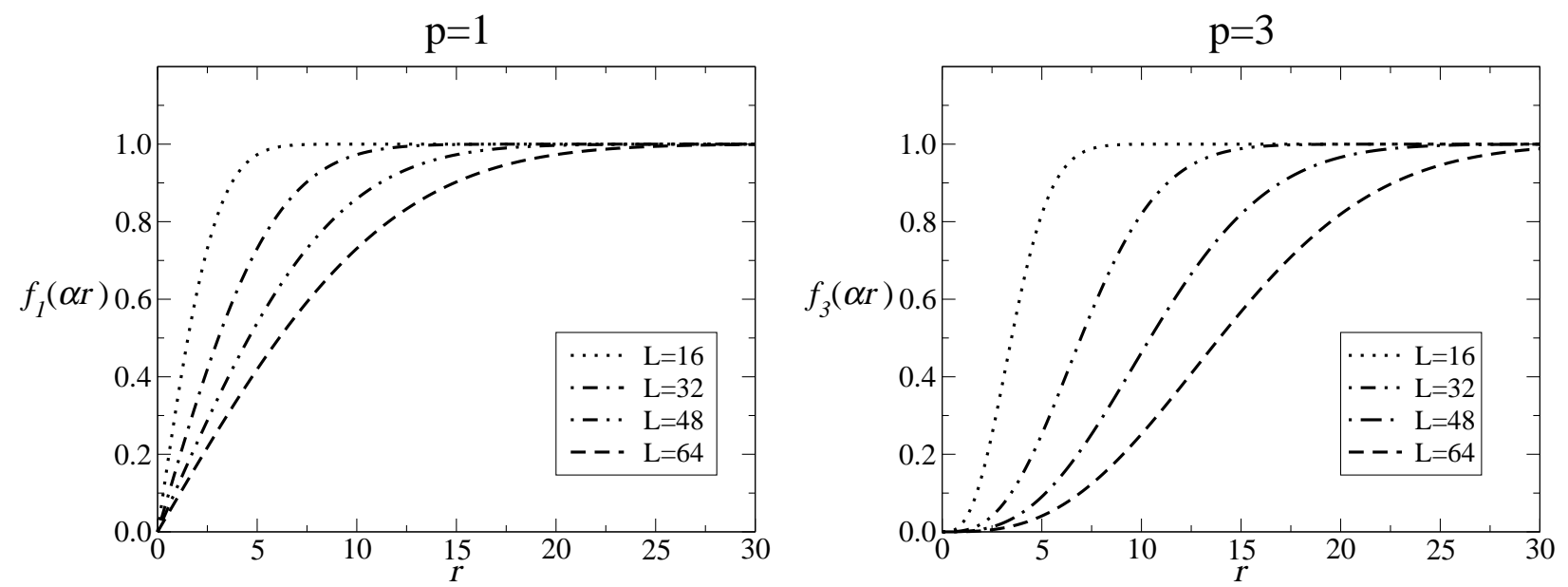

Figura A.1: Gráficos de $f_{p}(\alpha r)$ para diferentes valores de $\alpha$ e $p$, com $\alpha=5 / L$.

onde

$$
S_{i j, \text { recíproco }}^{(p)}=\sum_{|\vec{n}|=0}^{\infty} \frac{f_{p}\left(\alpha\left|\vec{r}_{i j}+\vec{n}\right|\right)}{\left|\vec{r}_{i j}+\vec{n}\right|^{p}}
$$

$\mathrm{e}$

$$
S_{i j \text {,auto-interação }}^{(p)}=\lim _{\left|\vec{r}_{i j}\right| \rightarrow 0} \frac{f_{p}\left(\alpha\left|\vec{r}_{i j}\right|\right)}{\left|\vec{r}_{i j}\right|^{p}} .
$$

Segundo as Refs. [59, 60], uma escolha adequada para a função $f_{p}(\alpha r)$ é:

$$
f_{p}(\alpha r)=\frac{\gamma\left(\frac{p}{2}, \alpha^{2} r^{2}\right)}{\Gamma\left(\frac{p}{2}\right)}
$$

onde $\Gamma\left(\frac{p}{2}\right)$ é a função gama e $\gamma\left(\frac{p}{2}, \alpha^{2} r^{2}\right)$ é a função gama incompleta inferior.

A Fig. A.1 mostra o comportamento de $f_{p}(\alpha r)$ para diferentes valores de $\alpha$ e $p$, com $\alpha=5 / L$. Mostramos, em particular, dois casos com importantes aplicações físicas: interações coulombianas $(p=1)$ e interações dipolares $(p=3)$.

Considerando a relação entre a função gama incompleta inferior com a função gama incompleta superior $\Gamma\left(\frac{p}{2}, \alpha^{2} r^{2}\right)$, conforme a Ref. [61],

$$
\Gamma(a)=\gamma(a, x)+\Gamma(a, x)
$$

podemos obter uma representação simples para $1-f_{p}(\alpha r)$,

$$
1-f_{p}(\alpha r)=\frac{\Gamma\left(\frac{p}{2}, \alpha^{2} r^{2}\right)}{\Gamma\left(\frac{p}{2}\right)} .
$$




\section{Soma no espaço real}

Inserindo o resultado apresentado em (A.1.9) na equação (A.1.3), obtemos a parte do somatório que consiste numa soma no espaço real,

$$
S_{i j \text {, real }}^{(p)}=\frac{1}{\Gamma\left(\frac{p}{2}\right)} \sum_{|\vec{n}|=0}^{\infty}, \frac{\Gamma\left(\frac{p}{2}, \alpha^{2}\left|\vec{r}_{i j}+\vec{n}\right|^{2}\right)}{\left|\vec{r}_{i j}+\vec{n}\right|^{p}} .
$$

\section{Soma no espaço recíproco}

De maneira análoga, podemos substituir a definição introduzida em (A.1.7) na equação (A.1.5) para obter

$$
S_{i j \text {,recíproco }}^{(p)}=\frac{1}{\Gamma\left(\frac{p}{2}\right)} \sum_{|\vec{n}|=0}^{\infty} \frac{\gamma\left(\frac{p}{2}, \alpha^{2}\left|\vec{r}_{i j}+\vec{n}\right|^{2}\right)}{\left|\vec{r}_{i j}+\vec{n}\right|^{p}} .
$$

Fazendo uso da distribuição delta de Dirac podemos reescrever a equação (A.1.11) da seguinte forma:

$$
S_{i j \text {,recíproco }}^{(p)}=\frac{1}{\Gamma\left(\frac{p}{2}\right)} \int \sum_{|\vec{n}|=0}^{\infty} \delta\left(\vec{r}-\left(\vec{r}_{i j}+\vec{n}\right)\right) \frac{\gamma\left(\frac{p}{2}, \alpha^{2}|\vec{r}|^{2}\right)}{|\vec{r}|^{p}} d \vec{r} .
$$

Agora, considerando a relação abaixo, demonstrada em A.5.1,

$$
\sum_{|\vec{n}|=0}^{\infty} \delta\left(\vec{r}-\left(\vec{r}_{i j}+\vec{n}\right)\right)=\frac{1}{V_{d}} \sum_{|\vec{G}|=0}^{\infty} e^{i \vec{G} \cdot\left(\vec{r}-\vec{r}_{i j}\right)}
$$

onde $V_{d}=L^{d}, L$ é o comprimento do sistema, $d$ é a dimensão espacial e o vetor $\vec{G}$ é um vetor no espaço recíproco, podemos reescrever a equação (A.1.12) como segue,

$$
S_{i j \text {,recíproco }}^{(p)}=\frac{1}{V_{d} \Gamma\left(\frac{p}{2}\right)} \sum_{|\vec{G}|=0}^{\infty}\left[\int \frac{\gamma\left(\frac{p}{2}, \alpha^{2}|\vec{r}|^{2}\right)}{|\vec{r}|^{p}} e^{i \vec{G} \cdot \vec{r}} d \vec{r}\right] e^{-i \vec{G} \cdot \vec{r}_{i j}}
$$

Podemos utilizar a relação a seguir

$$
\int \frac{\gamma\left(\alpha^{2}|\vec{r}|^{2}\right)}{|\vec{r}|^{p}} e^{i \vec{G} \cdot \vec{r}} d^{2} \vec{r}=\pi \frac{G^{p-2}}{2^{p-2}} \Gamma\left(-\frac{p}{2}+1, \frac{G^{2}}{4 \alpha^{2}}\right)
$$

para calcular a integral dentro do parênteses em (A.1.13). Desta forma obtemos a expressão para a parte recíproca do somatório de Ewald:

$$
S_{i j \text {,recíproco }}^{(p)}=\frac{\pi}{L^{2} \Gamma\left(\frac{p}{2}\right)} \sum_{|\vec{G}|=0}^{\infty} \frac{G^{p-2}}{2^{p-2}} \Gamma\left(-\frac{p}{2}+1, \frac{G^{2}}{4 \alpha^{2}}\right) e^{-i \vec{G} \cdot \vec{r}_{i j}} .
$$




\section{Termo de auto-interação}

Por último, substituindo a definição (A.1.7) na expressão (A.1.6) obtemos

$$
S_{i j, \text { auto-interação }}^{(p)}=\frac{1}{\Gamma\left(\frac{p}{2}\right)} \lim _{r_{i j} \rightarrow 0} \frac{\gamma\left(\frac{p}{2}, \alpha^{2} r_{i j}^{2}\right)}{r_{i j}^{p}} .
$$

Fazendo a mudança de variável $x=\alpha^{2} r_{i j}^{2}$ e observando que:

$$
\begin{aligned}
& \text { i. } \lim _{r_{i j} \rightarrow 0} \gamma\left(\frac{p}{2}, \alpha^{2} r_{i j}^{2}\right)=\lim _{x \rightarrow 0} \gamma\left(\frac{p}{2}, x\right)=0, \\
& \text { ii. } \lim _{r_{i j} \rightarrow 0} r_{i j}^{p}=\lim _{x \rightarrow 0} \frac{x^{\frac{p}{2}}}{\alpha^{p}}=0,
\end{aligned}
$$

podemos calcular o limite na equação (A.1.15) através da regra de L'Hopital. Assim,

$$
S_{i j, \text { auto-interação }}^{(p)}=\frac{1}{\Gamma\left(\frac{p}{2}\right)} \lim _{x \rightarrow 0}\left[\alpha^{p} \frac{\frac{d}{d x} \gamma\left(\frac{p}{2}, x\right)}{\frac{d}{d x} x^{\frac{p}{2}}}\right] .
$$

Sendo (Ref. [61]):

$$
\frac{d}{d x} \gamma\left(\frac{p}{2}, x\right)=x^{\frac{p}{2}-1} e^{-x}
$$

e

$$
\frac{d}{d x} x^{\frac{p}{2}}=\frac{p}{2} x^{\frac{p}{2}-1}
$$

obtemos:

$$
\lim _{x \rightarrow 0} \alpha^{p} \frac{\frac{d}{d x} \gamma\left(\frac{p}{2}, x\right)}{\frac{d}{d x} x^{\frac{p}{2}}}=\frac{2 \alpha^{p}}{p} \lim _{x \rightarrow 0} \frac{x^{\frac{p}{2}-1} e^{-x}}{x^{\frac{p}{2}-1}}=\frac{2 \alpha^{p}}{p} \lim _{x \rightarrow 0} e^{-x}=\frac{2 \alpha^{p}}{p} .
$$

Note que fazer $r_{i j} \rightarrow 0$ (ou $x \rightarrow 0$ ) equivale a fazer $i=j$. Substituindo o resultado acima na expressão (A.1.16) e incluindo a delta de Kronecker, obtemos a expressão da parte de auto-interação do somatório:

$$
S_{i j, \text { auto-interação }}^{(p)}=\delta_{i j} \frac{2 \alpha^{p}}{p \Gamma\left(\frac{p}{2}\right)} .
$$

\section{Resultado geral}

Utilizando as expressões (A.1.10), (A.1.14) e (A.1.19), obtemos uma expressão geral para as somas como segue:

$$
S_{i j}^{(p)}=\frac{1}{\Gamma\left(\frac{p}{2}\right)}\left[\sum_{|\vec{n}|=0}^{\infty} \frac{\Gamma\left(\frac{p}{2}, \alpha^{2}\left|\vec{r}_{i j}+\vec{n}\right|^{2}\right)}{\left|\vec{r}_{i j}+\vec{n}\right|^{p}}+\frac{\pi}{L^{2}} \sum_{|\vec{G}|=0}^{\infty} \frac{G^{p-2}}{2^{p-2}} \Gamma\left(-\frac{p}{2}+1, \frac{G^{2}}{4 \alpha^{2}}\right) e^{-i \vec{G} \cdot \vec{r}_{i j}}-\delta_{i j} \frac{2 \alpha^{p}}{p}\right] .
$$


Finalmente, considerando a relação (A.5.3)

$$
\sum_{|\vec{G}|=0}^{\infty} \mathcal{F}(G) e^{-i \vec{G} \cdot \vec{r}}=\sum_{|\vec{G}|=0}^{\infty} \mathcal{F}(G) \cos (\vec{G} \cdot \vec{r})
$$

obtemos uma expressão geral para o somatório de Ewald para um sistema bidimensional:

$$
S_{i j}^{(p)}=\frac{1}{\Gamma\left(\frac{p}{2}\right)}\left[\sum_{|\vec{n}|=0}^{\infty} \frac{\Gamma\left(\frac{p}{2}, \alpha^{2}\left|\vec{r}_{i j}+\vec{n}\right|^{2}\right)}{\left|\vec{r}_{i j}+\vec{n}\right|^{p}}+\frac{\pi}{L^{2}} \sum_{|\vec{G}|=0}^{\infty} \frac{G^{p-2}}{2^{p-2}} \Gamma\left(-\frac{p}{2}+1, \frac{G^{2}}{4 \alpha^{2}}\right) \cos \left(\vec{G} \cdot \vec{r}_{i j}\right)-\delta_{i j} \frac{2 \alpha^{p}}{p}\right] .
$$

\section{A.2 Casos particulares}

Cálculos de energias da cristais iônicos e considerações sobre a estabilidade de diversos tipos de rede são os exemplos mais velhos e mais conhecidos que utilizam a técnica de soma de Ewald. Outros casos onde os cálculos dessas somas têm importância são as investigações de propriedades elétricas, ópticas, ou elásticas, de cristais. A seguir apresentamos dois casos particulares que são bastante comuns na Física: o caso das interações de Coulomb e o caso das interações do tipo dipolo (elétrico ou magnético).

\section{A.2.1 Interação de Coulomb}

A interação entre duas cargas elétricas pontuais é determinada através do potencial de Coulomb. Como exemplo clássico onde interações de Coulomb são consideradas temos o cálculo da constante de Madelung, a qual corresponde à energia eletrostática de uma rede iônica cristalina. Para obter o cálculo das interações de Coulomb em um dado sistema, temos que utilizar a expressão (A.1.21) considerando $p=1$. Nesse caso, teremos

$$
\begin{aligned}
\frac{\Gamma\left(\frac{1}{2}, \alpha^{2}\left|\vec{r}_{i j}+\vec{n}\right|^{2}\right)}{\Gamma\left(\frac{1}{2}\right)} & =\operatorname{erfc}\left(\alpha\left|\vec{r}_{i j}+\vec{n}\right|\right) \\
\frac{\Gamma\left(\frac{1}{2}, \frac{G^{2}}{4 \alpha^{2}}\right)}{\Gamma\left(\frac{1}{2}\right)} & =\operatorname{erfc}\left(\frac{G}{2 \alpha}\right)
\end{aligned}
$$

e $g=1 / 4 \pi \varepsilon, \operatorname{assim} q_{i}$ e $q_{j}$ representarão as cargas elétricas das partículas. Assim, a energia eletrostática de interação, escrita utilizando a técnica de soma de Ewald, de uma rede cristalina 
composta de $N$ cargas elétricas, será dada por:

$$
\begin{aligned}
E_{\text {Coulomb }}^{(1)}= & \frac{1}{2} \sum_{i, j=1}^{N} \sum_{|\vec{n}|=0}^{\infty}{ }^{\prime} q_{i} q_{j} \frac{\operatorname{erfc}\left(\alpha\left|\vec{r}_{i j}+\vec{n}\right|\right)}{\left|\vec{r}_{i j}+\vec{n}\right|} \\
& +\frac{\pi}{L^{2}} \sum_{i, j=1}^{N} \sum_{|\vec{G}| \neq 0}^{\infty} q_{i} q_{j} \frac{\operatorname{erfc}\left(\frac{G}{2 \alpha}\right) \cos \left(\vec{G} \cdot \vec{r}_{i j}\right)}{G} \\
& -\frac{\alpha}{\sqrt{\pi}} \sum_{i=1}^{N} q_{i}^{2} .
\end{aligned}
$$

Nesse caso, vale notar que, interações que diminuem lentamente com a distância, como é o caso da interação de Coulomb, podem dar origem a somas sobre a rede as quais exibem somente uma convergência condicional. Desde que nesse caso a soma das séries não é determinada unicamente, argumentos físicos (como a neutralidade da rede, $\sum_{i} q_{i}=0$ ) têm que ser invocados no sentido de obter-se respostas que apresentem algum sentido físico.

\section{A.2.2 Interação dipolar}

O caso particular $p=3$ corresponde ao potencial devido às interações do tipo dipolo. Nessa dissertação consideramos a interação entre momentos de dipolo dos spins num sistema magnético com spins de Ising, porém, as interações do tipo dipolo também são encontradas em sistemas com cargas elétricas. Para determinar a energia de interação total entre os momentos de dipolo em um sistema com $N$ partículas precisamos substituir $p=3$ na expressão (A.1.21). Dessa maneira, obtemos a seguinte expressão:

$$
\begin{aligned}
E_{\text {dipolar }}^{(3)}= & \frac{g}{2} \sum_{i=1}^{N} \sum_{j=1}^{N} \sum_{|\vec{n}|=0}^{\infty}{ }^{\prime} \frac{\sigma_{i} \sigma_{j}}{\left|\vec{r}_{i j}+\vec{n}\right|^{3}}\left[\frac{\Gamma\left(\frac{3}{2}, \alpha^{2}\left|\vec{r}_{i j}+\vec{n}\right|^{2}\right)}{\Gamma\left(\frac{3}{2}\right)}\right] \\
& +g \frac{\pi}{2 L^{2}} \sum_{i=1}^{N} \sum_{j=1}^{N} \sum_{|\vec{G}|=0}^{\infty} \sigma_{i} \sigma_{j} \frac{G}{2}\left[\frac{\Gamma\left(-\frac{1}{2}, \frac{G^{2}}{4 \alpha^{2}}\right)}{\Gamma\left(\frac{3}{2}\right)}\right] \cos \left(\vec{G} \cdot \vec{r}_{i j}\right) \\
& -g \frac{\alpha^{3}}{3 \Gamma\left(\frac{3}{2}\right)} \sum_{i=1}^{N} \sigma_{i}^{2} .
\end{aligned}
$$

onde $q_{i}$ representará o momento de dipolo elétrico, no caso de um sistema composto de cargas, ou o momento de dipolo magnético, no caso de um sistema magnético.

Utilizando a relação de recorrência (vide Ref. [61]) para função gama incompleta superior,

$$
\Gamma(a+1, x)=a \Gamma(a, x)+x^{a} e^{-x}
$$

obtemos

$$
\frac{\Gamma\left(\frac{3}{2}, \alpha^{2}\left|\vec{r}_{i j}+\vec{n}\right|^{2}\right)}{\Gamma\left(\frac{3}{2}\right)}=\frac{1}{\Gamma\left(\frac{3}{2}\right)}\left[\frac{1}{2} \Gamma\left(\frac{1}{2}, \alpha^{2}\left|\vec{r}_{i j}+\vec{n}\right|^{2}\right)+\alpha\left|\vec{r}_{i j}+\vec{n}\right| e^{-\alpha^{2}\left|\vec{r}_{i j}+\vec{n}\right|^{2}}\right] .
$$


Invertendo a relação (A.2.4) temos,

$$
\Gamma(a, x)=\frac{1}{a}\left[\Gamma(a+1, x)-x^{a} e^{-x}\right] .
$$

Utilizando a relação acima obtemos

$$
\frac{\Gamma\left(-\frac{1}{2}, \frac{G^{2}}{4 \alpha^{2}}\right)}{\Gamma\left(\frac{3}{2}\right)}=-\frac{2}{\Gamma\left(\frac{3}{2}\right)}\left[\Gamma\left(\frac{1}{2}, \frac{G^{2}}{4 \alpha^{2}}\right)-\left(\frac{G}{2 \alpha}\right)^{-1} e^{-\frac{G^{2}}{4 \alpha^{2}}}\right] .
$$

Considerando os resultados particulares,

$$
\Gamma\left(\frac{3}{2}\right)=\frac{\sqrt{\pi}}{2}
$$

e

$$
\Gamma\left(\frac{1}{2}, \alpha^{2} r^{2}\right)=\sqrt{\pi} \operatorname{erfc}(\alpha r)
$$

reescrevemos as expressões (A.2.5) e (A.2.7), respectivamente, como

$$
\begin{aligned}
\frac{\Gamma\left(\frac{3}{2}, \alpha^{2}\left|\vec{r}_{i j}+\vec{n}\right|^{2}\right)}{\Gamma\left(\frac{3}{2}\right)} & =\operatorname{erfc}\left(\alpha\left|\vec{r}_{i j}+\vec{n}\right|\right)+\frac{2 \alpha}{\sqrt{\pi}}\left|\vec{r}_{i j}+\vec{n}\right| e^{-\alpha^{2}\left|\vec{r}_{i j}+\vec{n}\right|^{2}} \\
\frac{\Gamma\left(-\frac{1}{2}, \frac{G^{2}}{4 \alpha^{2}}\right)}{\Gamma\left(\frac{3}{2}\right)} & =-4\left[\operatorname{erfc}\left(\frac{G}{2 \alpha}\right)-\frac{2 \alpha}{\sqrt{\pi}} \frac{e^{-\frac{G^{2}}{4 \alpha^{2}}}}{G}\right]
\end{aligned}
$$

Finalmente, substituindo (A.2.8) e (A.2.9) em (A.2.3) obtemos:

$$
\begin{aligned}
& E_{\text {dipolar }}^{(3)}=\frac{g}{2} \sum_{i=1}^{N} \sum_{j=1}^{N} \sum_{|\vec{n}|=0}^{\infty}{ }^{\prime} \frac{\sigma_{i} \sigma_{j}}{\left|\vec{r}_{i j}+\vec{n}\right|^{3}}\left[\operatorname{erfc}\left(\alpha\left|\vec{r}_{i j}+\vec{n}\right|\right)+\frac{2 \alpha}{\sqrt{\pi}}\left|\vec{r}_{i j}+\vec{n}\right| e^{-\alpha^{2}\left|\vec{r}_{i j}+\vec{n}\right|^{2}}\right] \\
& +g \frac{\pi}{L^{2}} \sum_{i=1}^{N} \sum_{j=1}^{N} \sum_{|\vec{G}|=0}^{\infty} \sigma_{i} \sigma_{j}\left[\frac{2 \alpha}{\sqrt{\pi}} e^{-\frac{G^{2}}{4 \alpha^{2}}}-G \operatorname{erfc}\left(\frac{G}{2 \alpha}\right)\right] \cos \left(\vec{G} \cdot \vec{r}_{i j}\right)-g \frac{2 \alpha^{3}}{3 \sqrt{\pi}} \sum_{i=1}^{N} \sigma_{i}^{2}
\end{aligned}
$$

\section{A.3 Método de armazenamento}

O uso de matrizes para armazenar parte dos somatórios oriundos do método de Ewald evita que muitas operações repetitivas sejam efetuadas no cálculo da energia de interação, aumentando, assim, a eficiência dos algoritmos que envolvem esse cálculo.

Enfatizamos nesta seção alguns conceitos práticos afim de facilitar tanto a compreensão quanto a implementação do método.

É importante ressaltar que a discussão feita aqui limita-se ao caso onde todas as partículas do sistema estão posicionadas sobre uma rede regular bidimensional (quadrada de lado $L$ ) e o potencial de interação entre elas é do tipo dipolo, no entanto, é possível utilizar este método 
em outros casos. Poderíamos, por exemplo, considerar moléculas que constituem um líquido interagindo através de um potencial coulombiano. Neste caso, onde as posições espaciais correspondem a variáveis contínuas, poderíamos utilizar os valores armazenados de potenciais sobre a rede para obter, por meio de interpolação, o valor de potenciais sobre moléculas que não estão localizadas nos sítios de uma rede (vide Ref. [62]).

Comecemos reescrevendo a parte real de $E_{\text {dipolar }}^{(3)}$ da seguinte maneira:

$$
E_{\text {real }}=\sum_{i=1}^{N} \sum_{j=1}^{N} \sum_{|\vec{n}|=0}^{\infty}{ }^{\prime} \sigma_{i} \sigma_{j} A_{i j, \vec{n}} .
$$

Para o caso de interações dipolares temos explicitamente

$$
A_{i j, \vec{n}}=\frac{1}{2}\left[\frac{\operatorname{erfc}\left(\alpha\left|\vec{r}_{i j}+\vec{n}\right|\right)}{\left|\vec{r}_{i j}+\vec{n}\right|^{3}}+\frac{2 \alpha}{\sqrt{\pi}} \frac{e^{-\alpha^{2}\left|\vec{r}_{i j}+\vec{n}\right|^{2}}}{\left|\vec{r}_{i j}+\vec{n}\right|^{2}}\right] .
$$

Notamos que $A_{i j, \vec{n}}$ representa o potencial de interação entre uma partícula $i$ e a imagem, determinada por $\vec{n}$, da partícula $j$, e que o seu valor depende apenas do módulo do vetor $\vec{r}_{i j}+\vec{n}$.

Representando os vetores posição $\vec{r}_{i}$ e $\vec{r}_{j}$ pelos pares de coordenadas cartesianas $\left(i_{1}, i_{2}\right)$ e $\left(j_{1}, j_{2}\right)$, respectivamente, e o vetor $\vec{n}$ pelo par cartesiano $\left(n_{1} L, n_{2} L\right)$, podemos reescrever $\left|\vec{r}_{i j}+\vec{n}\right|$ como

$$
\left|\vec{r}_{i j}+\vec{n}\right|=\sqrt{\left(i_{1}-j_{1}+n_{1} L\right)^{2}+\left(i_{2}-j_{2}+n_{2} L\right)^{2}},
$$

onde $\left\{i_{1}, i_{2}, j_{1}, j_{2} \in \mathbf{N} \mid 1 \leq i_{1}, i_{2}, j_{1}, j_{2} \leq L\right\}$ para partículas localizadas sobre a rede e $\left\{n_{1}, n_{2} \in \mathbf{Z} \mid-\infty<n_{1}, n_{2}<\infty\right\}$. As coordenadas das partículas assumem valores inteiros pois definimos o parâmetro de rede $a$, o qual determina a distância mínima entre duas partículas na rede, igual a $\mathrm{um}^{2}$.

Fazendo a seguinte mudança de variáveis

$$
l=i_{1}-j_{1}+n_{1} L \quad \text { e } \quad m=i_{2}-j_{2}+n_{2} L,
$$

podemos reescrever $\left|\vec{r}_{i j}+\vec{n}\right|$ em termos de $l$ e $m$ como:

$$
r_{l m}=\sqrt{l^{2}+m^{2}}
$$

onde $l, m \in(-\infty, \infty)$ e $r_{l m} \in[0, \infty)$.

Dessa maneira, podemos "mapear" $A_{i j, \vec{n}}=A_{\left(i_{1}, i_{2}\right),\left(j_{1}, j_{2}\right),\left(n_{1}, n_{2}\right)}$ em $A_{l m}$, que é dado por:

$$
A_{l m}=\frac{1}{2}\left[\frac{\operatorname{erfc}\left(\alpha r_{l m}\right)}{r_{l m}^{3}}+\frac{2 \alpha}{\sqrt{\pi}} \frac{e^{-\alpha^{2} r_{l m}^{2}}}{r_{l m}^{2}}\right] \text {. }
$$




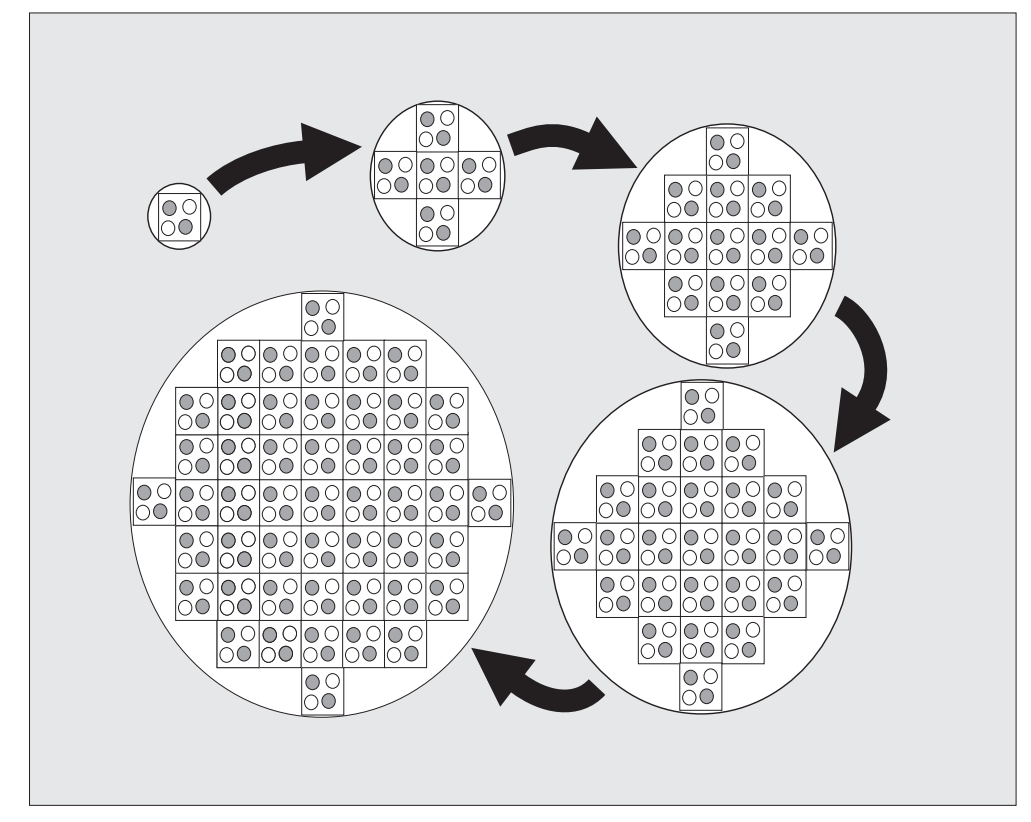

Figura A.2: Representação do modo convencional de efetuar os somatórios infinitos sobre as componentes do vetor $\vec{n}$ na expressão (A.3.1). Figura extraída de [48].

A Figura A.2 mostra o modo convencional de efetuarmos os somatórios infinitos sobre as componentes do vetor $\vec{n}$ na expressão (A.3.1). Primeiro somamos os termos para $\vec{n}=0$, depois para $0<|\vec{n}| \leq 1$, depois para $1<|\vec{n}| \leq 2$, e assim por diante. Na nossa notação, $r_{l m}$ representa a distância entre uma partícula $i$ e uma partícula $j$ (ou uma imagem de $j$ ), com isso, efetuar os somatórios de acordo com o modo convencional significa somar primeiro os termos para $r_{l m}$ pequena, ou nula ${ }^{3}$. A princípio, a distância $r_{l m}$ pode ser infinita, porém, como

$$
\lim _{r_{l m} \rightarrow \infty} \frac{\operatorname{erfc}\left(\alpha r_{l m}\right)}{r_{l m}^{3}}=0 \quad \text { e } \quad \lim _{r_{l m} \rightarrow \infty} \frac{e^{-\alpha^{2} r_{l m}^{2}}}{r_{l m}^{2}}=0,
$$

percebemos que $A_{l m} \rightarrow 0$ para $r_{l m} \rightarrow \infty$, então os somatórios em (A.3.1) convergem.

É evidente que, numericamente, não é conveniente trabalhar com um número muito grande de termos nos somatórios, dessa forma, determinamos um limite superior para $r_{l m}$. Por conveniência, fazemos isso definindo o parâmetro $r_{\max }$, o qual determina arbitrariamente o módulo máximo das variáveis $l$ e $m$, e, com isso, $r_{l m} \in\left[0, r_{\max } \sqrt{2}\right]$.

Podemos então criar uma matriz $A$, de tamanho $2 r_{\max }+1 \times 2 r_{\max }+1$, cujos elementos são dados por $A_{l m}, \operatorname{com} l, m \in\left[-r_{\max }, r_{\max }\right]$. Contudo, devido à simetria, o valor de $A_{l m}$ fica inalterado para qualquer combinação de $l$ e $m$, seja ela $(l, m),(l,-m),(-l, m),(-l,-m)$,

\footnotetext{
${ }^{2}$ Fixamos o parâmetro de rede igual a um pois altera-lo equivale a alterar o acoplamento $g$.

${ }^{3}$ Lembramos que o elemento de auto-interação $(i=j$ e $\vec{n}=0)$ não deve ser considerado, assim, de forma arbitrária, fazemos $A_{00}=0$.
} 
$(m, l),(m,-l),(-m, l)$ ou $(-m,-l)$. Dessa maneira redefinimos o tamanho da matriz $A$ para $r_{\max }+1 \times r_{\max }+1$, com os elementos dados por $A_{|l|,|m|}$.

$\mathrm{Na}$ prática, ao invés de efetuar os somatórios

$$
E_{\text {real }}=\sum_{i_{1}=1}^{L} \sum_{i_{2}=1}^{L} \sum_{j_{1}=1}^{L} \sum_{j_{2}=1}^{L} \sum_{n_{1}=-\infty}^{\infty}, \sum_{n_{2}=-\infty}^{\infty}{ }^{\prime} \sigma_{\left(i_{1}, i_{2}\right)} \sigma_{\left(j_{1}, j_{2}\right)} A_{\left(i_{1}, i_{2}\right),\left(j_{1}, j_{2}\right),\left(n_{1}, n_{2}\right)}
$$

efetuamos

$$
E_{\text {real }}=\sum_{i_{1}=1}^{L} \sum_{i_{2}=1}^{L} \sum_{l=-r_{\max }}^{r_{\max }} \sum_{m=-r_{\max }}^{r_{\max }} \sigma_{\left(i_{1}, i_{2}\right)} \sigma_{\left(j_{1}, j_{2}\right)} A_{|l|,|m|}
$$

onde

$$
j_{1}=l+i_{1}-n_{1} L \quad \text { e } \quad j_{2}=m+i_{2}-n_{2} L
$$

respeitando a condição $1 \leq j_{1}, j_{2} \leq L$. Respeitar essa condição equivale a, por exemplo, considerar $n_{1} \geq 1$ se $l+i_{1} \geq L$, ou considerar $n_{2} \leq 1$ se $m+i_{2} \leq L$. Ou seja, equivale a respeitar as condições de contorno periódicas.

Vamos considerar agora a parte recíproca do Hamiltoniano. Podemos escrevê-la da seguinte maneira:

$$
E_{\text {recíproco }}=\sum_{i=1}^{N} \sum_{j=1}^{N} \sigma_{i} \sigma_{j} B_{i j}
$$

Comparando com a parte recíproca do Hamiltoniano dada pela expressão (A.2.10), percebemos que os somatórios infinitos sobre as componentes do vetor $\vec{G}$ foram inseridos em $B_{i j}$. A vantagem é que, com isso, podemos definir uma matriz $B$ cujos elementos são dados por $B_{i j}$, com $1 \leq i, j \leq N$.

Para o caso de interações dipolares temos os elementos $B_{i j}$ dados explicitamente por:

$$
B_{i j}=\frac{\pi}{L^{2}} \sum_{|\vec{G}|=0}^{\infty}\left[\frac{2 \alpha}{\sqrt{\pi}} e^{-\frac{G^{2}}{4 \alpha^{2}}}-G \operatorname{erfc}\left(\frac{G}{2 \alpha}\right)\right] \cos \left(\vec{G} \cdot \vec{r}_{i j}\right)
$$

Substituindo o vetor $\vec{G}=\frac{2 \pi}{L}\left(k_{1} \hat{e}_{1}+k_{2} \hat{e}_{2}\right)$ na expressão acima obtemos:

$$
\begin{array}{r}
B_{i j}=\frac{\pi}{L^{2}} \sum_{k_{1}=-\infty}^{\infty} \sum_{k_{2}=-\infty}^{\infty}\left[\frac{2 \alpha}{\sqrt{\pi}} e^{-\frac{k_{1}^{2}+k_{2}^{2}}{\pi^{2} \alpha^{2}}}-\frac{2 \pi \sqrt{k_{1}^{2}+k_{2}^{2}}}{L} \operatorname{erfc}\left(\frac{\sqrt{k_{1}^{2}+k_{2}^{2}}}{\pi \alpha}\right)\right] \\
\cos \left[\frac{2 \pi}{L}\left(k_{1} \hat{e}_{1}+k_{2} \hat{e}_{2}\right)\left(\vec{r}_{i}-\vec{r}_{j}\right)\right] .
\end{array}
$$

De maneira análoga à parte real, podemos representar os vetores posição $\vec{r}_{i}$ e $\vec{r}_{j}$ através de pares cartesianos, assim teremos:

$$
\cos \left[\frac{2 \pi}{L}\left(k_{1} \hat{e}_{1}+k_{2} \hat{e}_{2}\right)\left(\vec{r}_{i}-\vec{r}_{j}\right)\right]=\cos \left\{\frac{2 \pi}{L}\left[k_{1}\left(i_{1}-j_{1}\right)+k_{2}\left(i_{2}-j_{2}\right)\right]\right\} .
$$


Fazendo a mudança de variáveis:

$$
l=i_{1}-j_{1} \quad \text { e } \quad m=i_{2}-j_{2},
$$

obtemos

$$
\cos \left[\frac{2 \pi}{L}\left(k_{1} l+k_{2} m\right)\right]
$$

onde $-(L-1) \leq l, m \leq L-1$.

Dessa forma, os elementos $B_{i j}=B_{\left(i_{1}, i_{2}\right),\left(j_{1}, j_{2}\right)}$ podem ser "mapeados" em elementos $B_{l m}$. Redefinimos então o tamanho da matriz $B$ para $2 L+1 \times 2 L+1$, com elementos dados por:

$$
B_{l m}=\frac{\pi}{L^{2}} \sum_{k_{1}=-\infty}^{\infty} \sum_{k_{2}=-\infty}^{\infty}\left[\frac{2 \alpha}{\sqrt{\pi}} e^{-\frac{k_{1}^{2}+k_{2}^{2}}{\pi^{2} \alpha^{2}}}-\frac{2 \pi \sqrt{k_{1}^{2}+k_{2}^{2}}}{L} \operatorname{erfc}\left(\frac{\sqrt{k_{1}^{2}+k_{2}^{2}}}{\pi \alpha}\right)\right] \cos \left[\frac{2 \pi}{L}\left(k_{1} l+k_{2} m\right)\right]
$$

Note que, devido a paridade da função cosseno e a simetria dos valores assumidos por $k_{1}$ e $k_{2}$ nos somatórios, o valor de $B_{l m}$ fica inalterado para qualquer combinação de $l$ e $m$, ou seja, $B_{l m}=B_{|l|,|m|}$. Com isso, redefinimos novamente o tamanho da matriz $B$ para $L+1 \times L+1$.

Considerando os limites

$$
\lim _{k \rightarrow \infty} e^{-\frac{k^{2}}{\pi^{2} \alpha^{2}}}=0 \quad \text { e } \quad \lim _{k \rightarrow \infty} \frac{2 \pi k}{L} \operatorname{erfc}\left(\frac{k}{\pi \alpha}\right)=0,
$$

com $k=\sqrt{k_{1}^{2}+k_{2}^{2}}$; e a propriedade da função cosseno: $|\cos (x)| \leq 1 \quad \forall x \in \Re$; percebemos que, termos para os quais $k_{1}$ e $k_{2}$ são muito grandes não contribuem para o valor de $B_{l m}$ (independente dos valores de $l$ e $m$ ). Assim, ao invés de considerarmos uma infinidade de termos nos somatórios na expressão (A.3.4), definimos o parâmetro $k_{\max }$, o qual determina o limite superior para o módulo das variáveis $k_{1}$ e $k_{2}$. Na prática, efetuamos:

$$
E_{\text {recíproco }}=\sum_{i=1}^{N} \sum_{j=1}^{N} \sigma_{i} \sigma_{j} B_{|l|,|m|} \text {. }
$$

Notamos que o termo de auto-interação do Hamiltoniano não é alterado seja qual for a configuração do sistema, assim, não precisamos de matrizes para armazenar o seu valor.

Por fim, observamos a presença do parâmetro $\alpha$ tanto nos elementos $A_{l m}$ definidos pela expressão (A.3.2) quanto nos elementos $B_{l m}$ definidos pela expressão (A.3.4). Por um lado, quando atribuimos um valor grande ao parâmetro $\alpha$, notamos que somente valores pequenos de $r_{l m}$ fornecem valores consideráveis aos elementos $A_{l m}$. Com isso, percebemos que poucos termos precisam ser considerados nos somatórios da expressão (A.3.3) para que $E_{\text {real }}$ convirja, ou seja, podemos fazer $r_{\text {max }}$ pequeno. Por outro lado, fazendo $\alpha$ grande, percebemos que mais termos são necessários nos somatórios da expressão (A.3.4) para que $E_{\text {recíproco }}$ convirja, ou seja, precisamos de um $k_{\max }$ grande. 


\section{A.4 Estudo numérico da convergência dos somatórios}

Conforme discutido na seção anterior, existe uma relação entre o valor que atribuimos ao parâmetro $\alpha$, também chamado de parâmetro de convergência de Ewald, e os valores que atribuimos aos parâmetros $r_{\max }$ e $k_{\max }$. Vimos que os parâmetros $r_{\max }$ e $k_{\max }$, por sua vez, determinam o número de termos que devem ser considerados nas somas real e recíproca, respectivamente. O fato de consideramos um número finito termos nas somas implica em erros numéricos. Tendo isso em vista, realizamos um estudo numérico da convergência dos valores de $E_{\text {real }}$ e $E_{\text {recíproco }}$ com intuito de minimizar o erro numérico cometido no cálculo da energia de interação dipolar.

Para este estudo numérico consideramos um sistema com $N=L^{2}$ spins e com a condição, por exemplo, $\sigma_{i}=1(i=1, \ldots, N)$. Calculamos $E_{\text {real }}$, definido pela expressão (A.3.3), e

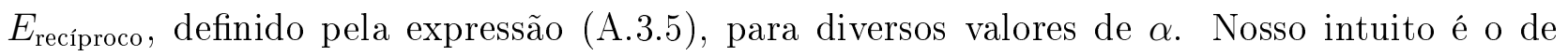
estimar os valores dos parâmetros $r_{\max }$ e $k_{\max }$ para um erro absoluto, dado pela expressão (A.4.1), de $10^{-10}$. Os resultados são apresentados nas tabelas abaixo para diversos tamanhos de rede $L$.

$$
\varepsilon\left(r_{\max }, k_{\max }\right)=\varepsilon_{\text {real }}\left(r_{\max }\right)+\varepsilon_{\text {recíproco }}\left(k_{\max }\right)
$$

onde

$$
\varepsilon_{\text {real }}\left(r_{\text {max }}\right)=\left|E_{\text {real }}^{*}-E_{\text {real }}\left(r_{\text {max }}\right)\right|
$$

e

$$
\varepsilon_{\text {recíproco }}\left(k_{\max }\right)=\left|E_{\text {recíproco }}^{*}-E_{\text {recíproco }}\left(k_{\max }\right)\right| \text {. }
$$

Sendo $E_{\text {real }}^{*}$ definido com $r_{\text {max }}$ muito grande e $E_{\text {recíproco }}^{*}$ definido com $k_{\text {max }}$ muito grande.

Utilizando os valores das Tabelas A.1, A.2, A.3, A.4 e A.5 calculamos os valores de $E_{\text {dipolar }} / N$ para diversos valores de $\alpha$ e de $N$, considerando um sistema com configuração $\sigma_{i}=1(i=1, \ldots, N)$. Os resultados foram todos iguais a 4,516810841 , com a diferença estando sempre na décima casa decimal.

Para as simulações escolhemos o valor do parâmetro de convergência de Ewald $\alpha=3.5$. Essa escolha foi pautada no primeiro valor de $\alpha$ o qual fornecia $r_{\max }=1$, já que o somátório no espaço real é feito para toda varreduras, enquanto o espaço no espaço recíproco é feito apenas no início de cada simulação (vide seção A.3 para mais detalhes). 


\begin{tabular}{rcrrrr}
\hline \hline \multicolumn{5}{c}{$\mathrm{L}=16$} \\
\hline$\alpha$ & $r_{\max }$ & \multicolumn{1}{c}{$E_{\text {real }}$} & \multicolumn{1}{c}{$k_{\max }$} & \multicolumn{1}{c}{$E_{\text {recíproco }}$} & \multicolumn{1}{c}{$E_{\text {auto-interação }}$} \\
\hline 1.0 & 5 & 345,0872178219 & 26 & 907,5047132071 & $-96,2883555922$ \\
1.5 & 3 & 114,0276975136 & 40 & 1367,2490780471 & $-324,9732001235$ \\
2.0 & 2 & 23,7633008661 & 55 & 1902,8471193083 & $-770,3068447372$ \\
2.5 & 2 & 2,9993582817 & 69 & 2657,8097732821 & $-1504,5055561274$ \\
3.0 & 2 & 0,2252165777 & 84 & 3755,8639598462 & $-2599,7856009881$ \\
3.5 & 1 & 0,0100562628 & 98 & 5284,6567651866 & $-4128,3632460135$ \\
4.0 & 1 & 0,0002679535 & 112 & 7318,7580653804 & $-6162,4547578976$ \\
\hline \hline
\end{tabular}

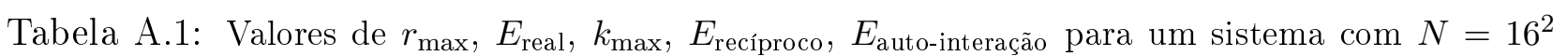
spins em função do parâmetro $\alpha$ para $\sigma_{i}=1(i=1, \ldots, N)$.

\begin{tabular}{ccrcrr}
\hline \hline & \multicolumn{4}{c}{$\mathrm{L}=32$} \\
\hline$\alpha$ & $r_{\max }$ & \multicolumn{1}{c}{$E_{\text {real }}$} & \multicolumn{1}{c}{$k_{\max }$} & \multicolumn{1}{c}{$E_{\text {recíproco }}$} & \multicolumn{1}{c}{$E_{\text {auto-interação }}$} \\
\hline 1.0 & 5 & 1380,3488712877 & 55 & 3630,0188528291 & $-385,1534223686$ \\
1.5 & 3 & 456,1107900542 & 82 & 5468,9963121876 & $-1299,8928004940$ \\
2.0 & 2 & 95,0532034645 & 109 & 7611,3884772332 & $-3081,2273789488$ \\
2.5 & 2 & 11,9974331266 & 136 & 10631,2390931159 & $-6018,0222245094$ \\
3.0 & 2 & 0,9008663107 & 163 & 15023,4558393576 & $-10399,1424039522$ \\
3.5 & 1 & 0,0402250511 & 190 & 21138,6270607830 & $-16513,4529840538$ \\
4.0 & 1 & 0,0010718140 & 217 & 29275,0322616094 & $-24649,8190315905$ \\
\hline \hline
\end{tabular}

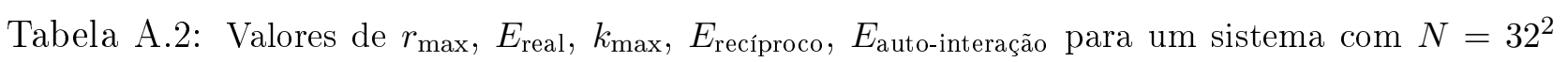
spins em função do parâmetro $\alpha$ para $\sigma_{i}=1(i=1, \ldots, N)$.

\begin{tabular}{ccrrrr}
\hline \hline & \multicolumn{4}{c}{$\mathrm{L}=48$} \\
\hline$\alpha$ & $r_{\max }$ & \multicolumn{1}{c}{$E_{\text {real }}$} & $k_{\max }$ & \multicolumn{1}{c}{$E_{\text {recíproco }}$} & \multicolumn{1}{c}{$E_{\text {auto-interação }}$} \\
\hline 1.0 & 5 & 3105,7849603959 & 110 & 8167,5424188166 & $-866,5952003294$ \\
1.5 & 3 & 1026,2492776226 & 157 & 12305,2417023946 & $-2924,7588011116$ \\
2.0 & 2 & 213,8697077951 & 200 & 17125,6240737505 & $-6932,7616026348$ \\
2.5 & 2 & 26,9942245349 & 244 & 23920,2879595186 & $-13540,5500051462$ \\
3.0 & 2 & 2,0269491990 & 287 & 33802,7756386665 & $-23398,0704088925$ \\
3.5 & 1 & 0,0905063649 & 330 & 47561,9108872513 & $-37155,2692141210$ \\
4.0 & 1 & 0,0024115815 & 373 & 65868,8225893010 & $-55462,0928210786$ \\
\hline \hline
\end{tabular}

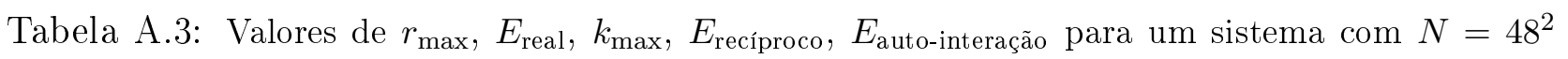
spins em função do parâmetro $\alpha$ para $\sigma_{i}=1(i=1, \ldots, N)$. 


\begin{tabular}{rrrrcr}
\hline \hline \multicolumn{5}{c}{$\mathrm{L}=56$} \\
\hline$\alpha$ & $r_{\max }$ & \multicolumn{1}{c}{$E_{\text {real }}$} & $k_{\max }$ & $E_{\text {recíproco }}$ & $E_{\text {auto-interação }}$ \\
\hline 1.0 & 5 & 4227,3184183162 & 98 & 11116,9327368750 & $-1179,5323560038$ \\
1.5 & 3 & 1396,8392945425 & 148 & 16748,8012061496 & $-3980,9217015130$ \\
2.0 & 2 & 291,1004356100 & 199 & 23309,8772115135 & $-9436,2588480307$ \\
2.5 & 2 & 36,7421389503 & 249 & 32558,1697226822 & $-18430,1930625600$ \\
3.0 & 2 & 2,7589030764 & 300 & 46009,3335079989 & $-31847,3736121037$ \\
3.5 & 1 & 0,1231892189 & 350 & 64737,0453733203 & $-50572,4497636647$ \\
4.0 & 1 & 0,0032824303 & 400 & 89654,7863008440 & $-75490,0707842459$ \\
\hline \hline
\end{tabular}

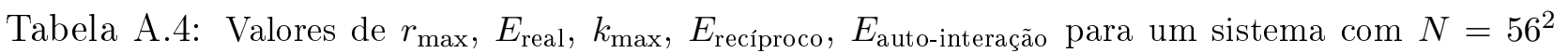
spins em função do parâmetro $\alpha$ para $\sigma_{i}=1(i=1, \ldots, N)$.

\begin{tabular}{ccrrrr}
\hline \hline & \multicolumn{3}{c}{$\mathrm{L}=72$} \\
\hline$\alpha$ & $r_{\max }$ & \multicolumn{1}{c}{$E_{\text {real }}$} & \multicolumn{1}{c}{$k_{\max }$} & \multicolumn{1}{c}{$E_{\text {recíproco }}$} & \multicolumn{1}{c}{$E_{\text {auto-interação }}$} \\
\hline 1.0 & 5 & 6988,0161608827 & 71 & 18376,8015263931 & $-1949,8392007411$ \\
1.5 & 3 & 2309,0608746530 & 136 & 27686,7937601842 & $-6580,7073025010$ \\
2.0 & 2 & 481,2068425395 & 201 & 38532,6541661106 & $-15598,7136059284$ \\
2.5 & 2 & 60,7370052036 & 265 & 53820,6479094247 & $-30466,2375115788$ \\
3.0 & 2 & 4,5606356977 & 330 & 76056,2451869313 & $-52645,6584200082$ \\
3.5 & 1 & 0,2036393210 & 395 & 107014,2994936860 & $-83599,3557317723$ \\
4.0 & 1 & 0,0054260583 & 460 & 148204,8508213100 & $-124789,7088474270$ \\
\hline \hline
\end{tabular}

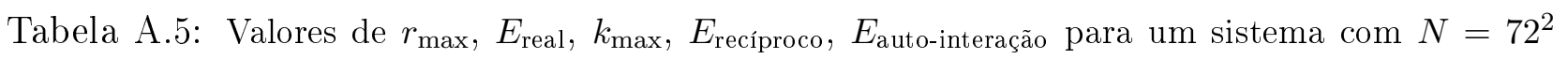
spins em função do parâmetro $\alpha$ para $\sigma_{i}=1(i=1, \ldots, N)$.

\section{A.5 Demonstrações das relações auxiliares}

\section{A.5.1 Demonstração da relação $§ 1$}

Nesta subseção apresentamos a demonstração da relação

$$
\sum_{|\vec{n}|=0}^{\infty} \delta\left(\vec{r}-\left(\vec{r}_{i j}+\vec{n}\right)\right)=\frac{1}{V_{d}} \sum_{|\vec{G}|=0}^{\infty} e^{i \vec{G} \cdot\left(\vec{r}-\vec{r}_{i j}\right)} .
$$

Apresentamos aqui uma expressão um pouco mais geral que a utilizada na seção sobre os aspectos analíticos do somatório de Ewald. Definimos um sistema em uma espaço com dimensão $d$ encerrado por uma caixa com lados iguais a $L_{1}, L_{2}, \ldots, L_{d}$, ou seja, com volume $V_{d}=\prod_{m=1}^{d} L_{m}$.

Nosso ponto de partida é a fórmula da soma de Poisson, que é dada por:

$$
\sum_{n=-\infty}^{\infty} f(x-n)=\sum_{k=-\infty}^{\infty} e^{2 \pi i k x} \int_{-\infty}^{\infty} f(\xi) e^{-2 \pi i k \xi} d \xi
$$


Substituindo a distribuição delta de Dirac $\delta(x)$ no lugar de $f(x)$ encontramos que

$$
\int_{-\infty}^{\infty} \delta(\xi) e^{-2 \pi i k \xi} d \xi=\left[e^{-2 \pi i k \xi}\right]_{\xi=0}=1
$$

e com isso

$$
\sum_{n=-\infty}^{\infty} \delta(x-n)=\sum_{k=-\infty}^{\infty} e^{2 \pi i k x}
$$

Sendo

$$
\vec{G}=2 \pi \sum_{m=1}^{d} \frac{k_{m}}{L_{m}} \hat{e}_{m}
$$

um vetor no espaço recíproco ${ }^{4}$, podemos reescrever o somatório do lado direito da equação (A.5.1) da seguinte maneira:

$$
\sum_{|\vec{G}|=0}^{\infty} e^{i \vec{G} \cdot\left(\vec{r}-\vec{r}_{i j}\right)}=\prod_{m=1}^{d} \sum_{k_{m}=-\infty}^{\infty} e^{2 \pi i k_{m} \hat{e}_{m} \cdot\left(\vec{r}-\vec{r}_{i j}\right) / L_{m}}
$$

Identificando $\hat{e}_{m} \cdot\left(\vec{r}-\vec{r}_{i j}\right) / L_{m}$ na expressão acima como $x_{m}$, a partir de (A.5.4), obtemos a igualdade:

$$
\sum_{|\vec{G}|=0}^{\infty} e^{i \vec{G} \cdot\left(\vec{r}-\vec{r}_{i j}\right)}=\prod_{m=1}^{d} \sum_{k_{m}=-\infty}^{\infty} \delta\left[\hat{e}_{m} \cdot\left(\vec{r}-\vec{r}_{i j}\right) / L_{m}-k_{m}\right]
$$

Sendo $\vec{n}=\sum_{m=1}^{d} L_{m} n_{m} \hat{e}_{m}$ (com $n_{m}$ inteiros positivos e negativos) temos $k_{m}=\hat{e}_{m} \cdot \vec{n} / L_{m}$. Substituindo $k_{m}$ na expressão acima obtemos:

$$
\prod_{m=1}^{d} \sum_{k_{m}=-\infty}^{\infty} \delta\left[\hat{e}_{m} \cdot\left(\vec{r}-\vec{r}_{i j}\right) / L_{m}-k_{m}\right]=\prod_{m=1}^{d} \sum_{n_{m}=-\infty}^{\infty} \delta\left[\hat{e}_{m} \cdot\left(\vec{r}-\vec{r}_{i j}-\vec{n}\right) / L_{m}\right]
$$

ou, na notação matricial:

$$
\sum_{|\vec{n}|=0}^{\infty} \delta\left[\mathbf{A}\left(\vec{r}-\vec{r}_{i j}-\vec{n}\right)\right]
$$

onde

$$
\mathbf{A}=\left(\begin{array}{cccc}
L_{1}^{-1} & 0 & \ldots & 0 \\
0 & L_{2}^{-1} & & 0 \\
\vdots & \vdots & \ddots & \vdots \\
0 & 0 & \ldots & L_{d}^{-1}
\end{array}\right)
$$

Utilizando a propriedade da delta de Dirac a seguir,

$$
\delta\left[\mathbf{A}\left(\vec{r}-\vec{r}_{i j}-\vec{n}\right)\right]=\frac{1}{|\operatorname{det} \mathbf{A}|} \delta\left(\vec{r}-\vec{r}_{i j}-\vec{n}\right),
$$

\footnotetext{
${ }^{4}$ Para uma explicação melhor sobre vetores no espaço recíproco vide capítulo 2 da Ref. [63].
} 
obtemos:

$$
\sum_{|\vec{G}|=0}^{\infty} e^{i \vec{G} \cdot\left(\vec{r}-\vec{r}_{i j}\right)}=V_{d} \sum_{|\vec{n}|=0}^{\infty} \delta\left(\vec{r}-\left(\vec{r}_{i j}+\vec{n}\right)\right)
$$

Portanto, como queríamos demonstrar

$$
\frac{1}{V_{d}} \sum_{|\vec{G}|=0}^{\infty} e^{i \vec{G} \cdot\left(\vec{r}-\vec{r}_{i j}\right)}=\sum_{|\vec{n}|=0}^{\infty} \delta\left(\vec{r}-\left(\vec{r}_{i j}+\vec{n}\right)\right) .
$$

Como mencionado, essa expressão é geral para $d$ dimensões com $V_{d}=L_{1}, \ldots, L_{d}$. Para um sistema bidimensional com lados iguais a $L$, teremos $V_{2}=L^{2}$ e esta expressão será idêntica a expressão utilizada na seção A.1 para obter $S_{\text {recíproco }}^{i j}$

\section{A.5.2 Demonstração da relação §2}

Nesta subseção apresentamos a demonstração da relação

$$
\int \frac{\gamma\left(\alpha^{2}|\vec{r}|^{2}\right)}{|\vec{r}|^{p}} e^{i \vec{G} \cdot \vec{r}} d^{2} \vec{r}=\pi \frac{G^{p-2}}{2^{p-2}} \Gamma\left(-\frac{p}{2}+1, \frac{G^{2}}{4 \alpha^{2}}\right),
$$

que é utilizada na seção para obter a parte recíproca do somatório de Ewald. Vale notar que esta é uma expressão específica para duas dimensões (isto é, $d=2$ ). Expressões para uma e três dimensões podem ser encontradas na Ref. [60].

Podemos explicitar os limites de integração do lado esquerdo da equação utilizando coordenadas polares:

$$
\int_{0}^{\infty} \int_{-\pi}^{\pi} \frac{\gamma\left(\frac{p}{2}, \alpha^{2} r^{2}\right)}{r^{p}} e^{i G r \cos (\theta)} r d r d \theta=\pi \frac{G^{p-2}}{2^{p-2}} \Gamma\left(-\frac{p}{2}+1, \frac{G^{2}}{4 \alpha^{2}}\right) .
$$

Utilizando a função de Bessel de ordem zero do primeiro tipo,

$$
J_{0}(G r)=\frac{1}{2 \pi} \int_{-\pi}^{\pi} e^{i G r \cos (\theta)} d \theta
$$

obtemos então a expressão (transformada de Hankel) que precisamos demostrar:

$$
2 \pi \int_{0}^{\infty} \frac{\gamma\left(\frac{p}{2}, \alpha^{2} r^{2}\right)}{r^{p-1}} J_{0}(G r) d r=\pi \frac{G^{p-2}}{2^{p-2}} \Gamma\left(-\frac{p}{2}+1, \frac{G^{2}}{4 \alpha^{2}}\right) .
$$

Fazendo a mudança de variável $u=G r$ e, em seguida, definido $I_{E}$ como o lado esquerdo e $I_{D}$ como o lado direito de (A.5.17):

$$
\begin{aligned}
& I_{E}(G)=2 \pi G^{p-2} \int_{0}^{\infty} \frac{\gamma\left(\frac{p}{2}, \frac{\alpha^{2} u^{2}}{G^{2}}\right)}{u^{p-1}} J_{0}(u) d u=G^{p-2} \tilde{I}_{E}(G) \\
& I_{D}(G)=\pi \frac{G^{p-2}}{2^{p-2}} \Gamma\left(-\frac{p}{2}+1, \frac{G^{2}}{4 \alpha^{2}}\right)=G^{p-2} \tilde{I}_{D}(G)
\end{aligned}
$$


reduz nosso propósito a mostrar que $\tilde{I}_{E}(G)=\tilde{I}_{D}(G)$. Podemos fazer isso mostrando que:

$$
\frac{d \tilde{I}_{E}}{d G}=\frac{d \tilde{I}_{D}}{d G}
$$

Ainda assim, $\tilde{I}_{E}(G)$ será igual a $\tilde{I}_{D}(G)$ a menos de uma constante. Só demonstramos de fato a igualdade se, ao impor valores no contorno, a igualdade ainda for observada.

Para o lado esquerdo teremos:

$$
\frac{d \tilde{I}_{E}}{d G}=2 \pi \int_{0}^{\infty} \frac{1}{u^{p-1}} \frac{d}{d G}\left[\gamma\left(\frac{p}{2}, \frac{\alpha^{2} u^{2}}{G^{2}}\right)\right] J_{0}(u) d u
$$

Fazendo $\frac{\alpha^{2} u^{2}}{G^{2}}=x$ obtemos:

$$
\frac{d}{d G}\left[\gamma\left(\frac{p}{2}, \frac{\alpha^{2} u^{2}}{G^{2}}\right)\right]=-\frac{2 \alpha^{2} u^{2}}{G^{3}} \frac{d}{d x}\left[\gamma\left(\frac{p}{2}, x\right)\right]
$$

Fazendo uso da relação (A.1.17) e retornando a variável original $G$, obtemos:

$$
\begin{aligned}
\frac{d}{d G}\left[\gamma\left(\frac{p}{2}, \frac{\alpha^{2} u^{2}}{G^{2}}\right)\right] & =-\frac{2 \alpha^{2} u^{2}}{G^{3}}\left[\frac{\alpha^{p-2} u^{p-2}}{G^{p-2}} e^{-\frac{\alpha^{2} u^{2}}{G^{2}}}\right] \\
& =-\frac{2 \alpha^{p} u^{p}}{G^{p+1}} e^{-\frac{\alpha^{2} u^{2}}{G^{2}}} .
\end{aligned}
$$

Substituindo (A.5.23) em (A.5.21) obtemos:

$$
\frac{d \tilde{I}_{E}}{d G}=-4 \pi \frac{\alpha^{p}}{G^{p+1}} \int_{0}^{\infty} u J_{0}(u) e^{-\frac{\alpha^{2} u^{2}}{G^{2}}} d u
$$

Na Ref. ([61]) temos a fórmula (6.631) para a integral:

$$
\int_{0}^{\infty} x^{\nu+1} e^{-\eta x^{2}} J_{\nu}(\beta x) d x=\frac{\beta^{\nu}}{(2 \eta)^{\nu+1}} e^{-\frac{\beta^{2}}{4 \eta}}
$$

$\operatorname{com}\{\eta \in \mathbf{R} \mid \eta>0\}$ e $\{\nu \in \mathbf{R} \mid \nu>-1\}$. Fazendo $\nu=0, \eta=\frac{\alpha^{2}}{G^{2}}, x=u$ e $\beta=1$ teremos:

$$
\int_{0}^{\infty} u J_{0}(u) e^{-\frac{\alpha^{2} u^{2}}{G^{2}}} d u=\frac{1}{2} \frac{G^{2}}{\alpha^{2}} e^{-\frac{G^{2}}{2 \alpha^{2}}} .
$$

Assim, finalmente obtemos:

$$
\frac{d \tilde{I}_{E}}{d G}=-4 \pi \frac{\alpha^{p}}{G^{p+1}} \frac{1}{2} \frac{G^{2}}{\alpha^{2}} e^{-\frac{G^{2}}{2 \alpha^{2}}}=-2 \pi \frac{\alpha^{p-2}}{G^{p-1}} e^{-\frac{G^{2}}{2 \alpha^{2}}}
$$

Fazendo então $\frac{G^{2}}{4 \alpha^{2}}=x$, obtemos a derivada de $\tilde{I}_{D}$ em relação a $G$ da seguinte maneira:

$$
\begin{aligned}
\frac{d \tilde{I}_{D}}{d G} & =\pi \frac{1}{2^{p-2}} \frac{d}{d G}\left[\Gamma\left(-\frac{p}{2}+1, \frac{G^{2}}{4 \alpha^{2}}\right)\right] \\
& =\pi \frac{1}{2^{p-2}} \frac{G}{2 \alpha^{2}} \frac{d}{d x}\left[\Gamma\left(-\frac{p}{2}+1, x\right)\right] \\
& =-\pi \frac{1}{2^{p-1}} x^{\left(-\frac{p}{2}+1\right)-1} e^{x} \frac{G}{\alpha^{2}}
\end{aligned}
$$


que escrito em função somente de $G$ fornece a expressão:

$$
\begin{aligned}
\frac{d \tilde{I}_{D}}{d G} & =-\pi \frac{1}{2^{p-1}}\left(\frac{G^{2}}{2^{2} \alpha^{2}}\right)^{-\frac{p}{2}} e^{-\frac{G^{2}}{4 \alpha^{2}}} \frac{G}{\alpha^{2}} \\
& =-2 \pi \frac{\alpha^{p-2}}{G^{p-1}} e^{-\frac{G^{2}}{4 \alpha^{2}}}
\end{aligned}
$$

Assim, como queríamos demonstrar, (A.5.27) é igual a (A.5.28). Portanto $\tilde{I}_{E}$ e $\tilde{I}_{D}$ são iguais a menos de uma constante.

\section{A.5.3 Demonstração da relação $\S 3$}

Nesta subseção apresentamos a demonstração da relação

$$
\sum_{|\vec{G}|=0}^{\infty} \mathcal{F}(G) e^{-i \vec{G} \cdot \vec{r}}=\sum_{|\vec{G}|=0}^{\infty} \mathcal{F}(G) \cos (\vec{G} \cdot \vec{r})
$$

Seja

$$
B(\vec{r})=\sum_{|\vec{G}|=0}^{\infty} \mathcal{F}(G) e^{-i \vec{G} \cdot \vec{r}}
$$

onde $\vec{G}=g_{1} \vec{e}_{1}+g_{2} \vec{e}_{2}$ com $g_{1}$ e $g_{2}$ números inteiros e $\vec{r}=r_{1} \vec{e}_{1}+r_{2} \vec{e}_{2}$ com $r_{1}$ e $r_{2}$ números reais. $\mathcal{F}(G)$ é uma função que depende somente do módulo do vetor $\vec{G}$, ou seja, de $G=\sqrt{g_{1}^{2}+g_{2}^{2}}$. Por simplicidade, vamos manter a notação $\mathcal{F}(G)$.

Explicitando os somatórios sobre $g_{1}$ e $g_{2}$ no somatório sobre o vetor $\vec{G}$ podemos reescrever $B(\vec{r})$ como

$$
B\left(r_{1}, r_{2}\right)=\sum_{g_{1}=-\infty}^{\infty} \sum_{g_{2}=-\infty}^{\infty} \mathcal{F}(G) e^{-i\left(g_{1} r_{1}+g_{2} r_{2}\right)}
$$

Podemos então separar o somatório sobre $g_{2}$ em dois intervalos: um para os valores negativos de $g_{2}$ e outro para os seus valores positivos, não esquecendo de incluir o termo para quando $g_{2}$ é zero. Assim obtemos

$$
B\left(r_{1}, r_{2}\right)=\sum_{g_{1}=-\infty}^{\infty}\left[\mathcal{F}(G) e^{-i g_{1} r_{1}}+\sum_{g_{2}=1}^{\infty} \mathcal{F}(G) e^{-i\left(g_{1} r_{1}+g_{2} r_{2}\right)}+\sum_{g_{2}=-\infty}^{-1} \mathcal{F}(G) e^{-i\left(g_{1} r_{1}+g_{2} r_{2}\right)}\right]
$$


De maneira semelhante, fazemos isso para o somatório sobre $g_{1}$ na expressão acima:

$$
\begin{array}{r}
B\left(r_{1}, r_{2}\right)=\mathcal{F}(0) \\
+\sum_{g_{1}=1}^{\infty} \mathcal{F}(G) e^{-i g_{1} r_{1}}+\sum_{g_{1}=-\infty}^{-1} \mathcal{F}(G) e^{-i g_{1} r_{1}} \\
+\sum_{g_{2}=1}^{\infty} \mathcal{F}(G) e^{-i g_{2} r_{2}}+\sum_{g_{2}=-\infty}^{-1} \mathcal{F}(G) e^{-i g_{2} r_{2}} \\
+\sum_{g_{1}=1}^{\infty} \sum_{g_{2}=1}^{\infty} \mathcal{F}(G) e^{-i\left(g_{1} r_{1}+g_{2} r_{2}\right)}+\sum_{g_{1}=-\infty}^{-1} \sum_{g_{2}=-\infty}^{-1} \mathcal{F}(G) e^{-i\left(g_{1} r_{1}+g_{2} r_{2}\right)} \\
+\sum_{g_{1}=1}^{\infty} \sum_{g_{2}=-\infty}^{-1} \mathcal{F}(G) e^{-i\left(g_{1} r_{1}+g_{2} r_{2}\right)}+\sum_{g_{1}=-\infty}^{-1} \sum_{g_{2}=1}^{\infty} \mathcal{F}(G) e^{-i\left(g_{1} r_{1}+g_{2} r_{2}\right)} .
\end{array}
$$

Podemos reescrever o primeiro termo de (A.5.32b) da seguinte maneira

$$
\sum_{g_{1}=1}^{\infty} \mathcal{F}(G) e^{-i g_{1} r_{1}}=\sum_{g_{1}=1}^{\infty} \mathcal{F}(G)\left[\cos \left(g_{1} r_{1}\right)-i \operatorname{sen}\left(g_{1} r_{1}\right)\right]
$$

Enquanto que para o segundo termo temos

$$
\sum_{g_{1}=-\infty}^{-1} \mathcal{F}(G) e^{-i g_{1} r_{1}}=\sum_{g_{1}=1}^{\infty} \mathcal{F}(G) e^{i g_{1} r_{1}}=\sum_{g_{1}=1}^{\infty} \mathcal{F}(G)\left[\cos \left(g_{1} r_{1}\right)+i \operatorname{sen}\left(g_{1} r_{1}\right)\right]
$$

Somando o primeiro e o segundo termo de (A.5.32b) teremos

$$
\begin{aligned}
& \sum_{g_{1}=1}^{\infty} \mathcal{F}(G) e^{-i g_{1} r_{1}}+\sum_{g_{1}=-\infty}^{-1} \mathcal{F}(G) e^{-i g_{1} r_{1}}= \\
= & \sum_{g_{1}=1}^{\infty} \mathcal{F}(G)\left[\cos \left(g_{1} r_{1}\right)-i \operatorname{sen}\left(g_{1} r_{1}\right)\right]+\sum_{g_{1}=1}^{\infty} \mathcal{F}(G)\left[\cos \left(g_{1} r_{1}\right)+i \operatorname{sen}\left(g_{1} r_{1}\right)\right] \\
= & 2 \sum_{g_{1}=1}^{\infty} \mathcal{F}(G) \cos \left(g_{1} r_{1}\right)=\sum_{g_{1}=1}^{\infty} \mathcal{F}(G) \cos \left(g_{1} r_{1}\right)+\sum_{g_{1}=-\infty}^{-1} \mathcal{F}(G) \cos \left(g_{1} r_{1}\right)
\end{aligned}
$$

Note que os termos do somatório que possuem a função seno se anulam e, devido à paridade da função cosseno, os limites dos somatórios em (A.5.33) podem ser escritos como os mesmos limites dos somatórios em (A.5.32b).

Para a soma dos somatórios em (A.5.32c) o resultado é análogo,

$$
\sum_{g_{2}=1}^{\infty} \mathcal{F}(G) e^{-i g_{2} r_{2}}+\sum_{g_{2}=-\infty}^{-1} \mathcal{F}(G) e^{-i g_{2} r_{2}}=\sum_{g_{2}=1}^{\infty} \mathcal{F}(G) \cos \left(g_{2} r_{2}\right)+\sum_{g_{2}=-\infty}^{-1} \mathcal{F}(G) \cos \left(g_{2} r_{2}\right)
$$


Olhando agora para a expressão (A.5.32d), podemos reescrever o primeiro termo como

$$
\begin{array}{r}
\sum_{g_{1}=1}^{\infty} \sum_{g_{2}=1}^{\infty} \mathcal{F}(G) e^{-i\left(g_{1} r_{1}+g_{2} r_{2}\right)}= \\
=\sum_{g_{1}=1}^{\infty} \sum_{g_{2}=1}^{\infty} \mathcal{F}(G)\left[\cos \left(g_{1} r_{1}+g_{2} r_{2}\right)-i \operatorname{sen}\left(g_{1} r_{1}+g_{2} r_{2}\right)\right],
\end{array}
$$

e o segundo termo como

$$
\begin{array}{r}
\sum_{g_{1}=-\infty}^{-1} \sum_{g_{2}=-\infty}^{-1} \mathcal{F}(G) e^{-i\left(g_{1} r_{1}+g_{2} r_{2}\right)}=\sum_{g_{1}=1}^{\infty} \sum_{g_{2}=1}^{\infty} \mathcal{F}(G) e^{i\left(g_{1} r_{1}+g_{2} r_{2}\right)} \\
=\sum_{g_{1}=1}^{\infty} \sum_{g_{2}=1}^{\infty} \mathcal{F}(G)\left[\cos \left(g_{1} r_{1}+g_{2} r_{2}\right)+i \operatorname{sen}\left(g_{1} r_{1}+g_{2} r_{2}\right)\right]
\end{array}
$$

Somando esses dois termos obtemos

$$
\begin{array}{r}
\sum_{g_{1}=1}^{\infty} \sum_{g_{2}=1}^{\infty} \mathcal{F}(G) e^{-i\left(g_{1} r_{1}+g_{2} r_{2}\right)}+\sum_{g_{1}=-\infty}^{-1} \sum_{g_{2}=-\infty}^{-1} \mathcal{F}(G) e^{-i\left(g_{1} r_{1}+g_{2} r_{2}\right)}= \\
=2 \sum_{g_{1}=1}^{\infty} \sum_{g_{2}=1}^{\infty} \mathcal{F}(G) \cos \left(g_{1} r_{1}+g_{2} r_{2}\right) \\
=\sum_{g_{1}=1}^{\infty} \sum_{g_{2}=1}^{\infty} \mathcal{F}(G) \cos \left(g_{1} r_{1}+g_{2} r_{2}\right)+\sum_{g_{1}=-\infty}^{-1} \sum_{g_{2}=-\infty}^{-1} \mathcal{F}(G) \cos \left(g_{1} r_{1}+g_{2} r_{2}\right) .
\end{array}
$$

Por último, temos que analisar (A.5.32e). Para o primeiro termo temos

$$
\begin{array}{r}
\sum_{g_{1}=1}^{\infty} \sum_{g_{2}=-\infty}^{-1} \mathcal{F}(G) e^{-i\left(g_{1} r_{1}+g_{2} r_{2}\right)}=\sum_{g_{1}=1}^{\infty} \sum_{g_{2}=1}^{\infty} \mathcal{F}(G) e^{-i\left(g_{1} r_{1}-g_{2} r_{2}\right)} \\
=\sum_{g_{1}=1}^{\infty} \sum_{g_{2}=1}^{\infty} \mathcal{F}(G)\left[\cos \left(g_{1} r_{1}-g_{2} r_{2}\right)-i \operatorname{sen}\left(g_{1} r_{1}-g_{2} r_{2}\right)\right]
\end{array}
$$

E para o segundo temos

$$
\begin{array}{r}
\sum_{g_{1}=-\infty}^{-1} \sum_{g_{2}=1}^{\infty} \mathcal{F}(G) e^{-i\left(g_{1} r_{1}+g_{2} r_{2}\right)}=\sum_{g_{1}=1}^{\infty} \sum_{g_{2}=1}^{\infty} \mathcal{F}(G) e^{i\left(g_{1} r_{1}-g_{2} r_{2}\right)} \\
=\sum_{g_{1}=1}^{\infty} \sum_{g_{2}=1}^{\infty} \mathcal{F}(G)\left[\cos \left(g_{1} r_{1}-g_{2} r_{2}\right)+i \operatorname{sen}\left(g_{1} r_{1}-g_{2} r_{2}\right)\right]
\end{array}
$$

Somando esses dois termos obtemos

$$
\begin{array}{r}
\sum_{g_{1}=1}^{\infty} \sum_{g_{2}=-\infty}^{-1} \mathcal{F}(G) e^{-i\left(g_{1} r_{1}+g_{2} r_{2}\right)}+\sum_{g_{1}=-\infty}^{-1} \sum_{g_{2}=1}^{\infty} \mathcal{F}(G) e^{-i\left(g_{1} r_{1}+g_{2} r_{2}\right)} \\
=2 \sum_{g_{1}=1}^{\infty} \sum_{g_{2}=1}^{\infty} \mathcal{F}(G) \cos \left(g_{1} r_{1}-g_{2} r_{2}\right)= \\
=\sum_{g_{1}=1}^{\infty} \sum_{g_{2}=-\infty}^{-1} \mathcal{F}(G) \cos \left(g_{1} r_{1}+g_{2} r_{2}\right)+\sum_{g_{1}=-\infty}^{-1} \sum_{g_{2}=1}^{\infty} \mathcal{F}(G) \cos \left(g_{1} r_{1}+g_{2} r_{2}\right) .
\end{array}
$$


Como $(\mathrm{A} .5 .32 \mathrm{~b})=(\mathrm{A} .5 .33),(\mathrm{A} .5 .32 \mathrm{c})=(\mathrm{A} .5 .34),(\mathrm{A} .5 .32 \mathrm{~d})=(\mathrm{A} .5 .35)$ e $(\mathrm{A} .5 .32 \mathrm{e})=(\mathrm{A} .5 .36)$, podemos reescrever $B\left(r_{1}, r_{2}\right)$ de acordo com as novas expressões obtidas, ou seja,

$$
\begin{array}{r}
B\left(r_{1}, r_{2}\right)=\mathcal{F}(0) \\
+\sum_{g_{1}=1}^{\infty} \mathcal{F}(G) \cos \left(g_{1} r_{1}\right)+\sum_{g_{1}=-\infty}^{-1} \mathcal{F}(G) \cos \left(g_{1} r_{1}\right) \\
+\sum_{g_{2}=1}^{\infty} \mathcal{F}(G) \cos \left(g_{2} r_{2}\right)+\sum_{g_{2}=-\infty}^{-1} \mathcal{F}(G) \cos \left(g_{2} r_{2}\right) \\
+\sum_{g_{1}=1}^{\infty} \sum_{g_{2}=1}^{\infty} \mathcal{F}(G) \cos \left(g_{1} r_{1}+g_{2} r_{2}\right)+\sum_{g_{1}=-\infty}^{-1} \sum_{g_{2}=-\infty}^{-1} \mathcal{F}(G) \cos \left(g_{1} r_{1}+g_{2} r_{2}\right) \\
+\sum_{g_{1}=1}^{\infty} \sum_{g_{2}=-\infty}^{-1} \mathcal{F}(G) \cos \left(g_{1} r_{1}+g_{2} r_{2}\right)+\sum_{g_{1}=-\infty}^{-1} \sum_{g_{2}=1}^{\infty} \mathcal{F}(G) \cos \left(g_{1} r_{1}+g_{2} r_{2}\right)
\end{array}
$$

Desde que cada somatório possui os mesmos limites apresentados em (A.5.32a) e que a cada exponencial esteja associada, como demonstrado, uma respectiva função cosseno, ou seja, as duas funções possuem a mesma dependência em $g_{1}$ e $g_{2}$, podemos "reconstruir" $B\left(r_{1}, r_{2}\right)$ em termos de um duplo somatório em $g_{1}$ e $g_{2}$ utilizando a função cosseno:

$$
B\left(r_{1}, r_{2}\right)=\sum_{g_{1}=-\infty}^{\infty} \sum_{g_{2}=-\infty}^{\infty} \mathcal{F}(G) \cos \left(g_{1} r_{1}+g_{2} r_{2}\right)
$$

Finalmente, retomando a notação vetorial obtemos

$$
B(\vec{r})=\sum_{|\vec{G}|=0}^{\infty} \mathcal{F}(G) \cos (\vec{G} \cdot \vec{r}),
$$

ou seja,

$$
\sum_{|\vec{G}|=0}^{\infty} \mathcal{F}(G) e^{-i \vec{G} \cdot \vec{r}}=\sum_{|\vec{G}|=0}^{\infty} \mathcal{F}(G) \cos (\vec{G} \cdot \vec{r})
$$




\section{Apêndice B}

\section{Informações técnicas}

Para a realização desse trabalho utilizamos quatro máquinas AMD Opteron 64bit com o sistema operacional Linux, distribuição Gentoo (release 2006.0). Duas máquinas eram constituídas de dois processadores 2,613 GHz Dual Core cada uma e as outras duas máguinas de dois processadores 2,412 GHz Dual Core cada uma, totalizando dezesseis processadores. Todos os códigos computacionais foram desenvolvidos na linguagem de programação fortran9o e o compilador utilizado foi o Intel@ Fortran.

A Tabela B mostra valores de tempo de CPU (em segundos) para cada um dos tamanhos de rede $L$. Esse tempo reflete o tempo de duração para realizar um único passo de Monte Carlo, ou seja, para realizar $L^{2}$ tentativas de atualização dos spins. As médias foram feitas sobre dezenas de séries com $10^{6}$ passos de Monte Carlo para $L=16,32$, 48 e de $5 \times 10^{5}$ passos de Monte Carlo para $L=56$ e 72 .

Escrevendo o tempo de CPU por meio de uma lei do tipo $\ln (t)=a+b \ln (L)$ obtemos uma estimativa grosseira de $a=-16,6(5)$ e $b=3,6(1)$, ou seja, temos

$$
t \sim L^{3,6}
$$

Vale ressaltar que os valores de tempo de CPU da Tabela B servem tanto para o algoritmo

\begin{tabular}{cc}
\hline \hline$L$ & $t(s)$ \\
\hline 16 & $1,6321(3) \times 10^{-3}$ \\
32 & $1,4895(2) \times 10^{-2}$ \\
48 & $7,621(1) \times 10^{-2}$ \\
56 & $1,3262(2) \times 10^{-1}$ \\
72 & $3,873(5) \times 10^{-1}$ \\
\hline \hline
\end{tabular}

Tabela B.1: Tempo necessário, em segundos, para cada um dos tamanhos de rede $L$, para realizar um único passo de Monte Carlo, ou seja, para realizar $L^{2}$ tentativas de atualização dos spins. 
de Metropolis quanto para o algoritmo multicanônico.

Outro detalhe importante é que, para a gerar números pseudo aleatórios nas simulações numéricas, nós utilizamos a subrotina ranmar proposta em [64]. 


\section{Referências Bibliográficas}

[1] FARROW, R. F. C. Molecular Beam Epitaxy: Applications to Key Materials. [S.1.]: Ed. Noyes Publications, Park Ridge, NJ, 1995.

[2] QIU, Z. Q.; BADER, S. D. Surface magneto-optic kerr effect. Rev. Sci. Instrum., AIP, v. 71, n. 3, p. 1243-1255, 2000. Disponível em: <http://link.aip.org/link/?RSI/71/1243/1>.

[3] PAPPAS, D. P.; KäMPER, K. P.; HOPSTER, H. Reversible transition between perpendicular and in-plane magnetization in ultrathin films. Phys. Rev. Lett., American Physical Society, v. 64, n. 26, p. 3179-3182, Jun 1990. Disponível em: $<$ http://prola.aps.org/abstract/PRL/v64/i26/p3179_1>.

[4] ALLENSPACH, R.; STAMPANONI, M.; BISCHOF, A. Magnetic domains in thin epitaxial co/au(111) films. Phys. Rev. Lett., American Physical Society, v. 65, n. 26, p. 3344-3347, Dez 1990. Disponível em: <http://prola.aps.org/abstract/PRL/v65/i26/p3344_1>.

[5] ALlENSPACH, R.; BISCHOF, A. Magnetization direction switching in fe/cu(100) epitaxial films: temperature and thickness dependence. Phys. Rev. Lett., American Physical Society, v. 69, n. 23, p. 3385-3388, Dez 1992. Disponível em: $<$ http://prola.aps.org/abstract/PRL/v69/i23/p3385_1>.

[6] PORTMAnN, O.; VATERLAUS, A.; PESCIA, D. An inverse transition of magnetic domain patterns in ultrathin films. Nature, Nature Publishing Group, v. 422, p. 701-704, Abr 2003. Disponível em: <http://dx.doi.org/10.1038/nature01538>.

[7] DE’BELl, K.; MACISAAC, A. B.; WHITEHEAD, J. P. Dipolar effects in magnetic thin films and quasi-two-dimensional systems. Rev. Mod. Phys., American Physical Society, v. 72, n. 1, p. 225-257, Jan 2000. Disponível em: $<$ http://link.aps.org/doi/10.1103/RevModPhys.72.225>.

[8] ISING, E. Beitrag zur theorie des ferromagnetismus. Zeitschrift für Physik, v. 31, 1925. 
[9] BRUSH, S. G. History of the lenz-ising model. Rev. Mod. Phys., American Physical Society, v. 39, n. 4, p. 883-893, Out 1967.

[10] SEUL, M.; ANDELMAN, D. Domain shapes and patterns: the phenomenology of modulated phases. Science, v. 267, n. 5197, p. 476-483, 1995. Disponível em: <http://www.sciencemag.org/cgi/content/abstract/267/5197/476>.

[11] FERNANDES, R. M. Propriedades físicas de sistemas com interações competitivas. 161 p. Tese (Doutorado) — Instituto de Física "Gleb Wataghin" da Universidade Estadual de Campinas, UNICAMP, 2008. Disponível em: $<$ http://webbif.ifi.unicamp.br/teses/apresentacao.php?filename=IF174>.

[12] BOOTH, I. et al. Domain structures in ultrathin magnetic films. Phys. Rev. Lett., American Physical Society, v. 75, n. 5, p. 950-953, Jul 1995. Disponível em: <http://link.aps.org/doi/10.1103/PhysRevLett.75.950>.

[13] SALINAS, S. R. A. Transições de fase e fenômenos críticos - Notas de aula. I Escola de Física da UFABC. Setembro 2008.

[14] STANLEY, H. E. Introduction to phase transitions and critical phenomena. [S.1.]: Claredon Press - Oxford, New York, 1987.

[15] BINDER, K. Theory of first-order phase transitions. Rep. Prog. Phys., v. 50, 1987.

[16] BINNEY, J. J. et al. The theory of critical phenomena. An introduction to the renormalization group theory. [S.1.]: Claredon Press - Oxford, New York, 1999.

[17] DECKER, K.; HASENFRATZ, A.; HASENFRATZ, P. Singular renormalization group transformations and first order phase transitions. Nucl. Phys. B, v. 295, 1988.

[18] FISHER, M. E.; BERKER, A. N. Scaling for first-order phase transitions in thermodynamic and finite systems. Phys. Rev. B, American Physical Society, v. 26, n. 5, p. 2507-2513, Set 1982. Disponível em: <http://link.aps.org/doi/10.1103/PhysRevB.26.2507>.

[19] LEE, J.; KOSTERLITZ, J. M. New method to study phase transitions. Phys. Rev. Lett., v. 65, 1990.

[20] LEE, J.; KOSTERLITZ, J. M. Tree-dimensional $q$-state potts model: Monte carlo study near $q=3$. Phys. Rev. B, v. 43, 1991. 
[21] PIGHIN, S. A.; CANNAS, S. A. Phase diagram of an ising model for ultrathin magnetic films: Comparing mean field and monte carlo predictions. Phys. Rev. B, American Physical Society, v. 75, n. 22, 2007. Disponível em: $<$ http://link.aps.org/abstract/PRB/v75/e224433>.

[22] IFTI, M. et al. A study of 2d ising ferromagnets with dipole interactions. Mod. Phys. Lett. B, World Scientific Publishing Company, v. 15, n. 21, p. 895-903, Set 2001.

[23] CASARTElli, M. et al. Metric features of a dipolar model. J. Phys. A: Math. Gen., IOP Publishing Limited, v. 37, p. 11731-11749, Nov 2004. Disponível em: <http://dx.doi.org/10.1088/0305-4470/37/49/001>.

[24] MACISAAC, A. B. et al. Striped phases in two-dimensional dipolar ferromagnets. Phys. Rev. B, American Physical Society, v. 51, n. 22, p. 16033-16045, Jun 1995. Disponível em: $<$ http://prola.aps.org/abstract/PRB/v51/i22/p16033_1>.

[25] BISKUP, M.; CHAYES, L.; KIVELSON, S. On the absence of ferromagnetism in typical 2d ferromagnets. Commun. Math. Phys., Springer Berlin / Heidelberg, v. 274, p. 217-231, Ago 2007. Disponível em: <http://dx.doi.org/10.1007/s00220-007-0260-0>.

[26] GIUliani, A.; LEBOWITZ, J. L.; LIEB, E. H. Ising models with longrange antiferromagnetic and short-range ferromagnetic interactions. Phys. Rev. B, American Physical Society, v. 74, n. 6, p. 064420, 2006. Disponível em: $<$ http://link.aps.org/abstract/PRB/v74/e064420>.

[27] GIUliAnI, A.; LEBOWITZ, J. L.; LIEB, E. H. Striped phases in two-dimensional dipole systems. Phys. Rev. B, American Physical Society, v. 76, n. 18, p. 184426, 2007. Disponível em: <http://link.aps.org/abstract/PRB/v76/e184426>.

[28] GAREL, T.; DONIACH, S. Phase transitions with spontaneous modulation-the dipolar ising ferromagnet. Phys. Rev. B, American Physical Society, v. 26, n. 1, p. 325-329, Jul 1982. Disponível em: <http://link.aps.org/doi/10.1103/PhysRevB.26.325>.

[29] KASHUBA, A. B.; POKROVSKY, V. L. Stripe domain structures in a thin ferromagnetic film. Phys. Rev. B, American Physical Society, v. 48, n. 14, p. 10335-10344, Out 1993. Disponível em: <http://prola.aps.org/abstract/PRB/v48/i14/p10335_1>. 
[30] ABANOV, A. et al. Phase diagram of ultrathin ferromagnetic films with perpendicular anisotropy. Phys. Rev. B, American Physical Society, v. 51, n. 2, p. 1023-1038, Jan 1995. Disponível em: <http://link.aps.org/doi/10.1103/PhysRevB.51.1023>.

[31] HURLEY, M. M.; SINGER, S. J. Domain-array melting in the dipolar lattice gas. Phys. Rev. B, American Physical Society, v. 46, n. 9, p. 5783-5786, Sep 1992. Disponível em: $<$ http://prola.aps.org/abstract/PRB/v46/i9/p5783_1>.

[32] RASTElli, E.; REgINA, S.; TASSI, A. Phase diagram of a square ising model with exchange and dipole interactions: Monte carlo simulations. Phys. Rev. B, American Physical Society, v. 76, n. 5, p. 054438, 2007. Disponível em: $<$ http://link.aps.org/abstract/PRB/v76/e054438>.

[33] CANNAS, S. A. et al. Ising nematic phase in ultrathin magnetic films: A monte carlo study. Phys. Rev. B, American Physical Society, v. 73, p. 184425-184437, Mai 2006. Disponível em: <http://link.aps.org/doi/10.1103/PhysRevB.73.184425>.

[34] CHAIKIN, P. M.; LUBENSKY, T. C. Principles of condensed matter physics. [S.1.]: Cambridge University Press. Cambridge, New York, 1995.

[35] CANNAS, S. A.; STARIOLO, D. A.; TAMARIT, F. A. Stripe-tetragonal first-order phase transition in ultrathin magnetic films. Phys. Rev. B, American Physical Society, v. 69, n. 9, p. 092409, Mar 2004. Disponível em: $<$ http://link.aps.org/doi/10.1103/PhysRevB.69.092409>.

[36] RASTELli, E.; REGINA, S.; TASSI, A. Phase transitions in a square ising model with exchange and dipole interactions. Phys. Rev. B, American Physical Society, v. 73, p. 144418-144429, Abr 2006. Disponível em: $<$ http://link.aps.org/doi/10.1103/PhysRevB.73.144418>.

[37] ARLETT, J. et al. Phase diagram for the striped phase in the two-dimensional dipolar ising model. Phys. Rev. B, American Physical Society, v. 54, n. 5, p. 3394-3402, Ago 1996. Disponível em: < http://prola.aps.org/abstract/PRB/v54/i5/p3394_1>.

[38] HUANG, K. Statistical Mechanics. [S.1.]: Wiley; 2nd edition, 1987.

[39] BAnHot, G. The metropolis algorithm. Rep. Prog. Phys., v. 51, 1988. Disponível em: $<$ http://www.iop.org/EJ/article/0034-4885/51/3/003/rpv51i3p429.pdf>. 
[40] METROPOLIS, N. et al. Equation of state calculations by fast computing machines. J. Chem. Phys., AIP, v. 21, n. 6, p. 1087-1092, Jun 1953. Disponível em: $<$ http://link.aip.org/link/?JCP/21/1087/1>.

[41] SOKAL, A. D. Monte Carlo methods in statistical mechanics: foundations and new algorithms. Lecture notes of the cours de troisime cycle de la physique en suisse romande. [S.1.]: Lausanne, 1989.

[42] BERG, B. A. Introduction to multicanonical monte carlo simulations. Fields Inst. Commun., v. 26, p. 1, 2000. Disponível em: <http://www.citebase.org/abstract?id=oai:arXiv.org:cond-mat/9909236>.

[43] BERG, B. A.; NEUHAUS, T. Multicanonical ensemble: a new approach to simulate firstorder phase transitions. Phys. Rev. Lett., American Physical Society, v. 68, n. 1, p. 9, Jan 1992.

[44] BERG, B. A.; NEUHAUS, T. Multicanonical algorithms for first order phase transitions. Phys. Lett. B, v. 267, p. 249-253, Set 1991. Disponível em: $<$ http://dx.doi.org/10.1016/0370-2693(91)91256-U>.

[45] BERG, B. A. Multicanonical simulations step by step. Comp. Phys. Commun., v. 153, n. 3, p. 397-406, 2003. Disponível em: <http://dx.doi.org/10.1016/S0010-4655(03)00245$5>$.

[46] WANG, F.; LANDAU, D. P. Efficient, multiple-range random walk algorithm to calculate the density of states. Phys. Rev. Lett., American Physical Society, v. 86, n. 10, p. 2050-2053, Mar 2001.

[47] LEE, J. New monte carlo algorithm: entropic sampling. Phys. Rev. Lett., American Physical Society, v. 71, n. 2, p. 211-214, Jul 1993.

[48] Allen, M. P.; TILDESLEY, D. J. Computer Simulation of Liquids. [S.1.]: Claredon Press - Oxford, 1989.

[49] BINDER, K.; HEERMAN, D. W. Monte Carlo Simulation in Statistical Physics, An Introduction. [S.1.]: Springer Series in Solid-State Sciences 80, 1987.

[50] ALVES, N. A.; BERG, B. A.; SANIELEVICI, S. Spectral density study of the su(3) deconfining phase transition. Nucl. Phys. B, Elsevier B. V., v. 376, n. 1, p. 218-252, Jun 1992. 
[51] FERRENBERG, A. M.; SWENDSEN, R. H. New monte carlo technique for studying phase transitions. Phys. Rev. Lett., American Physical Society, v. 61, n. 23, p. 2635-2638, Dez 1988. Disponível em: <http://link.aps.org/doi/10.1103/PhysRevLett.61.2635>.

[52] FERRENBERG, A. M.; SWENDSEN, R. H. Optimized monte carlo data analysis. Phys. Rev. Lett., American Physical Society, v. 63, n. 12, p. 1195-1198, Set 1989. Disponível em: $<$ http://link.aps.org/doi/10.1103/PhysRevLett.63.1195>.

[53] BERG, B. A. Markov Chain Monte Carlo Simulations And Their Statistical Analysis: With Web-based Fortran Code. [S.1.]: World Scientific Publishing Co. Pte. Ltd., 2004.

[54] OSSOLA, G.; SOKAL, A. D. Dynamic critical behavior of the swendsen-wang algorithm for the three-dimensional ising model. Nucl. Phys. B, v. 691, p. 259-291, 2004.

[55] CANNAS, S. A. et al. Interplay between coarsening and nucleation in an ising model with dipolar interactions. Phys. Rev. E, American Physical Society, v. 78, p. 051602, 2008. Disponível em: <http://link.aps.org/doi/10.1103/PhysRevE.78.051602>.

[56] EWALD, P. P. Die berechnung optischer und elektrostatischer gitterpotentiale. Annalen der Physik, v. 369, p. 253-287, Ago 1921. Disponível em: <http://www3.interscience.wiley.com/journal/112485052/abstract>.

[57] TOUKMAJI, A. Y.; JR., J. A. B. Ewald summation techniques in perspective: a survey. Comp. Phys. Comm., Elsevier B. V., v. 95, p. 73-92, Jun 1996. Disponível em: <http://dx.doi.org/10.1016/0010-4655(96)00016-1>.

[58] WIDMANN, A. H.; ADOLF, D. B. A comparison of ewald summation techniques for planar surfaces. Comp. Phys. Comm., Elsevier B. V., v. 107, p. 167-186, 1997. Disponível em: <http://dx.doi.org/10.1016/S0010-4655(97)00090-8>.

[59] GAO, G. T.; ZENG, X. C.; WANG, W. Vapor-liquid coexistence of quasi-two-dimensional stockmayer fluids. J. Chem. Phys., AIP, v. 106, n. 8, p. 3311-3317, Fev 1997. Disponível em: <http://link.aip.org/link/?JCP/106/3311/1>.

[60] NIJBOER, B. R. A.; WETTE, F. W. de. On the calculation of lattice sums. Physica, Elsevier B. V., v. 23, p. 309-321, Jan 1957. Disponível em: <http://dx.doi.org/10.1016/S00318914(57)92124-9>.

[61] GRADSHTEYN, I. S.; RHYZIK, I. M. Tables of Integrals, Series and Products. [S.l.]: Academic - New York, 1980. 
[62] DANESE, G. et al. Ewald potentials evaluated through look-up tables. Comp. Phys. Commun., Elsevier B. V., v. 108, p. 211-217, 1998. Disponível em: $<$ http://dx.doi.org/10.1016/S0010-4655(97)00140-9>.

[63] KITTEL, C. Introduction to Solid State Physics. [S.l.]: John Wiley and Sons, Inc - New York, 1996.

[64] MARSAGLIA, G.; ZAMAN, A.; TSANG, W. W. Toward a universal random number generator. Stat. Prob. Lett., v. 9, 1990. 